Aus der Abteilung Mund-, Kiefer- und Gesichtschirurgie

(Prof. Dr. med. Dr. med. dent. H. Schliephake)

im Zentrum Zahn-, Mund- und Kieferheilkunde

der Medizinischen Fakultät der Georg-August-Universität Göttingen

\title{
Darstellbarkeit von Knochenstrukturen und enossalen Implantaten mit der digitalen Volumentomographie und zweidimensionalem Zahnfilmverfahren
}

\author{
INAUGURAL - DISSERTATION \\ zur Erlangung des Doktorgrades \\ für Zahnheilkunde \\ der Medizinischen Fakultät der \\ Georg-August-Universität zu Göttingen
}

vorgelegt von

Isabel Steinbacher

aus

Cuxhaven

Göttingen, 2016 
Dekan:

1. Berichterstatter/in:

2. Berichterstatter/in:

3. Berichterstatter/in:
Prof. Dr. rer. nat. H. K. Kroemer PD Dr. med. dent. S. Sennhenn-Kirchner Prof. Dr. Christina Unterberg-Buchwald Tag der mündlichen Prüfung: $\quad$ 25.10.2016 


\section{$\underline{\text { Inhaltsverzeichnis }}$}

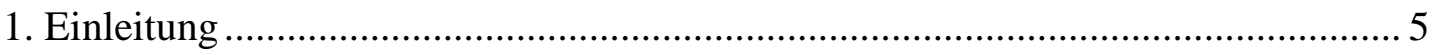

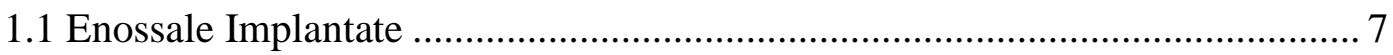

1.2 Implantologische Behandlungsplanung und Diagnostik ................................ 10

1.3 Zahnärztliche Röntgentechniken............................................................ 12

1.3.1 Zweidimensionale Röntgenverfahren .................................................. 12

1.3.1.1 Konventionelle Zahnfilmaufnahmen ................................................. 12

1.3.1.2 Digitale Zahnfilmaufnahmen ........................................................... 13

1.3.2 Dreidimensionale Röntgenverfahren .................................................. 15

1.3.2.1 Digitale Volumentomographie (DVT) .............................................. 15

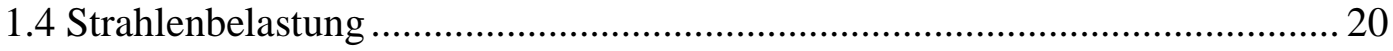

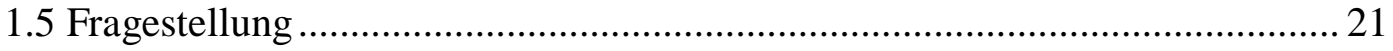

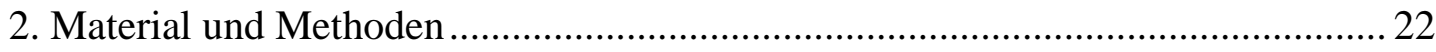

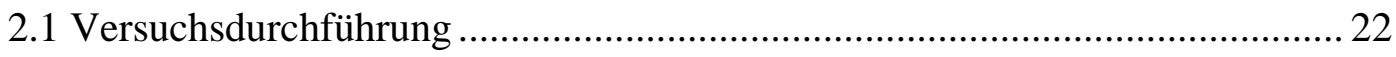

2.1.1 Überblick über die Versuchsdurchführung ........................................... 22

2.1.2 Flussdiagramm der Versuchsdurchführung ......................................... 23

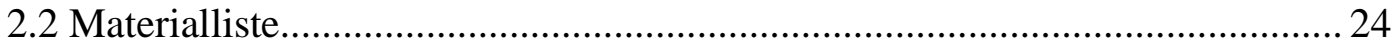

2.3. Schweinekiefermodelle mit enossalen Implantaten ..................................... 25

2.4 Defekte an Implantaten, Alveolarknochen und Fremdkörper.......................... 26

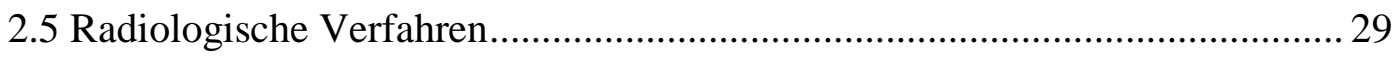

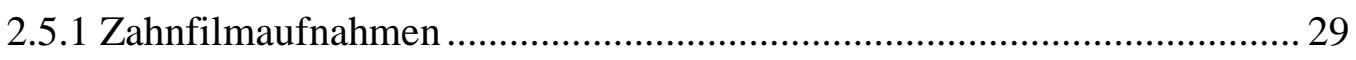

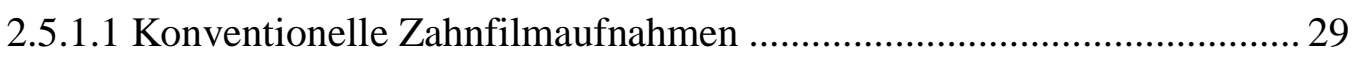

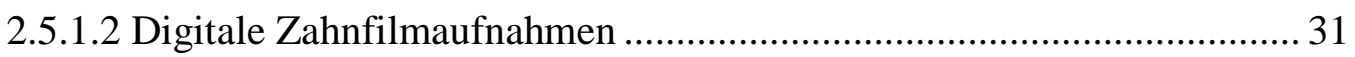

2.5.2 Digitale Volumentomographie (DVT) ................................................. 34

2.6 Randomisierte Evaluation durch zehn Untersucher ...................................... 37

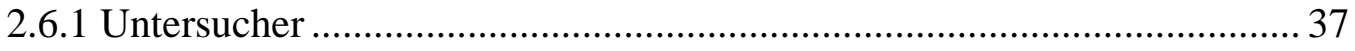

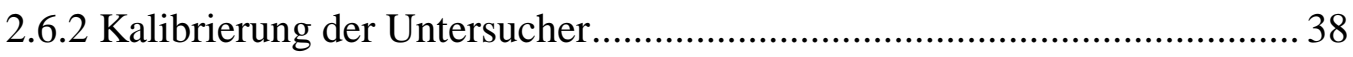

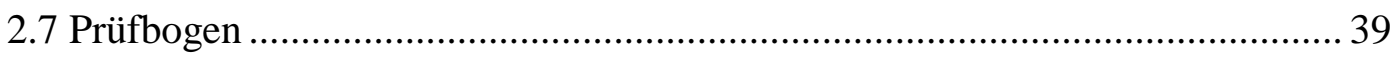

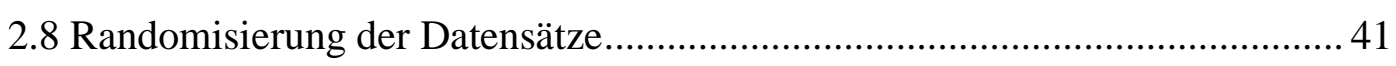

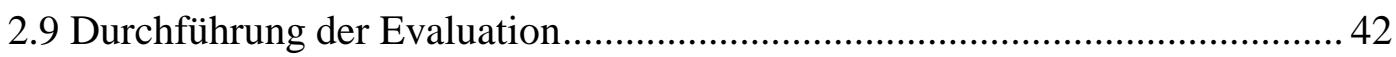




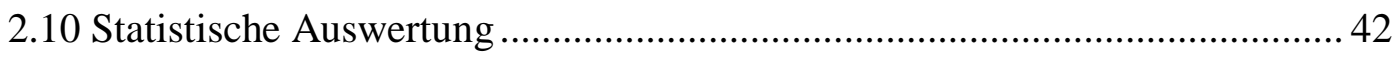

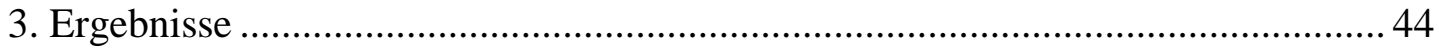

3.1 Bildbeispiele zur Darstellbarkeit in den unterschiedlichen............................ 44

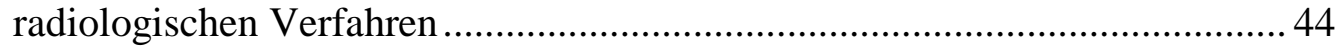

3.2 Der Vergleich der radiologischen Verfahren ............................................. 50

3.2.1 Differenzierung der Nachbargewebe .................................................. 50

3.2.2 Längenmessung der Implantate........................................................ 50

3.2.3 Durchmesserbestimmung der Implantate............................................ 53

3.2.4 Evaluation der iatrogen gesetzten Alveolar- und Implantatdefekte.......... 57

3.2.5 Beeinträchtigung der Bildauswertung durch Aufhärtungsartefakte.......... 77

3.3 Zweidimensionale versus dreidimensionale Verfahren ................................. 78

3.4. Vergleich der Untersucher ................................................................. 83

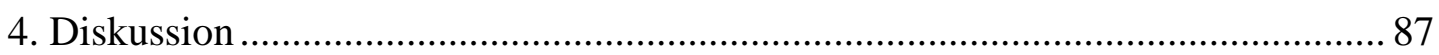

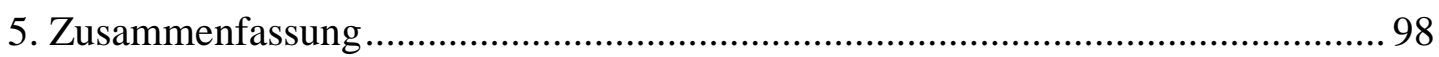

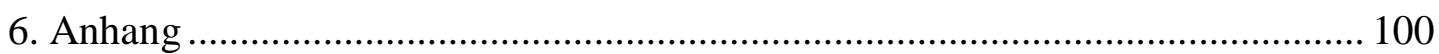

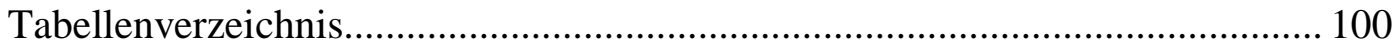

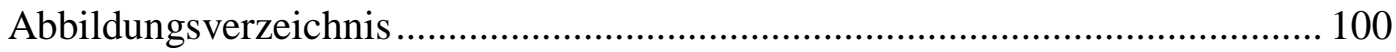

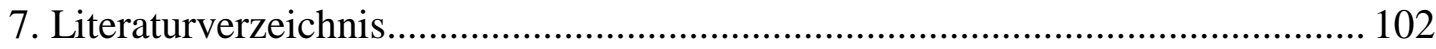




\section{Abkürzungsverzeichnis}

\begin{tabular}{|c|c|}
\hline$\mu \mathrm{m}$ & Mikrometer \\
\hline$\mu \mathrm{Sv}$ & Mikrosievert \\
\hline $3 \mathrm{D}$ & dreidimensional \\
\hline Abb. & Abbildung \\
\hline ALARA & as low as reasonably achievable \\
\hline bzw. & beziehungsweise \\
\hline $\mathrm{CAD} / \mathrm{CAM}$ & computer aided design / computer aided manufacturing \\
\hline CCD-Sensor & charged-coupled device-Sensor \\
\hline $\mathrm{cm}$ & Zentimeter \\
\hline CMOS & complementary metal-oxide-semiconductor \\
\hline CT & Computertomographie \\
\hline CTDI & computed tomography dose index \\
\hline dig. ZF & digitaler Zahnfilm \\
\hline DVT & digitale Volumentomographie \\
\hline FOV & field of view \\
\hline FPD & flatpanel detector \\
\hline HA & Hydroxylapatit \\
\hline $\mathrm{HU}$ & hounsfield units \\
\hline IID & image intensifier detector \\
\hline konv. ZF & konventioneller Zahnfilm \\
\hline $\mathrm{kV}$ & Kilovolt \\
\hline $\mathrm{mA}$ & Milliampere \\
\hline MAR & Metallartefaktreduktion \\
\hline $\mathrm{mm}$ & Millimeter \\
\hline $\min$. & mindestens \\
\hline MSCT & multislice computed tomography \\
\hline$\varnothing$ & Durchmesser \\
\hline OPG & Orthopantomogramm \\
\hline PSP & photo-stimulable phosphor \\
\hline Sek. & Sekunden \\
\hline ß-ТСР & beta-Tricalciumphosphat-Keramik \\
\hline Stdf. & Standardfehler \\
\hline Tab. & Tabelle \\
\hline PS & titan-plasma-flamespray \\
\hline z. B. & zum Beispiel \\
\hline
\end{tabular}




\section{Einleitung}

Die Implantologie hat sich in den letzten zwei Jahrzehnten zu einem wichtigen Feld in der Zahnheilkunde entwickelt. Dabei wurden Behandlungsmethoden, Materialien und Form der Implantate ständig verändert und weiter untersucht. Die Popularität wuchs im klinischen Alltag deutlich an. Heute besitzt die Implantologie eine hohe Akzeptanz und Nachfrage bei Patienten hinsichtlich des Ersatzes einzelner Zähne oder zum Ermöglichen eines festsitzenden Zahnersatzes bei zahnlosem Kiefer (Mupparapu und Singer 2004). Dieser Fachbereich hat sich von der erfolgreichen Implantation mit der grundlegenden Osseointegration des Implantates in den Kieferknochen bis hin zu hochästhetischen Lösungen eines festsitzenden Zahnersatzes entwickelt (Hämmerle et al. 2004). Die natürliche Kaufunktion wird wiederhergestellt und die Kontur sowie der Gingivaverlauf werden einem natürlichen Zahn nachempfunden (Klokkevold und Newman 2000; Jivraj und Chee 2006).

Heute werden enossale Implantate mit zahnwurzelähnlicher, zylindrischer Form verwendet. Sie haben sich im Laufe der Jahre gegen die subperiostalen, submukösen und transossalen Implantatsysteme durchgesetzt. Seit den Untersuchungen von Brånemark im Jahre 1977 zur Osseointegration von Titanimplantaten in den Knochen wird dieses Material größtenteils zur Implantation in der Zahnmedizin verwendet (Binon 2000).

Die Oberfläche der Titanimplantate wurde in Hinblick auf die Oberflächenbenetzbarkeit und die folgende Osseointegration untersucht und verändert. Es entstanden unterschiedliche Techniken zur Oberflächenbearbeitung wie das Maschinieren, die Beschichtung oder das Aufrauen von Implantaten. Die Beschichtung der Implantate kann zum Beispiel aus titanplasma-flamespray (TPS), Hydroxylapatit oder strukturierten Oxiden bestehen. Die Oberflächenrauigkeit wird durch Säureätzung, Sandstrahlung oder eine Kombination dieser beiden Techniken erreicht (Brunski et al. 2000).

Eine Weiterentwicklung des Implantatdesigns sollte einen positiven Effekt auf einen besseren Implantat-Knochen-Kontakt haben und damit die Einheilung verbessern. 
Die erfolgreiche Einheilung eines Implantates hängt zunächst von der Primärstabilität ab, die für die folgende Sekundärstabilität und die Osseointegration entscheidend ist. Die Primärstabilität entsteht durch die Übereinstimmung des operativ hergestellten Implantatbettes im Knochen und das darin inserierte Implantat. Sie ist abhängig vom makroskopischen Design des Implantates, der Knochendichte und der operativen Vorgehensweise (Sennerby und Meredith 2008; Rodrigo et al. 2010).

Die Sekundärstabilität ist definiert als der schrittweise Umbau und Zuwachs von Knochengewebe zwischen Implantat und Knochen und ist schließlich mit dem vollständigen Implantat-Knochen-Kontakt abgeschlossen, wenn der Raum zwischen den Implantatwindungen mit Knochengewebe gefüllt und das Implantat osseointegriert ist (Rodrigo et al. 2010).

Die Osseointegration eines Implantates benötigt eine belastungsfreie Einheilungsphase von drei bis sechs Monaten. Kommt es innerhalb dieser Phase zur Belastung des Implantates, kann eine fibro-ossäre Einheilung die Folge sein. Dabei wird das Implantat von narbigem Ersatzgewebe umschlossen und eine solide Einheilung in den Knochen bleibt aus (Albrektsson et al. 1981). Die Stabilität und Osseointegration des Implantates in den Knochen stellt den klinischen Behandlungserfolg nach Implantation dar (Sennerby und Meredith 2008).

Dieser Behandlungserfolg setzt unter anderem ein ausreichendes Knochenangebot und eine gute Knochenqualität voraus. In der präoperativen Behandlungsphase sollte daher zunächst die entsprechende Diagnostik durchgeführt werden. Die Röntgendiagnostik ist eines der entscheidenden Werkzeuge zur Beurteilung des vorhandenen Knochenangebotes und der umliegenden, angrenzenden Strukturen wie des Canalis mandibulae und des Sinus maxillaris (Mupparapu und Singer 2004).

In der Basisuntersuchung wird dabei zunächst ein Orthopantomogramm (OPG) als Übersichtsaufnahme erstellt. Befundbezogene Aufnahmen können zum Beispiel in Form einer dreidimensionalen Aufnahme (DVT oder CT) folgen (Nitsche et al. 2012). Im weiteren Verlauf der Behandlung wird ein postoperatives Röntgenbild zur Überprüfung der Implantatposition erstellt sowie in regelmäßigen Verlaufskontrollen das Knochenniveau um das Implantat verglichen (DelBalso et al. 1994). 
Mit der steigenden Anzahl der inserierten Implantate und dem höheren Anspruch an die Implantatversorgungen haben sich in den letzten Jahrzehnten auch die Erwartungen Diagnostik und Nachsorge intensiviert.

In den letzten Jahren hat sich die digitale Volumentomographie (DVT) als zahnärztliches dreidimensionales Röntgenverfahren etabliert (Scarfe 2006).

Es zeichnet sich durch eine hochauflösende Darstellung von Hartgeweben, eine isometrische Auflösung und ein dreidimensionales Nutzstrahlenfeld aus (Schulze et al. 2009). Verglichen mit der Computertomographie (CT) kann bei einer DVT-Aufnahme das Aufnahmevolumen an die diagnostischen Anforderungen angepasst werden. Dies führt zu einer verringerten Aufnahmezeit und einer reduzierten Strahlenbelastung (Mohan et al. 2011; Howerton und Mora 2008). Des Weiteren kann ein DVT-Gerät in einer zahnärztlichen Praxis mit einer entsprechenden Röntgenfachkunde direkt durch einen Zahnarzt betrieben werden (Patel 2009; Nitsche et al. 2012).

\subsection{Enossale Implantate}

Die ersten dentalen Implantate wurden in den 1940er Jahren von Adams entwickelt. Die zunächst enossalen Implantate wurden später durch Dahl in Schweden zu subperiostal gelagerten Implantaten verändert. In den 1950er Jahren veränderte sich die Positionierung der Implantate hin zum größten Knochenangebot im Kiefer.

Dr. Linkow entwickelte Mitte der 1960er Jahre das Blattimplantat für Ober- und Unterkiefer. 1977 wurde durch Brånemark ein bedeutender Grundstein für die heutige Implantologie gelegt. Er entdeckte, dass zwischen Knochen und Implantat nach einer bestimmten Zeitspanne eine feste Verbindung entsteht und dass das Implantat in den Knochen einheilt (Abraham 2014). Brånemarks Titanimplantat mit einem Doppelgewinde in zylindrischer Form hat sich heute durchgesetzt. Unter den Herstellern variieren gegenwärtig Formunterschiede und Oberflächenveränderungen, die der verbesserten Osseointegration dienen sollen (Binon 2000). Der Einsatz von Implantaten zum Ersetzen einzelner Zähne oder zur Befestigung von herausnehmbarem Zahnersatz auf dem zahnlosen Kiefer ist heute fester Bestandteil der täglichen Praxis. Die Voraussetzung zur erfolgreichen Behandlung ist ein ausreichendes Knochenangebot sowie eine entsprechende Knochenqualität, um die Einheilung -die 
Osseointegration- des Implantates in den Knochen zu sichern (Sennerby und Meredith 2008; Hiasa et al. 2011).

Nach Insertion des Implantates ist die Primärstabilität entscheidend für die weitere Einheilung des Implantates. Das Implantat und das im Knochen präparierte Implantatbett zeigen dabei möglichst geringe Divergenz, so dass das Implantat sicher im Knochen verankert und damit das Risiko von Mikrobewegungen gering ist (Rodrigo et al. 2010). Die primäre Stabilität ist dabei von dem Knochen, der operativen Technik und der verwendeten Implantatform abhängig (Sennerby und Meredith 2008). Um die Einheilung des Implantates zu erreichen, wird dieses nach Insertion für eine Einheilungsphase von drei bis sechs Monaten belastungsfrei im Kiefer belassen. In dieser Phase kann die Knochenapposition an der Implantatoberfläche störungsfrei ablaufen (Albrektsson et al. 1981).

Heute unterscheidet man zwischen verschiedenen Zeitpunkten zur Implantation:

Die Sofortimplantation nach vorheriger Extraktion des Zahnes, die frühe Implantation mit Weichgewebsheilung (4-8 Wochen post extractionem), die frühe Implantation mit partieller Knochenheilung (3-4 Monate post extractionem) und die späte Implantation (mehr als 4 Monate post extrationem). Durch die sofortige oder frühe Implantation wird die Behandlungszeit verkürzt, vor allem im ästhetischen Bereich kann eine Sofortimplantation mit einer umgehenden Belastung des Implantates kombiniert werden (Hämmerle et al. 2004). Die unbelastete Einheilung nach Sofortimplantation variiert dabei stark zwischen sieben Tagen und zwei Monaten (Schliephake et al. 2012). Eine Sofortimplantation sollte nur dann durchgeführt werden, wenn nach Extraktion eine Primärstabilität des Implantates durch die Form des verbleibenden Alveolarkammes sichergestellt ist. Bei einer Defektdimension um das Implantat von $2 \mathrm{~mm}$ oder weniger kann von einer spontanen Einheilung mit Osseointegration des Implantates ausgegangen werden. Ist der Defekt in der Alveole größer als $2 \mathrm{~mm}$, sollte eine zusätzliche Augmentation stattfinden, sowohl um die Primärstabilität zu sichern als auch das Volumen des umgebenden Knochens zu erhalten (Hämmerle et al. 2004). Die Vorteile einer späten Implantation liegen in der ausgeheilten Alveole mit den darüber liegenden Weichgeweben, so dass eine Lappenpräparation vorgenommen werden kann.

Nachteile sind die längere Behandlungszeit sowie der eventuelle Rückgang von Knochenvolumen im Implantationsgebiet (Hämmerle et al. 2004). 
Man unterscheidet außerdem zwischen sofort belasteten und spät belasteten Implantaten. Da die Osseointegration eines Implantates maßgebend von der ungestörten Einheilungsphase abhängt, kann eine frühe Belastung zu einer geringeren Einheilung oder zum Misserfolg der Implantation führen. Bei Planung einer direkten Belastung des Implantates nach Insertion sollte eine besonders hohe Primärstabilität gegeben sein sowie eine regelmäßig Okklusionskontrolle stattfinden, um eine Überlastung zu vermeiden (Parithimarkalaignan und Padmanabhan 2013).

Es gibt Implantate in sehr verschiedenen Längen und Durchmessern, angepasst an die verschiedenen Behandlungsansprüche. Auf dem Markt gibt es heute mehrere tausend Implantatsysteme, die sich in der Form der Implantate, die zumeist zylinderförmig oder konisch geformt sind, unterscheiden (Jokstad et al. 2003). Die Implantatsysteme unterteilen sich in Systeme mit einteiligen oder mehrteiligen Implantaten (Binon 2000). Die einteiligen Implantate ragen mit einem Implantataufbau nach Insertion direkt durch die Schleimhaut in die Mundhöhle. Mehrteilige Systeme haben den Vorteil, dass das Implantat nach Insertion mit einer Abdeckschraube verschlossen wird und unter der Schleimhaut belastungsfrei einheilen kann. Nach der Einheilung erfolgt dann die Versorgung des Implantates mit einem Abutment. Bei gleicher Einheilungsphase konnte kein signifikanter Unterschied in der Überlebensrate der beiden Implantatsysteme festgestellt werden (Jokstad et al. 2003; Buser et al. 1991). Mit den ein- oder mehrteiligen Implantatsystemen unterscheiden sich auch die Einheilungsformen der Implantate. Bei einem einteiligen System erfolgt die Einheilung offen bzw. transgingival und bei einem zweizeitigen Operationsverfahren mit einem mehrteiligen System erfolgt die Einheilung geschlossen.

In Hinblick auf eine bessere Oberflächenbenetzbarkeit und die damit folgende begünstigte Implantateinheilung werden die Implantatoberflächen mit unterschiedlichen Techniken modifiziert. Dabei werden die Maschinierung, Beschichtung und das Aufrauen der Oberfläche differenziert. Das Aufrauen findet durch Säureätzung oder Sandstrahlung bzw. durch eine Kombination dieser Maßnahmen statt. Die Beschichtung der erwerblichen Implantate ist sehr vielseitig. Es handelt sich dabei hauptsächlich um die Beschichtung mit titan-plasmaflamespray (TPS), Hydroxylapatit (HA) und mit strukturierten Oxiden.

Implantate mit sandgestrahlter und säuregeätzter Oberfläche mit zusätzlicher Fluoridbeschichtung zeigen eine geringere Zytotoxizität und somit eine bessere 
Biokompatibilität gegenüber anderen Modifikationen. In vivo wurde eine verbesserte periimplantäre Knochenformation in einer frühen Phase der Osseointegration festgestellt (Schliephake et al. 2012).

\subsection{Implantologische Behandlungsplanung und Diagnostik}

Der Einsatz von Implantaten ist bei Fehlen eines Zahnes oder mehrerer Zähne indiziert. Die Behandlungsplanung setzt fundierte Kenntnisse über das Implantatlager voraus. Die klinische Untersuchung und Aufnahme der Anamnese kann eine dreidimensionale Bildgebung indizieren, wenn eine deutliche anatomische Abweichung von der Norm vorliegt. Andererseits kann sich in Folge einer zweidimensionalen Übersichtsaufnahme die Indikation zur dreidimensionalen Bildgebung stellen, wenn pathologische Veränderungen sichtbar sind, deren Ausdehnung oder Lokalisation in der zweidimensionalen Bildgebung nicht eindeutig erfassbar sind. Des Weiteren ist eine dreidimensionale Aufnahme angezeigt, wenn anatomische Nachbarstrukturen nicht beurteilbar sind oder morphologische Besonderheiten aufweisen (Nitsche et al. 2012).

Die Basisuntersuchung sieht zunächst die palpatorische Untersuchung des Alveolarkammes und eine Übersichtsaufnahme in Form eines Orthopantomogrammes (OPG) vor (Ibrahim et al. 2013). Der Vergrößerungsfaktor der OPG-Aufnahme kann durch eine genormte Metallkugel in der Aufnahme berechnet werden. Die korrekte Positionierung des Patienten im Röntgengerät ergibt reproduzierbar genaue Ergebnisse (Vazquez et al. 2008). In der OPGAufnahme kann die vertikale Knochenhöhe und die Nähe zu angrenzenden Strukturen wie dem Canalis mandibulae im Unterkiefer und dem Sinus maxillaris im Oberkiefer beurteilt werden. Über die Knochendichte und -qualität kann in diesem röntgenologischen Verfahren nur begrenzt eine Aussage getroffen werden.

Anhand der genormten Metallkugel können der maximale Durchmesser und die maximale Länge des geplanten Implantates bestimmt werden (Ibrahim et al. 2013). Der Abstand zu einem Nachbarzahn oder einem benachbarten Implantat sollte 1-1,5 mm betragen. Die Distanz zu angrenzenden Strukturen sollte min. 1-2 mm betragen, um das Risiko einer Verletzung zu minimieren (DelBalso et al. 1994). 
Ist die eindeutige Identifikation des Nervenverlaufs im Unterkiefer und damit die Planung der Implantatlänge nicht möglich, sollte eine weiterführende Bildgebung eingesetzt werden. Hierzu können die dreidimensionalen Verfahren digitale Volumentomographie (DVT) oder die Computertomographie (CT) eingesetzt werden. Beide bieten eine hochauflösende Darstellung von Hartgeweben und können die anatomischen Strukturen überlagerungsfrei in allen drei Raumebenen darstellen (Schulze et al. 2009).

CT und DVT unterscheiden sich hauptsächlich durch unterschiedliche Nutzstrahlenfelder und den Aufnahmemodus. Bei der CT wird der Patient durch ein Strahlenfeld transportiert und in einzelnen Schichten aufgenommen, die später zu einem dreidimensionalen Bild errechnet werden. Die DVT-Technik arbeitet hingegen mit einem kegelförmigen Strahl, der sich mit einem gegenüberliegenden Detektor um den Patienten bewegt und eine vollständige Rotation zur Bildaufnahme benötigt (Howerton und Mora 2008). Für eine DVT-Aufnahme kann zur untersuchten Region ein passendes Aufnahmevolumen ausgewählt werden, so dass nur die diagnostisch relevante Region aufgenommen wird (Harris et al. 2012). Daraus resultieren im Vergleich zur CT-Technik eine kürzere Aufnahmezeit und eine verringerte Strahlenbelastung (Mohan et al. 2011). Die dreidimensionalen Bildgebungsverfahren bieten die Möglichkeit einer computernavigierten Behandlungsplanung und Implantatinsertion. Die Implantatposition kann anhand einer Software geplant und virtuell der Durchmesser und die Länge des Implantates bestimmt werden (Neugebauer et al. 2006). Mit Hilfe der CAD/CAMTechnologie können darüber hinaus Bohrschablonen, Langzeitprovisorien und definitiver Zahnersatz hergestellt werden (Harris et al. 2012). 


\subsection{Zahnärztliche Röntgentechniken}

\subsubsection{Zweidimensionale Röntgenverfahren}

\subsubsection{Konventionelle Zahnfilmaufnahmen}

Die konventionelle intraorale Radiologie basiert auf einen Film-Folien-System. Hierbei können ein bis drei benachbarte Zähne gemeinsam aufgenommen werden. Diese Technik stellt in zwei Dimensionen sowohl die mesio-distale wie auch die okklusal-apikale Distanz eines Zahnes dar (Garg und Vicari 1995). Durch die Röntgenstrahlung werden die unterschiedlich dichten Strukturen der Zähne und des Knochens durchdrungen und die Folie des Röntgenbildes unterschiedlich stark belichtet. In den stärker belichteten Bereichen wird die Silberkörnung aus der Emulsion, die die Folie des Filmes beschichtet, reduziert. Im Prozess der Bildentwicklung wird diese Körnung herausgewaschen und auf der Folie entsteht ein sichtbares Bild der Zähne und der Knochenstruktur (Van der Stelt 2008). Diese Röntgentechnik zeigt eine reproduzierend hochauflösende Bildqualität bei geringer Strahlenbelastung. Die einzelnen Zahnstrukturen und das umliegende Knochengewebe können gut beurteilt werden (Corpas et al. 2011). Pathologische Veränderungen in der periapikalen Region oder Kariesläsionen können zuverlässig detektiert werden (Raghav et al. 2010; Valizadeh et al. 2012). Intraorale Zahnfilme können zur Verlaufskontrolle des marginalen Knochens um ein Implantat genutzt werden. Durch das Verwenden von Filmhaltern kann ein Bild in derselben Position wiederholt werden und eine adäquate Übersicht über den Knochenverlauf über einen langen Zeitraum bieten (Vandenberghe et al. 2008). Nachteilig sind die durch die zweidimensionale Technik bedingten Überlagerungen von anatomischen Strukturen. Durch sie können beispielsweise vertikale Knocheneinbrüche oder interne Resorptionen des Zahnes schwer erkannt werden (Scarfe et al. 2009). Die Form des Kieferknochens im Oberkiefer kann auf Grund der Wölbung zu geometrischen Verzerrungen führen, so dass die abgebildeten Strukturen nicht mehr adäquat proportioniert auf dem Bild wiedergegeben werden (Vandenberghe et al. 2008; Corpas et al. 2011). Anwendung finden die Zahnfilmaufnahmen in der Kariesdiagnostik, der endodontischen Diagnostik und der Verlaufskontrolle nach abgeschlossener Obturation des Zahnes sowie in 
der Parodontologie zur Beurteilung von horizontalen und vertikalen Knochendefekten (Vandenberghe et al. 2008; Haghanifar et al. 2014; Krzyżostaniak et al. 2014).

\subsubsection{Digitale Zahnfilmaufnahmen}

Die digitale Röntgentechnik wurde in den 1980er Jahren in der Zahnmedizin eingeführt (Van der Stelt 2005). Die konventionellen Zahnfilme werden heute noch verwendet und wurden nicht durch die digitalen Systeme gänzlich verbannt.

Zwischen den beiden Systemen unterscheidet sich lediglich die Speicherart des Bildes. Die Röntgenquelle als Tubus bleibt erhalten. Die Dimensionen des Filmes bleiben gleich und ebenso die Vor- und Nachteile dieser zweidimensionalen Aufnahmetechnik gelten in gleicher Weise für die digitale Zahnfilmaufnahme. Weitere Vorteile gegenüber dem konventionellen Zahnfilm liegen in der geringeren Strahlendosis, die zur Aufnahme benötigt wird und in der nachträglichen Bearbeitungsmöglichkeit des digitalen Röntgenbildes durch eine Computersoftware (Van der Stelt 2005). Das Röntgenbild kann bei der Bearbeitung vergrößert werden und der Kontrast sowie die Grautöne können verändert werden. In der Software gibt es verschiedene voreingestellte Bearbeitungsoptionen zur Wahl, die z. B. die Kariesdiagnostik erleichtern sollen (Sitzmann und Benz 2000). Es unterscheiden sich zwei Speichermethoden in der dentalen, digitalen Radiographie: Die direkte und die indirekte Radiographie. Bei der direkten digitalen Zahnfilmaufnahme wird anhand eines Halbleitersensors (CCD-Sensor, charged-coupled device) das Bild aufgenommen und direkt über eine Kabelverbindung auf einen Computer übertragen (Sitzmann und Benz 2000).

Die Röntgenstrahlung trifft auf den Sensor und durch einen Szintillator wird die Energie der Röntgenstrahlung in sichtbares Licht umgewandelt. Im Anschluss können die unterschiedlichen Lichtintensitäten als elektrische Signale auf den Computer übertragen und in Form eines Röntgenbildes dargestellt werden. Der Sensor kann auch mit der CMOSTechnologie (complementary metal-oxide-semiconductor) versehen sein. Diese Technologie unterscheidet sich darin, dass der Sensor selbst die Transformation der Strahlenenergie in sichtbares Licht übernimmt. Damit ist er in der Herstellung kostengünstiger. 
Die indirekten digitalen Zahnfilmaufnahmen werden mit Hilfe einer Speicherfolie aufgenommen. Diese sogenannten PSP-Folien (photo-stimulable phosphor) sind mit Phosphorkristallen beschichtet. Sie können die eintreffende Energie der Röntgenstrahlen für eine gewisse Zeitspanne speichern (Van der Stelt 2005). In einen speziellen Scanner wird die Speicherfolie nach der Aufnahme durch einen Laserstrahl mit einer bestimmten Wellenlänge abgetastet. Die gespeicherte Energie der Röntgenstrahlung wird in Form von Licht einer anderen Wellenlänge freigesetzt. Ein Fotodetektor transformiert dieses Licht in elektrische Signale und leitet sie an den Computer, auf dem mit Hilfe einer Software das Röntgenbild dargestellt wird (Barbat und Messer 1998). Im Anschluss ist das vorherige Bild gelöscht und der Film kann wieder verwendet werden. Die direkte Aufnahmetechnik ist mit einem Kabel an das Computersystem angeschlossen. Hierdurch wird das System unflexibel und weniger tolerabel für den Patienten, da auch der Sensor starr ist und eine größere Schichtstärke hat als ein konventioneller Zahnfilm. Vorteilig ist dem gegenüber das Speicherfoliensystem. Die Maße des Filmes ähneln denen eines konventionellen Films (Van der Stelt 2005). 


\subsubsection{Dreidimensionale Röntgenverfahren}

\subsubsection{Digitale Volumentomographie (DVT)}

Die digitale Volumentomographie (DVT) ist eine moderne, röntgenologische Aufnahmetechnik zur Herstellung von dreidimensionalen Bildern des Kopf- und Halsbereiches. Sie wurde Ende der neunziger Jahre entwickelt (Patel 2009) und 2001 mit dem ersten DVT-Gerät New Tom QR DVT 9000 der Firma Quantitative Radiology S.r.l. (Verona, Italien) eingeführt. Seither folgten weitere Geräte verschiedener Hersteller (Scarfe 2006).

Dieses Röntgenverfahren zeichnet sich durch eine hochauflösende Darstellung von Hartgeweben, die Verwendung eines dreidimensionalen Nutzstrahlenbündels und eine isometrische Ortsauflösung aus (Schulze et al. 2009). Während der Aufnahme sitzt, steht oder liegt der Patient und die Strahlenquelle rotiert mit dem gegenüberliegenden Detektor um den Patienten (Mohan et al. 2011). Die Aufnahme wird durch eine vollständige Rotation des Gerätes um den Patienten generiert. Die Strahlenquelle in Form eines kegelförmigen Röntgenstrahles liegt gegenüber einem Bilddetektor. Während der Rotation um den Patienten entsteht eine große Anzahl von einzelnen Projektionsaufnahmen. Die Summe der erfassten Projektionsaufnahmen sowie deren Rückprojektionen werden durch einen spezifischen Algorithmus vom Computer zu einen dreidimensionalen Rohdatensatz errechnet (Scarfe 2006; Harris et al. 2012). Im Anschluss an die Rekonstruktion der Projektionsaufnahmen wird das dreidimensionale Bild in einem Software-Programm in der sagittalen, koronalen und transversalen Schicht dargestellt (Scarfe 2006).

Im Vergleich mit der konventionellen Computertomographie (CT) wird bei der DVT durch eine vollständige Rotation des kegelförmigen Strahles direkt ein dreidimensionales Volumen der aufgenommenen Region auf dem Detektor erzeugt. Bei der CT-Aufnahme wird der Patient schichtweise durch ein fächerförmiges Strahlenfeld transportiert und die entstandenen zweidimensionalen Schichtaufnahmen werden zu einem dreidimensionalen Datensatz errechnet (Howerton und Mora 2008). Es resultiert eine geringere Strahlenexpositionszeit für den Patienten in der DVT. Die Bestrahlungszeit beträgt durchschnittlich ca. 18 Sekunden, ein Siebtel der Zeit eines konventionellen CT-Gerätes (Mohan et al. 2011).

Die aktuell erhältlichen DVT-Geräte unterscheiden sich in den technischen Aufnahmeparametern, wie dem Aufnahmevolumen (field of view, FOV), der Software und der 
Detektortechnologie (Bildverstärker, flatpanel detector) (Howerton und Mora 2008). Weiterhin kann die Patientenposition (stehend, liegend, sitzend) und die Ortsauflösung bei den unterschiedlichen Geräten variieren (Nitsche et al. 2012). Das Aufnahmevolumen kann dem Untersuchungsfeld angepasst werden. Hauptsächlich wird zwischen kleinem, mittleren und großem Aufnahmevolumen unterschieden. Die Größe des maximalen und minimalen FOV ist bei den erhältlichen DVT-Geräten nicht einheitlich. Teils kann die Größe des FOV variabel an das Untersuchungsfeld angeglichen werden. Bei einem Fokus auf die Kiefer und die dentalen Strukturen wird ein kleineres oder mittleres Aufnahmevolumen gewählt. Bei einer diagnostischen Fragestellung, die den Mund-, Kiefer- und Gesichtsbereich betrifft ist ein großes FOV vorzuziehen (Harris et al. 2012). Die Größenauswahl des FOV wirkt sich auf die Bildgenauigkeit der DVT-Aufnahme aus. Kleinere und mittlere FOV liefern eine bessere Detaildarstellung unterschiedlicher Strukturen als ein großes Aufnahmevolumen. Der relative Qualitätsverlust bei einem großen Aufnahmevolumen steht einer höheren Neigung zu Bewegungsartefakten bei kleineren Aufnahmevolumina gegenüber (Hassan et al. 2010). Die Qualität einer DVT-Aufnahme hängt zu einem großen Teil von der Bildauflösung ab (Hassan et al. 2010). Charakteristisch für DVT-Aufnahmen ist eine isometrische Ortauflösung in allen drei Raumebenen (Schulze et al. 2009). Die kleinste Bildeinheit, ein Voxel, ist dabei ein in allen drei Raumebenen gleich großes kubisches Element (Schulze et al. 2010). Die Voxelgröße variiert abhängig vom Gerät zwischen $0,4 \mathrm{~mm}$ und 0,125 mm (Patel et al. 2009a).

In der Detektortechnologie unterscheidet man zwischen Systemen mit Bildverstärkern (image intensifier detector, IID) oder Flächendetektoren (flatpanel detector, FPD) (Hassan et al. 2010). Die beiden Detektorarten sind der Röntgenstrahlung gegenüber kaum sensitiv. Daher brauchen beide Detektorsysteme einen vorgeschalteten Transformator, der die Röntgenstrahlung in sichtbares Licht umwandelt. Das sichtbare Licht kann folgend die Sensoren auf dem Detektorfeld aktivieren. In den erhältlichen Geräten finden sich die Kombination von einem Bildverstärker und einer CCD-Kamera (charged-couple-device) zur Aufnahme der Bilder oder ein flatpanel detector und ein Szintillator zur Transformation der Röntgenstrahlung in sichtbares Licht. Das erste System zeichnet sich durch eine einfache Bauweise und Steuerung aus. Das pixelweise Auslesen des Sensors, eine geringe maximale Bildrate und eine schlechte Ortsauflösung durch die Bildverstärkung sind die Nachteile des IID-Systems (Böhm 2007). Das System des flatpanel detector erzeugt durch einen Szintillator 
sichtbares Licht. Dabei bleibt eine Lichtstreuung aus. Es wird eine sehr gute Ortsauflösung erzielt. Während des Auslesevorganges können weitere Daten aufgenommen und verarbeitet werden, ohne dass es zu einer Artefaktbildung durch Verzögerungen bei der Aufnahme kommt (Krause 2013). Zudem wird eine größere Fläche des Detektors von bis zu 43 x $43 \mathrm{~cm}$ möglich (Lindner 2001; Böhm 2007). Nachteile dieses Systems sind die im Vergleich zum Bildverstärkersystem nötige höhere Strahlendosis und die kostenintensivere Herstellung (Krause 2013). Generell sollte die dreidimensionale Aufnahmetechnik der Digitalen Volumentomographie als eine ergänzende Röntgentechnik in der Zahnmedizin angesehen werden. Aus Gründen des Strahlenschutzes, des ALARA-Prinzips (as low as reasonably achievable) sollte ein Patient der höheren Röntgenstrahlung nur mit rechtfertigender Indikation ausgesetzt werden (Harris et al. 2012).

Die Indikation zur DVT-Aufnahme stellt sich, wenn die Fragestellung, die eine Bildgebung erfordert, nicht adäquat durch eine weniger strahlenbelastende, konventionelle Röntgenmethode beantwortet werden kann (European Commission of Energy 2012). In den einzelnen Fachgebieten der Zahnmedizin ergeben sich unterschiedliche Indikationen, bei denen eine DVT-Untersuchung erfolgen kann:

In der Zahnerhaltung kann die zusätzliche Untersuchung in gewissen Behandlungssituationen durch eine dreidimensionale Bildgebung in den Gebieten der Endodontie und Parodontologie sinnvoll sein. Für die Kariesdiagnostik, besonders in Nachbarschaft zu metallbasiertem Zahnersatz, ist die DVT-Technik nicht geeignet (Zhang et al. 2007). In der Endodontie kann die dreidimensionale Wurzel- und Kanalanatomie in den zweidimensionalen Aufnahmetechniken nur begrenzt dargestellt werden (Matherne et al. 2008; Stavropoulos und Wenzel 2007; Patel 2009). Anatomische Anomalien, periapikale Pathologien sowie Wurzelfrakturen und -resorptionen infolge eines dento-alveolären Traumas können nicht überlagerungsfrei dargestellt werden. Die Detektion von periapikalen Läsionen kann in der DVT-Aufnahme früher und mit einer sichereren Lokalisation durchgeführt werden als in einer konventionellen intraoralen Aufnahme (Estrela et al. 2008). Die Beurteilung von apikalen Läsionen in enger Nähe zur Kieferhöhle oder zum Canalis mandibulae können in zweidimensionalen Verfahren meist nicht exakt abgebildet werden. Bei einem chirurgischen, periapikalen Eingriff stellt die dreidimensionale DVT-Aufnahme die Beziehung der benachbarten anatomischen Strukturen und der pathologischen Läsion mit einer höheren 
Sensitivität dar als konventionelle Röntgenverfahren (Patel et al. 2009b; Low et al. 2008). Durch Traumata entstandene Wurzelfrakturen sind in den konventionellen Bildgebungsverfahren in ihrer Ausdehnung, eventueller Luxation, Dislokation oder Alveolarfortsatzfrakturen häufig schwer zu beurteilen (Cohenca et al. 2007). Bei Wurzelresorptionen kann durch den asymptomatischen und langsamen Verlauf in zweidimensionalen Röntgenverfahren erst spät eine eindeutige Diagnose getroffen werden. Die DVT-Aufnahme kann in diesen Fällen ein hilfreiches diagnostisches Verfahren sein (Scarfe et al. 2009) und die Entscheidung des operativen Zuganges und dessen Ausdehnung beeinflussen. In der Parodontologie ist die Beurteilung von Knochenabbau durch die zweidimensionalen Röntgenverfahren nur im interdentalen Bereich mesial und distal möglich.

Die Beurteilung des lingualen/palatinalen und bukkalen Knochens ist durch Überlagerungen nicht eindeutig möglich. Allgemein stellt die intraorale Zahnfilmaufnahme den diagnostischen Goldstandard zur Bestimmung von Knochendefekten dar. Die Erfassung und Messung von singulären, vertikalen Knocheneinbrüchen und Furkationsbefunden kann jedoch durch eine dreidimensionale DVT-Aufnahme exakter erfolgen als durch zweidimensionale Bildgebungsverfahren (Vandenberghe et al. 2007). In der zahnärztlichen Prothetik ist eine Beurteilung von prothetischen Pfeilern in einer DVT-Aufnahme denkbar, wenn die klinische Untersuchung und die zweidimensionale Bildgebung keine eindeutige Therapieplanung zulassen. Fachübergreifend zur zahnärztlichen Chirurgie kann anhand einer DVT-Aufnahme vorhandenes Knochenangebot beurteilt werden. Die Beurteilung der Knochenqualität in hounsfield units (HU) ist in DVT-Aufnahmen nicht möglich (Corpas et al. 2011). In den Aufnahmen ist die Knochendichte nicht zuverlässig bestimmbar, da die Aufnahmeprotokolle nicht einheitlich sind. Somit sind die Aufnahmeparameter, Patientenpositionierung und Gerätewerte unter den Herstellern starken Schwankungen unterworfen. Die Knochendichte kann daher nur im CT bestimmt werden (Hiasa et al. 2011; Nackaerts et al. 2011). Die dreidimensionale Planung von Implantaten, Zahnersatz oder eine computernavigierte Versorgung mit Implantaten ist mit einer DVT-Aufnahme möglich (Neugebauer et al. 2006; Braut et al. 2011; Inokoshi et al. 2012). Die dreidimensionalen Daten erlauben des Weiteren eine Verknüpfung zu CAD/CAM-Systemen zur Herstellung von Bohrschablonen zur Implantatinsertion, Langzeitprovisorien oder definitivem Zahnersatz (Schulze et al. 2009; Harris et al. 2012). In der Kiefergelenks- und Funktionsdiagnostik besteht eine Indikation zur 
DVT-Aufnahme bei primär knöchern bedingten Pathologien des Kiefergelenkes. Veränderungen des Weichgewebeanteils des Kiefergelenkes, einschließlich des Diskus articularis, können in der DVT-Aufnahme nicht eindeutig beurteilt werden (Schulze et al. 2013). In der Implantologie ist vor jeder Implantation eine ausreichende Diagnostik vorauszusetzen. Bietet eine zweidimensionale Röntgenaufnahme hierbei keine suffiziente Beurteilung des vorhandenen Knochenangebotes, der Knochenstruktur und der angrenzenden anatomischen Strukturen, sollte eine zusätzliche dreidimensionale Aufnahmemethode in Betracht gezogen werden (van Assche et al. 2007; Nitsche et al. 2012). Die Bildqualität verschlechtert sich in der DVT-Technologie, wie auch in der CT-Technologie, wenn metallische Körper in der aufzunehmenden Region vorhanden sind. Im rekonstruierten Datensatz treten Bildartefakte um die metallischen Körper auf. Es wird zwischen vielen Arten und Ursachen von Artefakten in DVT-Aufnahmen unterschieden. Besonderen Einfluss auf die Bildqualität haben die von metallischen Körpern hervorgerufenen Aufhärtungsartefakte. Durch die Aufhärtungen und Projektionslinien um ein Implantat, metallischen Zahnersatz oder einen Wurzelstift verschlechtert sich die Bildqualität der Aufnahme in diesem Bereich (Nabha et al. 2014; Schulze et al. 2011). Im rekonstruierten Bild zeigt sich das Metall stark belichtet, das angrenzende Gewebe ist zum Teil vom Metall überstrahlt und schlecht beurteilbar. Im Aufnahmeprozess der DVT-Aufnahme durchdringen nur Röntgenstrahlen mit besonders hoher Intensität, d.h. mit besonders hoher Energie, die dichten Metallkörper. Der Sensor speichert dabei für den Bereich des Metallkörpers eine sehr hohe Energiedichte. Im Rekonstruktionsprozess wird die Bilderzeugung verändert und es resultiert ein starker Kontrast zwischen dem sehr hohen Dichte des Metalls und dem gemäßigt dichten Gewebe um den Metallkörper. Durch den Rekonstruktionsalgorithmus und damit einhergehender fehlerhafter Rückprojektionen entsteht in der späteren Aufnahme eine Verschlechterung der Bildqualität im Bereich des Metallkörpers. In direkter Nachbarschaft zum Implantat entsteht ein Effekt, der fälschlicherweise als fehlender Knochen interpretiert werden kann. Hierbei spricht man auch von einem missing data problem, ausgelöst durch den fehlerhaften Rekonstruktionsprozess (Schulze et al. 2010; Schulze et al. 2011). Somit muss im Bereich von metallischen Körpern in einer DVT-Aufnahme mit einer verschlechterten Bildqualität gerechnet werden. Eine genaue Beurteilbarkeit des benachbarten Gewebes kann eingeschränkt oder nicht möglich sein (Schulze et al. 2011). Allgemein stellt die DVT-Aufnahme eine sehr detaillierte Bildgebung zur Implantatplanung dar. Verlaufskontrollen nach Implantation 
sollten aus bildgebenden und strahlenschutzbedingten Gründen weiterhin mit konventionellen Röntgenverfahren durchgeführt werden (Corpas et al. 2011; Schulze et al. 2013; Scarfe 2006).

\subsection{Strahlenbelastung}

Im letzten Jahrzehnt hat die Anwendung von dreidimensionalen Bildgebungsverfahren im Bereich der Mund-, Kiefer- und Gesichtschirurgie deutlich zugenommen. Sowohl CTAufnahmen als auch DVT-Aufnahmen gehören häufig zur präoperativen Planung. Die digitale Volumentomographie wird oft als strahlenärmere Röntgenmethode im Vergleich zur Computertomographie dargestellt. Bisher gibt es jedoch keine einheitlichen Aufnahmeprotokolle für DVT-Aufnahmen sowie Dosisprotokolle zur Minimierung der Strahlenbelastung bei gleichbleibender Bildqualität, wie es sie bereits für multislice-CT (MSCT) gibt (Loubele et al. 2008; Hiasa et al. 2011; Nackaerts et al. 2011). Der CTDI (computed tomography dose index) zeigt die Strahlenbelastung eines CT-Gerätes an. Durch ein genormtes CTDI-Phantom kann die Strahlung gemessen werden, die über eine Länge von $10 \mathrm{~cm}$ im Zentrum des Phantoms auftrifft. Dieser Index kann für die DVT-Aufnahmen nicht verwendet werden, da die Länge von 10cm die Streustrahlung des DVT-Strahles nicht erfassen würde und falsche Ergebnisse aus der Dosismessung resultieren würden (Loubele et al. 2008). In der Literatur finden sich unterschiedliche Angaben zur effektiven Dosis bei der DVTAufnahme. In der Stellungnahme der Deutschen Gesellschaft für Implantologie variiert die effektive Strahlendosis zwischen 11-674 $\mu$ Sv für eine DVT-Aufnahme (Nitsche et al. 2012). Ludlow et al. differenzieren folgend zwischen den einzelnen field of view: 46-1073 $\mu \mathrm{Sv}$ (großes FOV), 9-560 $\mu \mathrm{Sv}$ (mittleres FOV) und 5-562 $\mu$ Sv (kleines FOV) (Ludlow et al. 2015). Im Vergleich beträgt die effektive Strahlendosis einer CT-Aufnahme etwa 180-2100 $\mu$ Sv. In Vergleichsstudien zur Strahlendosis von CT- und DVT-Geräten waren die effektiven Dosiswerte für die DVT-Geräte trotz großer Geräteunterschiede geringer als auch bei low-multislice-CT-Geräten und zeigten eine bessere Bildqualität (Loubele et al. 2009; Hofmann et al. 2013). Eine Zahnfilmaufnahme gilt als gering strahlenbelastend mit einer effektiven Dosis von 1-8,3 $\mu$ Sv (European Commission of Energy 2003, 2012; Nitsche et al. 2012). 


\subsection{Fragestellung}

Die dreidimensionale Röntgentechnik der digitalen Volumentomographie (DVT) ist in den letzten Jahrzehnten in der Zahnmedizin zu einem immer wichtigeren diagnostischen Verfahren herangewachsen. Durch eine gute Ortsauflösung und eine überlagerungsfreie Darstellung von Hartgeweben sowie ein wählbares Aufnahmevolumen ist eine Aufnahme ähnlich der Qualität einer CT-Aufnahme bei geringerer Strahlenbelastung möglich.

In dieser Untersuchung sollte anhand von digitalen und konventionellen Zahnfilmaufnahmen sowie von dreidimensionalen DVT-Aufnahmen die Darstellbarkeit von Implantaten mit unterschiedlichen Defekten, iatrogen gesetzten Knochendefekten und verschiedenen Fremdmaterialen evaluiert werden. Nachfolgend an eine vorrangegangene Studie (Jarnot 2011) evaluierten in dieser Studie zehn Probanden mit gleichem Wissens- und Ausbildungstand zahnärztliche Röntgenaufnahmen unter dem Aspekt der Vergleichbarkeit von zwei- und dreidimensionalen Verfahren. Die Studie bearbeitet die Hypothese, dass die dreidimensionale digitale Volumentomographie exaktere und bessere Informationen über eine Implantat-Knochensituation liefert als konventionelle und digitale zweidimensionale Zahnfilmaufnahmen. Es wurde verglichen, ob Implantate und ihr umliegendes Gewebe sowie Fremdmaterialien und Defekte im Knochengewebe und an den Implantatkörpern sowie deren räumliche Zuordnung in den drei radiologischen Verfahren exakt beurteilt werden können. 


\section{Material und Methoden}

\subsection{Versuchsdurchführung}

\subsection{1 Überblick über die Versuchsdurchführung}

In der vorliegenden Untersuchung wurden drei zahnärztliche radiologische Verfahren auf ihre Darstellbarkeit von Knochenstrukturen und enossalen Implantaten evaluiert. Die verwendeten Präparate von Schweinekiefern sind mit zahnärztlichen Implantaten versehen. In den Präparaten finden sich unterschiedliche Defekte an Knochen und Implantaten sowie Fremdkörper unterschiedlicher Art und Dichte. Jedes Präparat wurde in den drei radiologischen Verfahren aufgenommen und folgend durch zehn Untersucher beurteilt. Die verwendeten Schweinekieferpräparate stammen aus einer früheren Untersuchung durch Dr. Lena Jarnot (Jarnot 2011). In der vorrangegangenen Untersuchung inserierte Frau Dr. Jarnot in die ursprünglich vierzehn Schweinekieferpräparate jeweils ein enossales Implantat. Die Präparate wurden mit unterschiedlichen Defekten versehen. Hierzu gehören Knochendefekte in Form von iatrogenen Alveolardefekten, Defekte an den Implantatkörpern sowie die Einlagerung von Fremdmaterialien in die Implantatbetten und in das umliegende Knochengewebe. In der vorliegenden Studie wurden zunächst von den allen Schweinekiefermodellen konventionelle Zahnfilme hergestellt und aus den vorhandenen vierzehn Schweinekieferpräparaten zehn Präparate ausgewählt. Bei der Auswahl war das Gleichgewicht zwischen offensichtlich erkennbaren und durchaus schwierig erkennbaren Defekten und Fremdmaterialien entscheidend. In Tabelle 1, Seite 28 finden sich die Zuordnung der einzelnen Präparate und die vorliegenden Defekte und Fremdmaterialen.

Nach Auswahl der zehn Präparate erfolgten von diesen ebenfalls digitale Zahnfilmaufnahmen und Aufnahmen mit der digitalen Volumentomographie. Im Anschluss wurden die Aufnahmen in randomisierter Reihenfolge durch 10 Untersucher anhand von Prüfbögen befundet und danach statistisch ausgewertet. 


\subsubsection{Flussdiagramm der Versuchsdurchführung}

14 Schweinekieferpräparate mit enossalen Implanaten und unterschiedlichen Arten von Defekten und Fremdkörpern
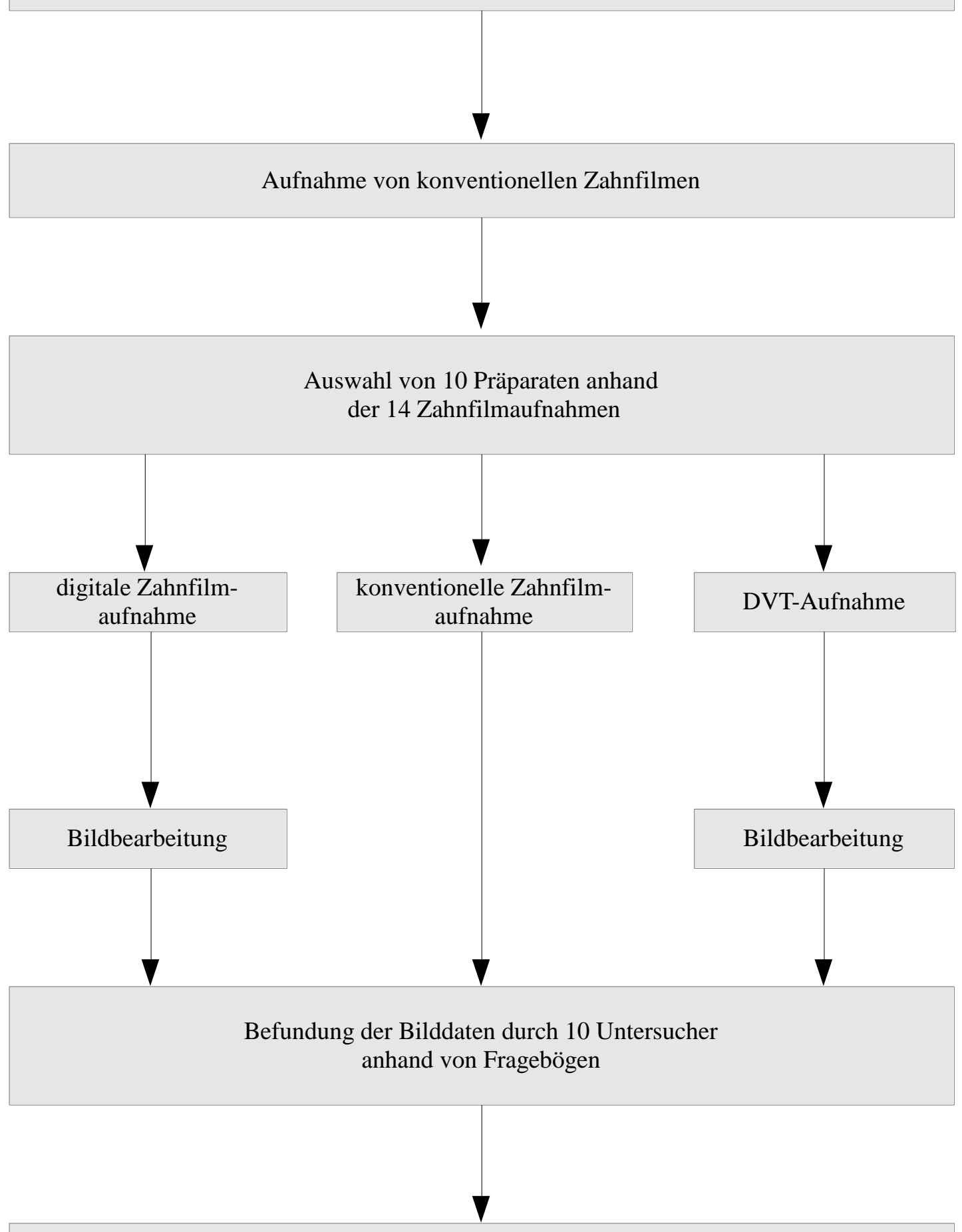

Evaluation der Daten und statistische Auswertung 


\subsection{Materialliste}

Im Rahmen der Versuchsdurchführung wurden folgende Materialien verwendet:

- Schweinekiefermodelle mit inserierten Implantaten (Jarnot 2011, Georg-AugustUniversität Göttingen, Deutschland)

- konventionelles Zahnfilmgerät Heliodent MD (Sirona Dental Systems, Bensheim, Deutschland)

- digitales Zahnfilmgerät Miniray ( SOREDEX, Helsinki, Finnland)

- Vista scan mini plus, Scan-Gerät für die Speicherfolien des digitalen Zahnfilmgerätes (Dürr Dental, Bietigheim-Bissingen, Deutschland)

- Speicherfolie 2+ black, Größe 2; 3 x 4 cm (Dürr Dental, Bietigheim-Bissingen, Deutschland)

- Digitale Volumentomographie PaX-Zenith 3D (Orangedental- VATECH Co, Ltd \& E-Woo, Hwaseong, Südkorea)

- Röntgenfilm Kodak Insight IP 21; 30,5 x 40,5 mm (Carestream Health Inc, Rochester, NY, USA)

- Periphery-Wax, Enta (Bergen op Zoom, Niederlande)

- Messlehre AA804R, 150 mm, 6“ Stainless (AESCULAP Dental, Tuttlingen, Deutschland)

- Computersoftware für den digitalen Zahnfilm: Byzz (Orangedental, Biberach, Deutschland)

- Computersoftware für digitale Volumentomographie: Ez3D 2009 (Orangedental, Biberach, Deutschland)

- Befundungsbildschirm Dell P1913s, 19 Zoll (Dell GmbH, Frankfurt am Main, Deutschland) 


\subsection{Schweinekiefermodelle mit enossalen Implantaten}

Die Schweinekiefermodelle liegen bereits aus einer früheren Dissertationsarbeit von Frau Dr. med. Lena Jarnot in der Abteilung Mund-Kiefer-Gesichtschirurgie vor (Jarnot 2011). Als Schweinekiefermodelle dienten halbe Unterkiefer von Schlachttieren (Harzer Fleisch Vermarktung, Schlachthof Göttingen, $\mathrm{GmbH}$ ). In diese wurden 14 Implantate des Systems ITI-Bonefit der Firma Straumann AG (Basel, Schweiz) inseriert. Die Implantate gehören zur Produktreihe Standard Implantat, regular neck, SLA, Material Titan. Es wurden Implantate der Längen $8 \mathrm{~mm}, 10 \mathrm{~mm}, 12 \mathrm{~mm}, 14 \mathrm{~mm}$ und $16 \mathrm{~mm}$ verwendet. Zudem hatten die Implantate verschiedene Durchmesser (3,3 mm, 4,0 mm, 4,1 mm). Die Implantate der Durchmesser 4,0 $\mathrm{mm}$ stammen aus einer älteren Implantatgeneration der Firma Straumann, die sich von den Implantaten der neuen Generation ( auf den Durchmesser unterscheiden. In Bezug auf das Material unterscheiden sie sich nicht voneinander. Die Implantate wurden in der unbezahnten Eckzahnregion der Schweinekiefer inseriert. Dazu fanden zunächst eine Pilotbohrung und eine Erweiterungsbohrung statt, wie sie vom System des ITI-Bonefit vorgegeben sind. Im Folgenden wurde das Gewinde im Knochen manuell mit einem Gewindeschneider präpariert und anschließend das vorgesehene Implantat inseriert. 


\subsection{Defekte an Implantaten, Alveolarknochen und Fremdkörper}

Sowohl die Implantate als auch der Alveolarknochen erhielten vor der Implantation iatrogene

Defekte und zusätzlich wurden Fremdmaterialen in die Implantatbetten eingefügt.

Knochendefekte (8 Präparate):

Das Knochengewebe erhielt Manipulationen mit Hilfe eines konischen Präparationsinstrumentes in $4 \mathrm{~mm}$ Höhe und von $1 \mathrm{~mm}$ Tiefe. Es entstand ein keilförmiger Defekt. Des Weiteren wurde ein walzenförmiges Instrument eingesetzt, um eine Längsrille von $1 \mathrm{~mm}$ Tiefe und einer Länge von $\leq 10 \mathrm{~mm}$ herzustellen. Als weitere Defektart fand eine künstliche Spaltung des koronalen Alveolarknochens statt (Bonesplitting). Der Defekt „Überdimension“ war die vorgebohrte Kavität für ein Implantat der Länge $12 \mathrm{~mm}$. Anschließend wurde jedoch ein Implantat der Länge $8 \mathrm{~mm}$ inseriert.

In einem weiteren Präparat (Defekt: Ersatz, lingual perforiert) wurde in die vorgebohrte Kavität ein überdimensioniertes Implantat inseriert, das durch seine zu großen Ausmaße die linguale knöcherne Begrenzung perforierte. Im Anschluss entfernte man dieses Implantat und ersetzte es durch ein kleineres Implantat (Länge $8 \mathrm{~mm}$, Durchmesser 3,3 $\mathrm{mm}$ ) bei verbleibender Perforation.

Implantatdefekte (6 Präparate):

Vor Insertion der Implantate in die Schweinekieferpräparate fand eine Präparation unterschiedlicher Längs- und Querrillen statt. Diese Defekte wurden mit einer diamantierten Walze produziert. Der Durchmesser betrug dabei $1 \mathrm{~mm}$ und eine Tiefe von 0,5 mm (Die Walze wurde bis zu ihrem größten Umfang versenkt, danach erfolgte eine Nachmessung anhand einer Messlehre). Jeder Defekt hatte eine Länge von $8 \mathrm{~mm}$.

Die Längsrillendefekte dehnen sich über die gesamte Länge des Implantatgewindes aus und enden im Bereich des Implantathalses. Die Querrillendefekte wurden an zwei verschiedenen Positionen an den Implantaten platziert: zwischen den oberen beiden Windungen und zwischen den unteren beiden Windungen. Außerdem entfernte man entweder die oberen beiden oder die unteren beiden Windungen am Implantatgewinde mit einer Lindemannfräse. 
Fremdmaterialien (11 Präparate):

Zusätzlich zu den bereits beschriebenen Defekten wurden einigen Präparaten Fremdkörper zugefügt. Dabei handelte es sich um Edelstahlkugeln mit zwei verschiedenen Durchmessern: $1 \mathrm{~mm}$ und 1,6 mm (Medizinische Werkstätten der Universitätsklinik, Göttingen, rostfreier Stahl). Weiterhin wurde das Knochenersatzmaterial ß-TCP-Keramikpartikel der Körnung 500-1000 $\mu \mathrm{m}$ mit einem Löffel nach Hemmingway nach der Vorbohrung in den Knochen eingebracht und danach das Implantat inseriert. Weitere Fremdkörper sind Guttaperchastifte, die als $1 \mathrm{~mm}$ lange und mit einem Durchmesser von $0,3 \mathrm{~mm}$ in einigen Präparaten vorliegen. In Tabelle 1, Seite 28 finden sich die Präparate mit den zugehörigen Defekten und Fremdmaterialien. 


\begin{tabular}{|c|c|c|c|c|}
\hline $\begin{array}{c}\text { Präparat } \\
\text { Nr. }\end{array}$ & $\begin{array}{l}\text { Durchmesser (D) / } \\
\text { Länge (L) der } \\
\text { Implantate (mm) }\end{array}$ & $\begin{array}{l}\text { Implantatdefekt } \\
\text { (Art, Lokalisation) }\end{array}$ & $\begin{array}{l}\text { Fremdkörper } \\
\text { (Anzahl, Art) }\end{array}$ & $\begin{array}{c}\text { Alveolardefekt } \\
\text { (Art, } \\
\text { Lokalisation) }\end{array}$ \\
\hline 2 & D: 3,3 L: 16 & --- & $\begin{array}{l}\text { 1x Stahlkugel } \\
\varnothing 1 \mathrm{~mm}\end{array}$ & A, mesial \\
\hline 3 & D: 4,1 L: 10 & $\begin{array}{l}\text { B, obere Windung } \\
\text { planiert }\end{array}$ & $\begin{array}{l}\text { ß-TCP, 1x Guttapercha } \\
\text { 30er Point, Länge } 1 \mathrm{~mm} \\
\text { 1x Stahlkugel } \\
\varnothing 1 \mathrm{~mm}\end{array}$ & --- \\
\hline 4 & D: 4,0 L: 16 & ---- & $\begin{array}{l}\text { 1x Stahlkugel } \\
\quad \varnothing 1 \mathrm{~mm}\end{array}$ & --- \\
\hline 5 & D: 4,1 L: 14 & $\begin{array}{l}\text { A, distal des Alveolar- } \\
\text { kammes }\end{array}$ & --- & --- \\
\hline 8 & D: 4,0 L: 14 & $\begin{array}{l}\text { C, zwischen den unteren } \\
\text { zwei Windungen }\end{array}$ & --- & --- \\
\hline 10 & D: 4,1 L: 10 & --- & $\begin{array}{c}\text { 1x Stahlkugel } \\
\varnothing 1 \mathrm{~mm}, 1 \mathrm{x} \text { Stahlkugel } \\
\varnothing 1,6 \mathrm{~mm}\end{array}$ & Bonesplitting \\
\hline 11 & D: 4,1 L: 10 & --- & $\begin{array}{c}\text { 1x Stahlkugel } \\
\varnothing 1,6 \mathrm{~mm}\end{array}$ & --- \\
\hline 12 & D: 4,1 L: 10 & $\begin{array}{l}\mathrm{C} \text {, zwischen den oberen } \\
\text { zwei Windungen }\end{array}$ & $\begin{array}{l}\text { B-TCP, 1x Guttapercha } \\
\text { 30er Point, Länge } 1 \mathrm{~mm}\end{array}$ & $\begin{array}{c}\mathrm{B}, \text { distal } \\
0,5 \mathrm{~mm} \text { tief }\end{array}$ \\
\hline 13 & D: 4,1 L: 8 & --- & $\begin{array}{l}\text { 2x Guttapercha 30er } \\
\text { Point, Länge } 1 \mathrm{~mm}\end{array}$ & Überdimension \\
\hline 14 & D: $3,3 \mathrm{~L}: 8$ & $\begin{array}{l}\text { A, vestibulär des } \\
\text { Alveolarkammes }\end{array}$ & $\begin{array}{c}\text { 1x Stahlkugel } \\
\varnothing 1 \mathrm{~mm} \\
\text { 1x Stahlkugel } \\
\varnothing 1,6 \mathrm{~mm}\end{array}$ & $\begin{array}{l}\text { Ersatz, lingual } \\
\text { perforiert }\end{array}$ \\
\hline
\end{tabular}

Tabelle 1: Zuordnung der Implantatdefekte, Alveolardefekte und Fremdkörper zu den Präparaten

Legende:

Implantatdefekte:

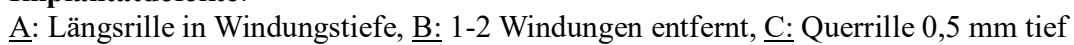

Fremdkörper:

ß-TCP-Keramik: Körnung 500-1000 $\mu$ m, portioniert mit 2 kleinen Löffeln nach Hemmingway

Stahlkugeln: Edelstahlkugeln mit den Durchmessern 1,0 mm und 1,6 mm

Guttapercha: 30er Points mit einem Durchmesser von 0,3 mm, Länge $1 \mathrm{~mm}$

Alvoalardefekte:

A: $1 \mathrm{~mm}$ tiefe, keilförmige Bohrung in $4 \mathrm{~mm}$ Höhe

B: Längsrille von $1 \mathrm{~mm}$ Tiefe und $\leq 10 \mathrm{~mm}$ Länge (Diamantwalze)

zusätzliche Alveolardefekte:

Bonesplitting: künstliche Spaltung des Alveolarkammes bei Implantation

Überdimension, L:12: vorgebohrte Kavität im Verhältnis zum Implantat zu groß, Kavitätenlänge entspricht einer Implantatlänge von $12 \mathrm{~mm}$

Ersatz, lingual perforiert: Perforation bei Implantatinsertion eines im Verhältnis zur Kavität zu großen Implantats; anschließend Insertion eines kleineren Implantats bei bleibender Perforation lingual. 


\subsection{Radiologische Verfahren}

\subsubsection{Zahnfilmaufnahmen}

\subsubsection{Konventionelle Zahnfilmaufnahmen}

Bei der intraoralen Zahnfilmaufnahme wird eine präzise und maßstabsgetreue Röntgenaufnahme eines einzelnen Zahnes und seiner benachbarten Zähne hergestellt.

Der Röntgenfilm liegt oral des abzubildenden Zahnes, möglichst parallel zur Zahnachse. Die Röntgenquelle befindet sich extraoral. Der Röntgentubus muss möglichst nah und senkrecht zur Achse des abzubildenden Zahnes positioniert werden. Idealerweise liegen Zahn und Röntgenfilm parallel zueinander und der Zentralstrahl der Röntgenstrahlung trifft senkrecht auf die Objekt- und Filmebene. In der vorliegenden Studie wurden die konventionellen Zahnfilmaufnahmen mit dem Gerät Heliodent MD (Sirona Dental Systems, Bensheim, Deutschland) hergestellt.Es wurde der Zahnfilm Kodak Insight IP 21, 30,5 x 40,5 mm (Carestream Health Inc, Rochester, NY, USA) benutzt. Die konventionellen Zahnfilmaufnahmen wurden von jedem der zehn Schweinekieferpräparate in folgender Weise durchgeführt: Der Zahnfilm wurde mit der zu belichtenden Seite nach oben auf eine plane Oberfläche gelegt und das jeweilige Präparat auf dem Film gerade positioniert, so dass Film und Präparat parallel im Strahlengang liegen. Der Tubus der Röntgenröhre wurde senkrecht über dem Präparat ausgerichtet, so dass die Winkelangabe am Tubus 0 Grad betrug. Die Aufnahme erfolgte somit in Rechtwinkeltechnik. Der Abstand zwischen Tubus und Präparat wurde für jedes Präparat mit $2 \mathrm{~cm}$ ausgemessen (Abb. 1a-c). Folgende Aufnahmeparameter wurden festgelegt: Röhrenspannung $60 \mathrm{kV}$, Röhrenstromstärke $7 \mathrm{~mA}$, Aufnahmezeit 0,12 Sek. Es erfolgte keine nachträgliche Bildbearbeitung. 


\begin{tabular}{|l|c|}
\hline Technische Daten & \\
\hline Röhrentyp & SR 60/70/7 \\
\hline Röhrenspannung & $\mathbf{6 0 ~ k V}$ \\
\hline Röhrenstromstärke & $\mathbf{7 ~ m A ~}$ \\
\hline Aufnahmezeit & $\mathbf{0 , 1 2}$ Sek. \\
\hline Filterung & $1,5 \mathrm{~mm} \mathrm{AL}$ \\
\hline Brennfleckgröße & $0,7 \mathrm{~mm}$ \\
\hline
\end{tabular}

Tabelle 2: Technische Daten des Heliodent MD (Sirona Dental Systems, Bensheim, Deutschland) Die fettgedruckten Aufnahmeeinstellungen entsprachen den Einstellungen in dieser Studie.
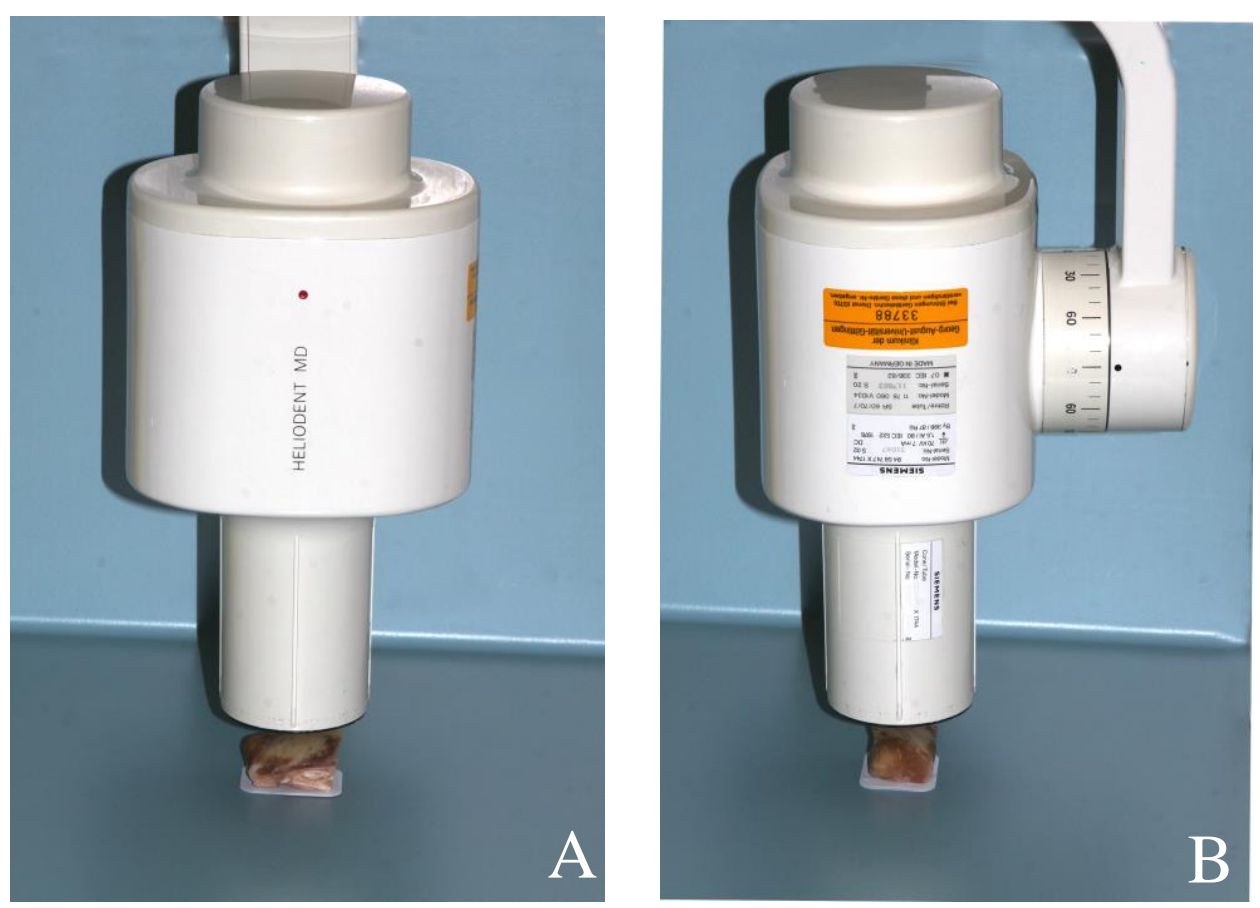

Abbildung 1: A und B: Versuchsaufbau zur Aufnahme eines konventionellen Zahnfilmes. Der Tubus ist senkrecht $\left(0^{\circ}\right)$ und zentral über dem Schweinekieferpräparat ausgerichtet. Das Präparat liegt zentral auf dem Film und ist parallel zu den Seitenkanten des Filmes positioniert. 


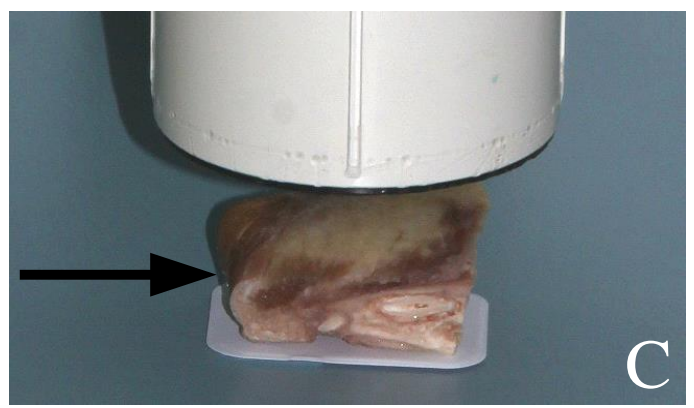

C: Bildausschnitt aus Abb. 1A; Position des Präparates auf dem Zahnfilm und unter dem Tubus. Der Pfeil zeigt auf den Alveolarkamm und die Position des Zahnimplantates.

\subsubsection{Digitale Zahnfilmaufnahmen}

Die Durchführung einer digitalen Zahnfilmaufnahme unterscheidet sich durch die Speicherart und die Entwicklung der Röntgenaufnahme von der konventionellen Zahnfilmaufnahme. Die Aufnahmetechnik, bei der Objekt und Film parallel liegen und der Zentralstrahl senkrecht auf diese Ebenen gerichtet ist, ist identisch. In der aktuellen Untersuchung wurden die digitalen Zahnfilmaufnahmen anhand einer Speicherfolie (2- black, Größe 2, 3 x 4 cm, Dürr Dental, Bietigheim-Bissingen, Deutschland) durchgeführt, die nach der Aufnahme durch ein passendes Scan-Gerät (Vista scan mini plus, Dürr Dental, Bietigheim-Bissingen, Deutschland) ausgelesen wird. Das aufgenommene Röntgenbild wird daraufhin in einer zum Röntgensystem passenden Software auf dem Computerbildschirm dargestellt. Die Software erlaubt es, durch Bearbeitungen zum Beispiel von Kontrast und Helligkeit das Bild zu verändern, Bildausschnitte zu vergrößern oder mit einer Messfunktion Strecken zwischen anatomisch bedeutenden Strukturen zu bestimmen. Die Aufnahmen wurden mit dem Gerät Miniray (SOREDEX, Helsinki, Finnland) durchgeführt. Die technischen Daten des Gerätes finden sich in Tab. 3. 


\begin{tabular}{|l|c|}
\hline Technische Daten & KL-21-SB \\
\hline Röhrentyp & $60-70 \mathrm{kV}(\mathbf{7 0} \mathbf{~ k V})$ \\
\hline Röhrenspannung & $\mathbf{7 ~ m A}$ \\
\hline Röhrenstromstärke & $\mathbf{0 , 1 2}$ Sek. \\
\hline Aufnahmezeit & $2 \mathrm{~mm} \mathrm{AL}$ \\
\hline Filterung & $0,7 \mathrm{~mm}$ \\
\hline Brennfleckgröße & 1 \\
\hline
\end{tabular}

Tabelle 3: Technische Daten des Miniray, SOREDEX, Helsinki, Finnland (in der Studie angewandte Parameter sind fett gedruckt)

Die digitalen Zahnfilmaufnahmen wurden unter den gleichen Voraussetzungen wie die konventionellen Aufnahmen hergestellt. Die jeweilige Schweinekieferprobe wurde auf der in eine Schutzfolie eingeschweißten Speicherfolie auf einer planen Oberfläche positioniert. Der Tubus der Röntgenröhre wurde mit $2 \mathrm{~cm}$ Abstand zur Probe ausgerichtet. Die rechteckige Tubusöffnung wurde genau über dem Präparat und der Speicherfolie positioniert (Abb. 2 AC). Dann erfolgte die Aufnahme mit einer Röhrenspannung von $70 \mathrm{kV}$ und einer Stromstärke von $7 \mathrm{~mA}$, bei einer Belichtungszeit von 0,12 Sek. 

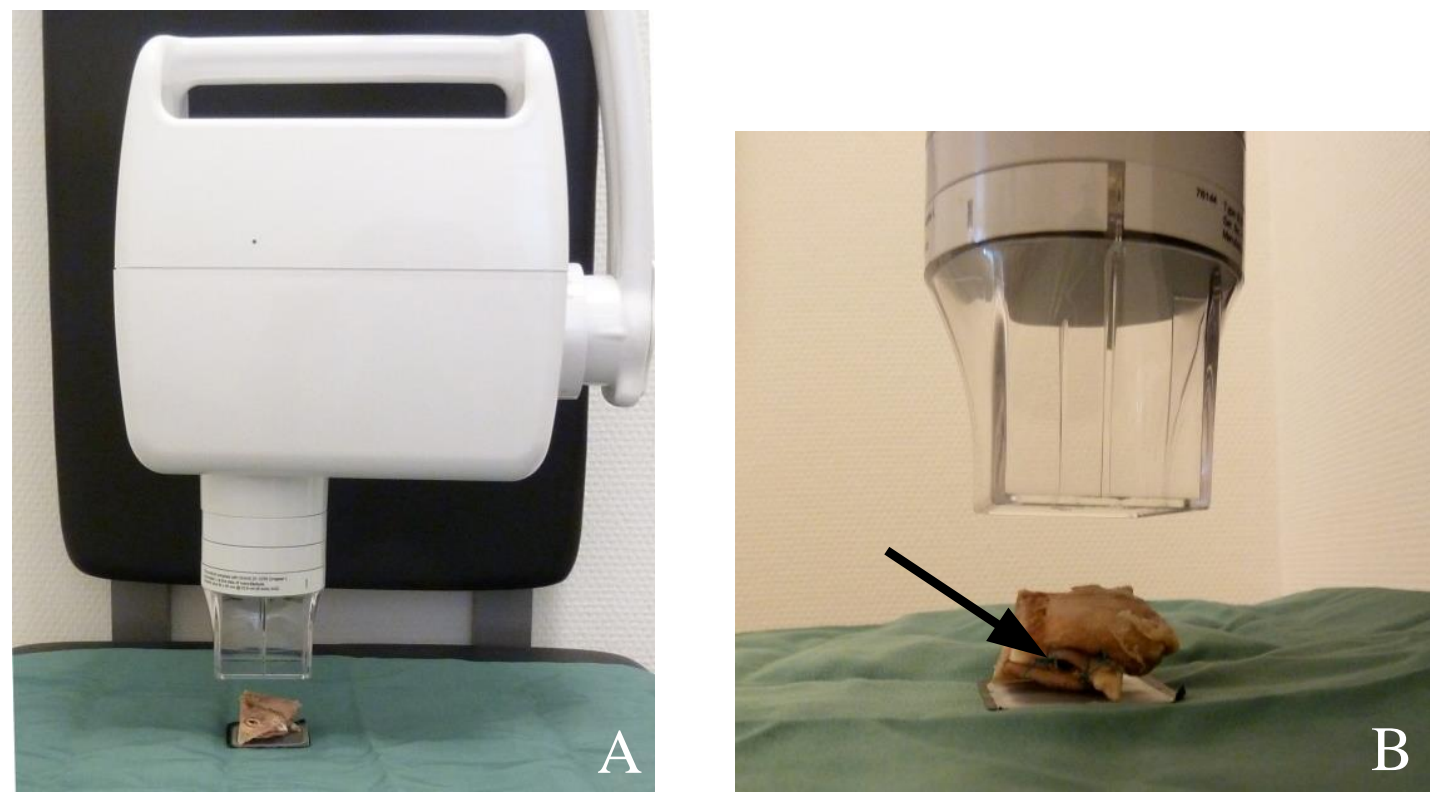

Abbildung 2: A und B: Versuchsaufbaus zur Aufnahme eines digitalen Zahnfilmes. Das Schweinekieferpräparat liegt auf der Speicherfolie und senkrecht darüber ist der rechteckige Tubus des digitalen Zahnfilmgerätes mit einem Abstand von $2 \mathrm{~cm}$ ausgerichtet. Der Pfeil in Abb. 2 B zeigt auf das in den Alveolarknochen inserierte dentale Implantat.

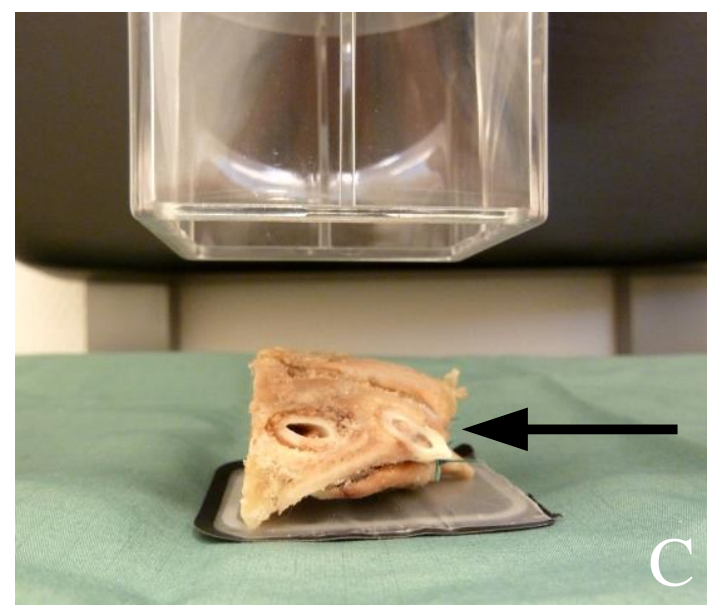

C: Darstellung derselben Position wie in Abb. 2 A. Dargestellt ist die Positionierung des

Schweinekieferpräparates auf der Speicherfolie von lateral. Der Tubus des digitalen Röntgengerätes ist senkrecht mit $2 \mathrm{~cm}$ Abstand über dem Präparat ausgerichtet. Der Pfeil zeigt auf den Alveolarkamm und die Einbringrichtung des dentalen Implantates. 


\subsubsection{Digitale Volumentomographie (DVT)}

Die digitale Volumentomographie ist eine dreidimensionale Röntgenmethode für den Kopfund Halsbereich.

In dieser Untersuchung wurde das DVT-Gerät PaX-Zenith 3D (Orangedental-VATECH Co, Ltd \& E-Woo, Hwaseong, Südkorea) verwendet. Die Projektionsaufnahmen, die während der Rotation um den Patientenkopf entstehen, werden von einem flatpanel detector mit CMOSSensor (complementary metal oxide semiconductor) aufgenommen und dann zu einem dreidimensionalen Datensatz umgerechnet. Die technischen Daten des Gerätes finden sich in Tabelle 4.

\begin{tabular}{|l|c|}
\hline Technische Daten & \\
\hline Aufnahmeverfahren & cone beam computed tomography \\
\hline Strahlerzeugung & $\begin{array}{c}\text { Kegelstrahl, Mikroprozessorgesteuerter } \\
\text { Hochfrequenzgenerator mit konstantem Potential }\end{array}$ \\
\hline Detektor & CMOS flatpanel (APS) \\
\hline Rotationsbereich & $360^{\circ}$ \\
\hline Anzahl Projektionen pro Umlauf & 375 / 600 \\
\hline Aufnahmevolumen (FOV) & sitzend \\
\hline Patientenposition & $50 \times 5$ mm Al \\
\hline Filterung & $0,5 \times 0,5$ mm \\
\hline Brennfleckgröße & $15-24$ Sek. \\
\hline Aufnahmezeit & $10-120$ Sek. \\
\hline Rekonstruktionsdauer & 14 Bit: 16384 \\
\hline Graustufen & \\
\hline Röntgenröhre & mm) \\
\hline Röhrentyp & $50-120 \mathrm{kV}$ (120 kV) \\
\hline Röhrenspannung & $-10 \mathrm{~mA}$ (6 mA) \\
\hline Röhrenstromstärke & \\
\hline
\end{tabular}

Tabelle 4: Technische Daten des PaX-Zenith 3D, Orangedental-Vatech, Südkorea (in der Studie angewandte Parameter sind fett gedruckt) 
Zur Aufnahme der DVT-Datensätze wurden die Schweinekieferpräparate auf der Kinnablage des DVT-Gerätes zentral und mit der vestibulären Seite des Schweinekiefers nach vorn positioniert. Dabei wurde die darzustellende Region „Mandibula zentral“ im SoftwareProgramm ausgewählt. Die Position des Präparates wurde mit Hilfe des Laservisiers in der Median-Sagittalebene eingestellt, so dass das Implantat zentral im Strahl des Laservisiers lag. Durch Anpassung der Kinnablagenhöhe wurde mit Hilfe eines weiteren Laservisiesr die zentrale, vertikale Position des Implantats in der interessierten Region (region of interest) festgelegt. Das Präparat war senkrecht zur Unterlage positioniert. Falls dies nicht möglich war, wurde eine senkrechte Position unter Zuhilfenahme von Periphery-Wachs hergestellt. Daraufhin wurde die Aufnahme durchgeführt (Abb. 3A-C). Die Aufnahmeparameter finden sich in Tabelle 5, der Versuchsaufbau ist in Abbildung 3 dargestellt.

\begin{tabular}{|l|c|}
\hline Aufnahmeparameter & dentaler DVT-Modus \\
\hline Aufnahmemodus & Mandibula zentral \\
\hline Aufnahmebereich & $80 \times 60 \mathrm{~mm}$ \\
\hline $\begin{array}{l}\text { Größe des Aufnahmefeldes } \\
\text { (FOV Modus) }\end{array}$ & $0,12 \mathrm{~mm}$ \\
\hline Auflösung (Voxelgröße) & 24 Sek. \\
\hline Aufnahmedauer (Abtastzeit) & Metallartefaktreduktion (MAR) \\
\hline Rekonstruktionsmodus & 42 Sek. \\
\hline Rekonstruktionszeit & . \\
\hline
\end{tabular}

Tabelle 5: Aufnahmeparameter des DVT-Gerätes PaX-Zenith 3D, Orangedental-Vatech, Südkorea. Das mittig positionierte Präparat wurde im Aufnahmebereich „Mandibula zentral“ aufgenommen, um eine möglichst eingegrenzte Aufnahmeregion für die Aufnahme zu erhalten. Das Aufnahmefeld entspricht dem zweitkleinsten möglichen Aufnahmefeld (80 x 60 mm) mit einer Auflösung von 0,12 mm. 

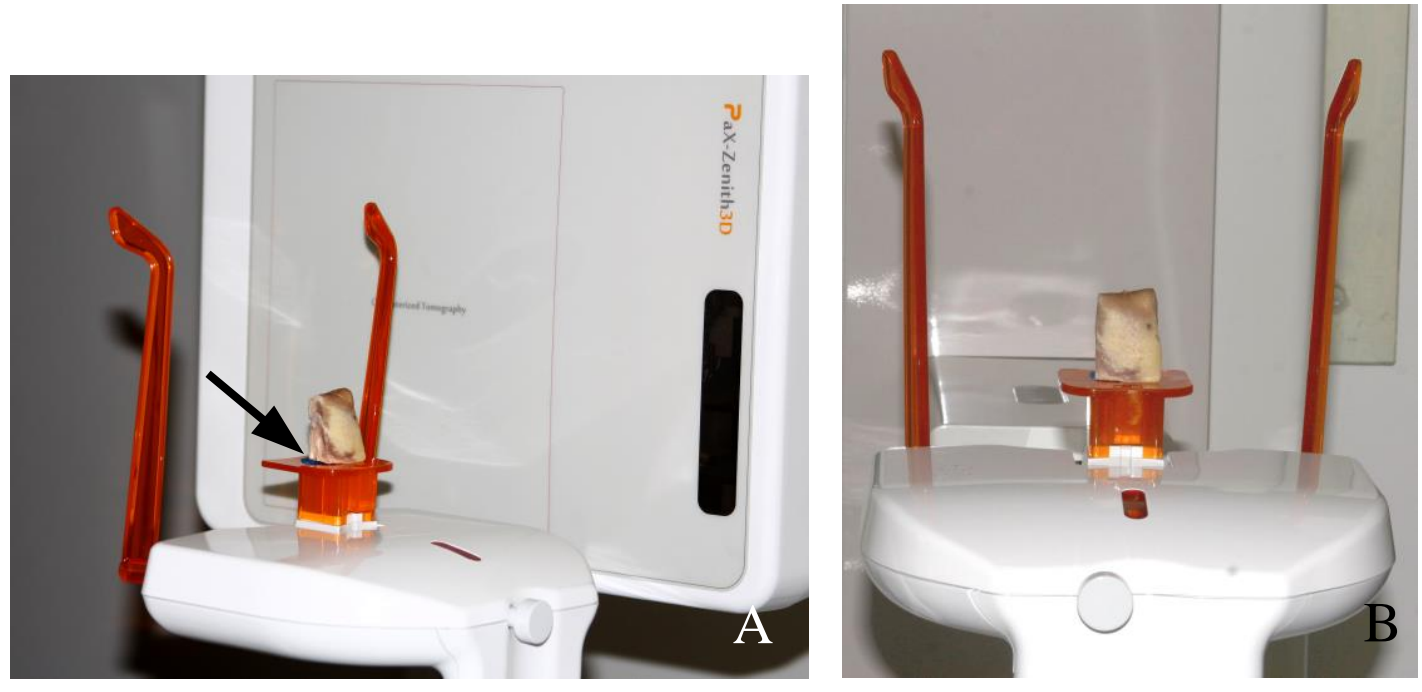

Abbildung 3: A und B: Versuchsaufbau DVT (PaX-Zenith 3D, Orangedental-Vatech, Südkorea). Dargestellt ist das Schweinekieferpräparat auf der Kinnstütze des DVT-Gerätes. Es ist zentral positioniert, sowie mit Hilfe von Periphery-Wachs senkrecht zur Unterlage ausgerichtet. Abb. A zeigt die senkrechte Positionierung durch das Periphery-Wachs (Pfeil), Abb. B zeigt das Präparat von der vestibulären Seite auf der Kinnablage.

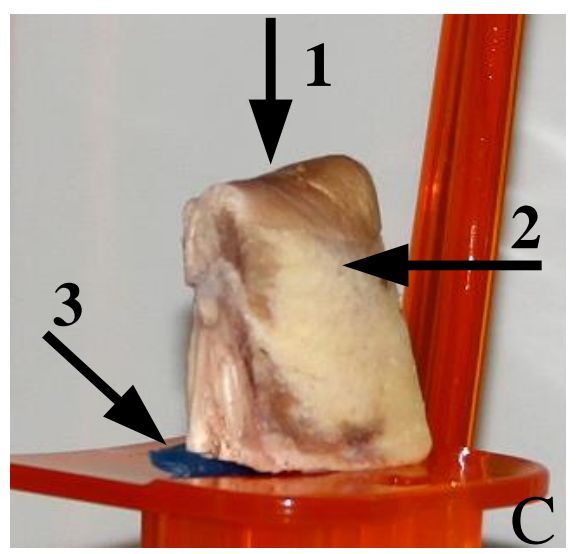

C: Bildausschnitt aus Abb. 3A; Vergrößerung zur verdeutlichten Darstellung der Position der Schweinekieferpräparates auf der Kinnablage. Pfeil 1 zeigt auf den Alveolarkamm und die Position des inserierten dentalen Implantates. Pfeil 2 bezeichnet die vestibuläre Fläche des Schweinekieferpräparates. Pfeil 3 deutet auf das Periphery-Wachs. 


\subsection{Randomisierte Evaluation durch zehn Untersucher}

Die drei röntgenologischen Verfahren konventioneller Zahnfilm, digitaler Zahnfilm und digitale Volumentomographie wurden anhand von jeweils zehn Aufnahmen in einer randomisierten Reihenfolge verglichen.

Dabei wurde die Darstellbarkeit von Knochengewebe und enossalen Implantaten in diesen drei Verfahren durch zehn Untersucher beurteilt.

\subsubsection{Untersucher}

Die Bilddaten wurden durch zehn Untersucher ausgewertet. Dabei handelte es sich um Studenten des klinischen zahnmedizinischen Studienabschnittes. Die Vorkenntnisse dieser Untersuchergruppe waren -auf die zweidimensionalen Röntgentechniken bezogen- fundiert, mit der Befundung von dreidimensionalen DVT-Aufnahmen waren alle Untersucher bisher nicht vertraut.

Vor der Datenevaluation besuchten die Untersucher einen sechsstündigen DVT-Kurs. In einem theoretischen Teil von ca. 90 min. wurden die Grundlagen der Funktionsweise eines DVT-Gerätes, zahnmedizinische Indikationen und Kontraindikationen sowie die Befundung des dreidimensionalen Datensatzes am Computer vorgestellt. Zudem wurden die einzelnen Funktionen der Bearbeitungssoftware erläutert. Im praktischen Teil des Kurses beurteilten die Untersucher mehrere anonymisierte Patientenfälle selbstständig an einem Computer. Nach Durchlauf dieses Kurses befundeten die Untersucher die Aufnahmen der vorliegenden Untersuchung. 


\subsubsection{Kalibrierung der Untersucher}

Vor Beginn der Datenauswertung wurden alle Untersucher durch eine Computerpräsentation mit dem Ablauf der Befundung sowie mit den einzelnen Schritten des Versuchsaufbaus vertraut gemacht.

Zunächst wurden die einzelnen möglichen Defekte im Alveolarknochen und an den Implantaten erläutert sowie die möglichen Fremdmaterialien vorgestellt. Dabei wurde ein Fremdmaterial (Fragment einer K-Feile) zusätzlich aufgezählt, das in den Schweinekieferpräparaten nicht vorhanden ist. Das Fragment einer K-Feile hat in einer röntgenologischen Darstellung viel Ähnlichkeit mit einem Guttaperchastift. Dies hatte den Zweck, dass die Untersucher nicht durch ein Ausschlussverfahren eine Fremdmaterialklassifizierung durchführen können. Sie mussten bestimmte Merkmale erkennen und diese richtig zuordnen. Für die Erfassung der metrischen Werte wurde den Untersuchern eine einheitliche Vorgehensweise zur Längen- und Durchmesserbestimmung der Implantate vorgegeben. Die Herstellerangaben der Implantatlängen und -durchmesser sowie die Durchmesser der Stahlkugeln in den Präparaten waren den Untersuchern nicht bekannt. Sie wurden lediglich informiert, dass sich die metrischen Werte der Implantate und Fremdkörper unterscheiden können. Im Anschluss wurden der Fragebogen und die randomisierte Abfolge der Befundung erläutert. Außerdem fand eine Einweisung in die Bearbeitungssoftware der digitalen Zahnfilmaufnahmen statt. 


\subsection{Prüfbogen}

Die Auswertung und Analyse der Bilddaten erfolgte anhand eines Fragenbogens (Abb. 4).

Der Fragebogen unterteilte sich in verschiedene Rubriken. Zunächst wurde nach dem Vorhandensein eines Implantates gefragt. Darauf folgend sollten die metrischen Werte Länge und Durchmesser des Implantates mit der vorgegebenen Messmethode bestimmt werden. Zudem sollte eine Aussage über die Differenzierbarkeit der Knochenstrukturen Spongiosa und Kompakta und der Zahnstrukturen Schmelz und Dentin getroffen werden. Die folgende Rubrik befasste sich mit dem Vorhandensein eines Knochendefektes. Eine positive Antwort ergaben nachfolgende Fragen zur dreidimensionalen Darstellung des Defektes, Klassifizierung des Defektes und zur Lokalisation im Präparat. Im nächsten Abschnitt erfolgte die Auswertung der Bilddaten hinsichtlich eines Implantatdefektes. War dieser vorhanden, folgten ebenfalls die Evaluation der Defektklasse, die Bestimmung der Lokalisation und die Entscheidung, ob eine dreidimensionale Darstellung des Defektes möglich ist. Im Abschnitt der Fremdkörperbestimmung musste zunächst die Klassifizierung nach Erkennen des Fremdkörpers durchgeführt werden. Daraufhin wurde die Anzahl der jeweiligen Fremdkörper bestimmt und deren Lokalisation ermittelt. Außerdem wurden bei Vorhandensein von Stahlkugeln und Guttaperchastiften der Durchmesser bzw. die Länge des Fremdkörpers gemessen. Im letzten Abschnitt wurden das Vorhandensein von Aufhärtungsartefakten und die Beeinträchtigung der Datenauswertung evaluiert. Schließlich hatte der Untersucher auch die Möglichkeit, weitere Befunde anzugeben. 
Auswertungsbogen: enossale Implantate in Schweinekiefern Randomisiert, kontrollierte Begutachtung der Präparate

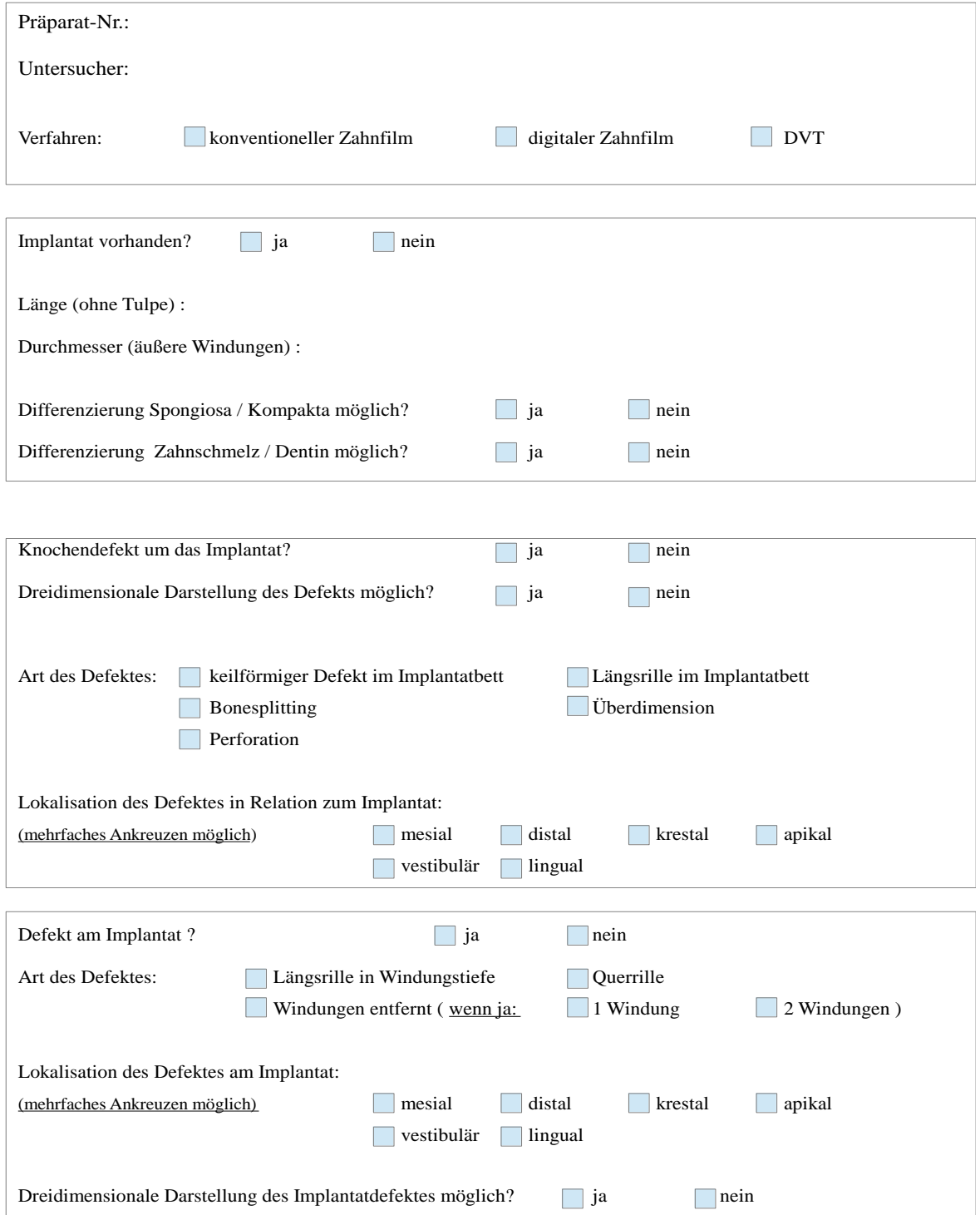




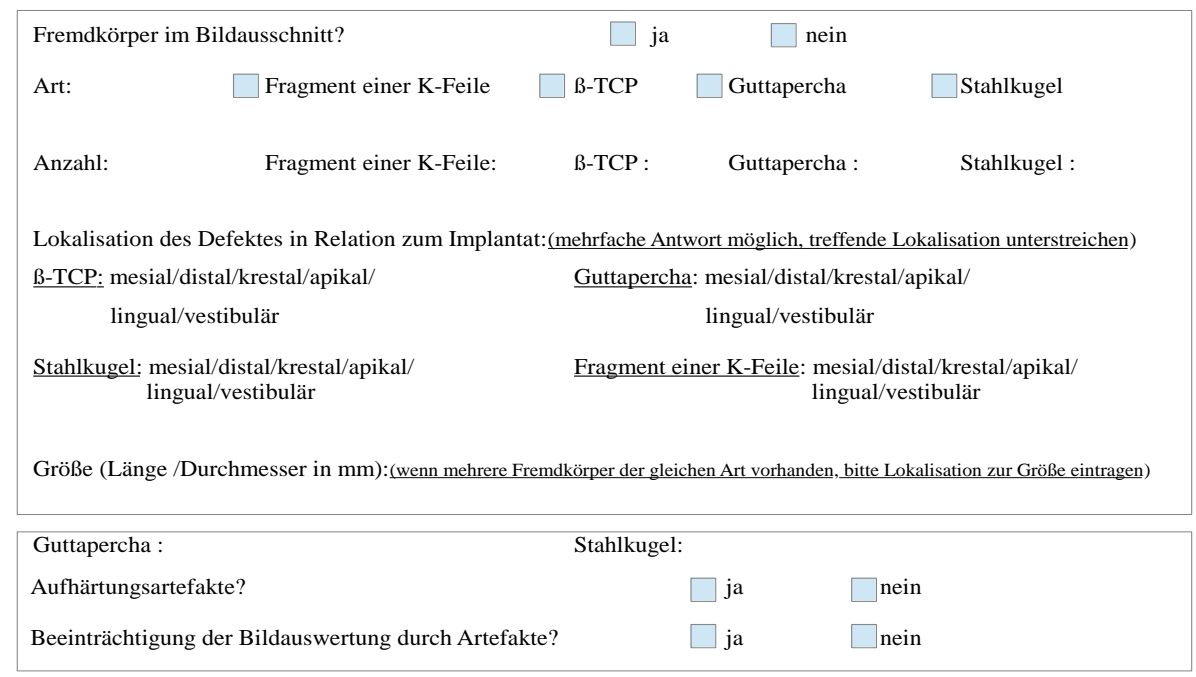

$\underline{\text { weitere Befunde? }}$

Abbildung 4: Prüfbogen zur Evaluation der Bilddaten

\subsection{Randomisierung der Datensätze}

Die zehn Schweinekieferpräparate wurden in jede der drei Röntgenverfahren aufgenommen.

Ein mögliches Wiedererkennen der Präparate wurde durch eine randomisierte Reihenfolge der Präparate erschwert. Dabei wurden die Präparate von den Untersuchern jedes Mal in einer unterschiedlichen Reihenfolge betrachtet. Außerdem wurde als erstes eine zweidimensionale Methode untersucht, danach folgte die dreidimensionale Methode und schließlich wurde die zweite zweidimensionale Methode betrachtet. Hiermit sollte ein Wiedererkennen der Zahnfilmaufnahmen verringert werden. Die Randomisierung der Präparate wurde innerhalb einer Methode mit dem Programm „R“ (Permutation ohne Wiederholung) durchgeführt (Medizinische Statistik der Universität Göttingen). Für alle Untersucher lagen die gleichen Bedingungen bei den Auswertungen vor. 


\subsection{Durchführung der Evaluation}

Die Auswertung der Datensätze wurde an fünf unterschiedlichen Tagen durchgeführt. Dabei werteten jeweils zwei Untersucher pro Tag alle Daten anhand der Fragebögen aus.

Jeder Untersucher erhielt mit den zehn Fragebögen pro Methode eine Vorlage mit der randomisierten Reihenfolge der Präparate. Nach Betrachtung der Aufnahmen in einer Methode wurden die ausgefüllten Fragebögen mit den randomisierten Listen eingesammelt und ein neuer Satz für die folgende Methode ausgeteilt. Die Beurteilungen der digitalen Bilddaten fanden immer an den gleichen Computerbildschirmen der Firma Dell (P1913s, 19 Zoll; Dell GmbH, Frankfurt am Main, Deutschland) mit einer Auflösung von 1280 x 1024 Pixel statt.

\subsection{Statistische Auswertung}

Zunächst wurden die Messabweichungen für Implantatlänge und -durchmesser in den drei zu vergleichenden Methoden gegenüber gestellt. Anhand eines F-Tests (ANOVA, STATISTICA, StatSoft Europe) wurde eine statistische Signifikanz zwischen den drei Röntgenmethoden getestet.

Die erkannten Knochendefekte, Implantatdefekte und Fremdkörper wurden für jede Methode ausgezählt und prozentual graphisch dargestellt sowie eine Signifikanz zwischen den Methoden getestet (Chi-Quadrat-Test, STATISTICA, StatSoft Europe). Folgend wurde davon differenziert betrachtet, wie oft ein Defekt oder Fremdkörper bei Erkennen zusätzlich korrekt klassifiziert wurde. Anhand einer Kontingenztafel wurde für jede Methode ausgezählt, wie häufig ein Defekt/Fremdkörper korrekt oder falsch klassifiziert wurde. Die Verteilungen der korrekten und inkorrekten Klassifizierungen wurden dann auf einen signifikanten Unterschied zwischen den Methoden getestet (Fisher's exact test, 3 x 2 Kreuztabellen, vassarstats.net). Die Ergebnisse wurden außerdem für einen Vergleich zwischen zweidimensionalen und dreidimensionalen Verfahren ausgewertet und graphisch dargestellt. Schließlich wurde ein Vergleich innerhalb der Untersuchergruppe durchgeführt. Dabei wurde getestet, ob sich die Untersucher hinsichtlich ihrer Leistung in der Evaluation unterschieden. Dabei wurden die 
Ergebnisse der metrischen Werte (Vermessung der Implantate), die korrekte Befundung der Knochen- und Implantatdefekte und die korrekte Klassifizierung der Fremdkörper auf statistische Signifikanz getestet (metrische Werte F-Test, ANOVA; Defekte und Fremdkörper Chi-Quadrat-Test, STATISTICA, StatSoft Europe). Das Signifikanzniveau wurde für alle Untersuchungen auf $\mathrm{p}<0,05$ festgelegt. Die einzelnen Messungen und Ergebnisse wurden ausgewertet und graphisch in Boxplots oder als Säulendiagrammen dargestellt. 


\section{$\underline{\text { 3. Ergebnisse }}$}

\subsection{Bildbeispiele zur Darstellbarkeit in den unterschiedlichen}

\section{radiologischen Verfahren}

Die Auswertung der radiologischen Aufnahmen der verschiedenen Schweinekieferpräparate in dieser Untersuchung war in jeder der drei untersuchten Methoden möglich.

Unterschiedlich beschaffene Nachbargewebe, wie Kompakta und Spongiosa des Knochens sowie die einzelnen Strukturen der Zähne in Nachbarschaft der inserierten Implantate konnten beurteilt werden (Abb. 5, Abb. 6).
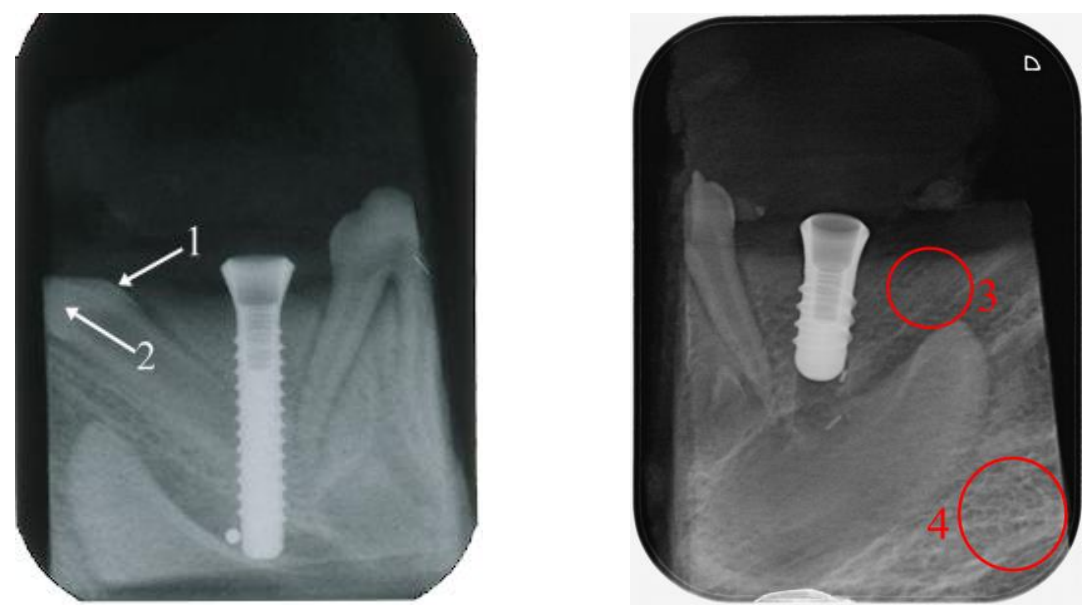

Abbildung 5: Differenzierung der Nachbargewebe, links konventioneller Zahnfilm (Pfeil 1: Zahnschmelz, Pfeil 2: Zahndentin), rechts digitaler Zahnfilm (3: Knochenkompakta, 4: Knochenspongiosa) 

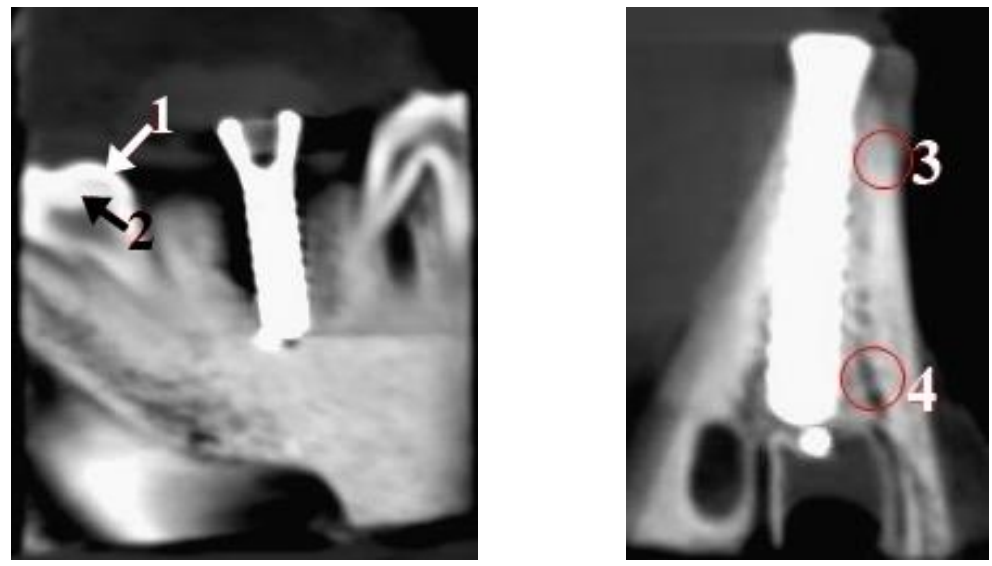

Abbildung 6: Differenzierung der Nachbargewebe in Ausschnitten des DVT: links koronaler Schnitt, Pfeil 1: Zahnschmelz, rechts sagittaler Schnitt, Pfeil 2: Zahndentin, 3: Knochenkompakta, 4: Knochenspongiosa

Die Manupulationen am Alveolarknochen und an den Implantaten konnten in den drei radiologischen Verfahren in unterschiedlicher Quantität und Qualität beurteilt werden. Die Defekte des Alveolarknochens wurden in den röntgenologischen Aufnahmen häufiger erkannt als die Implantatdefekte.
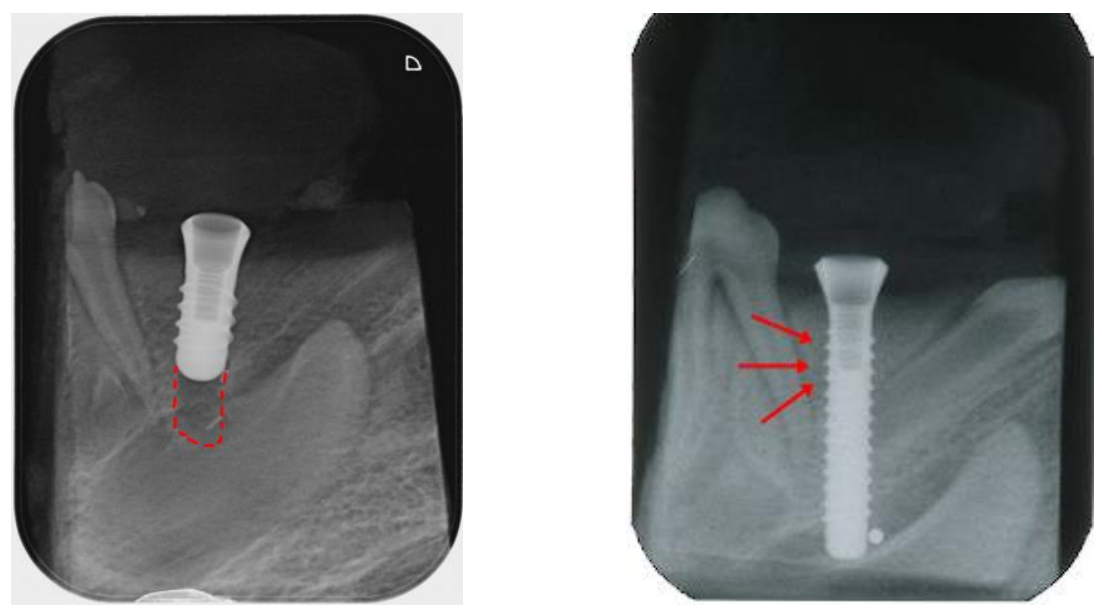

Abbildung 7: Knochendefekte in den zweidimensionalen Röntgenaufnahmen:

links (dig. ZF) Knochendefekt „Überdimension der Kavität“ in Präparat 13 (siehe gestrichelte Umrandung), rechts (konv. ZF) Knochendefekt A (1 mm tiefe Bohrung in $4 \mathrm{~mm}$ Höhe) in Präparat 12 (siehe Pfeile) 

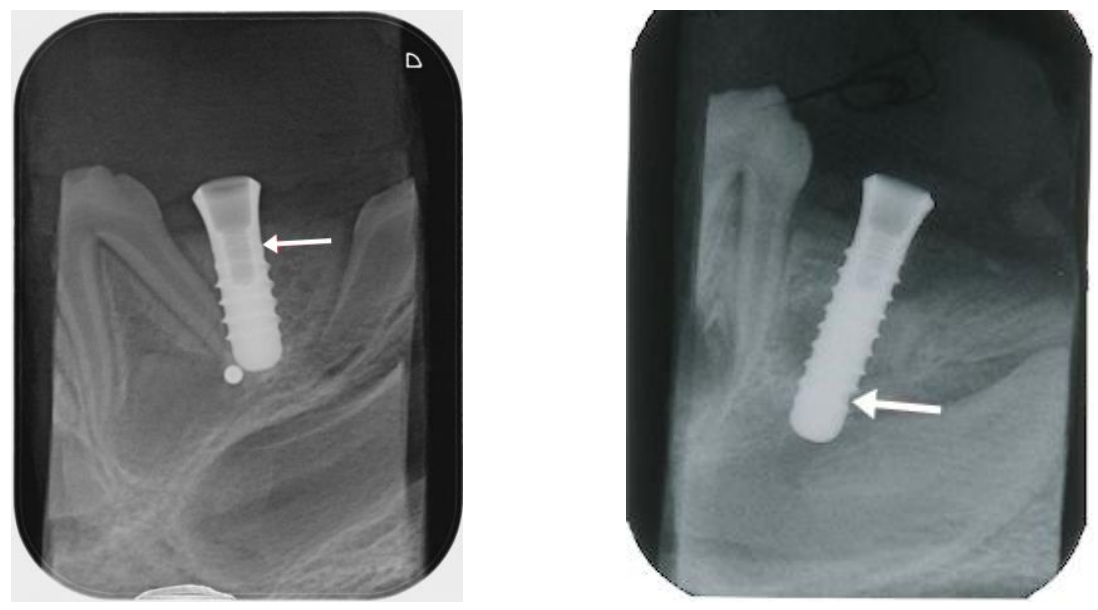

Abbildung 8: Implantatdefekt in den zweidimensionalen Röntgenaufnahmen: links (dig. ZF) Implantatdefekt B (obere Windung planiert) in Präparat 3 (siehe Pfeil), rechts (konv. ZF) Implantatdefekt C (Querrille in 0,5 mm tief) in Präparat 8
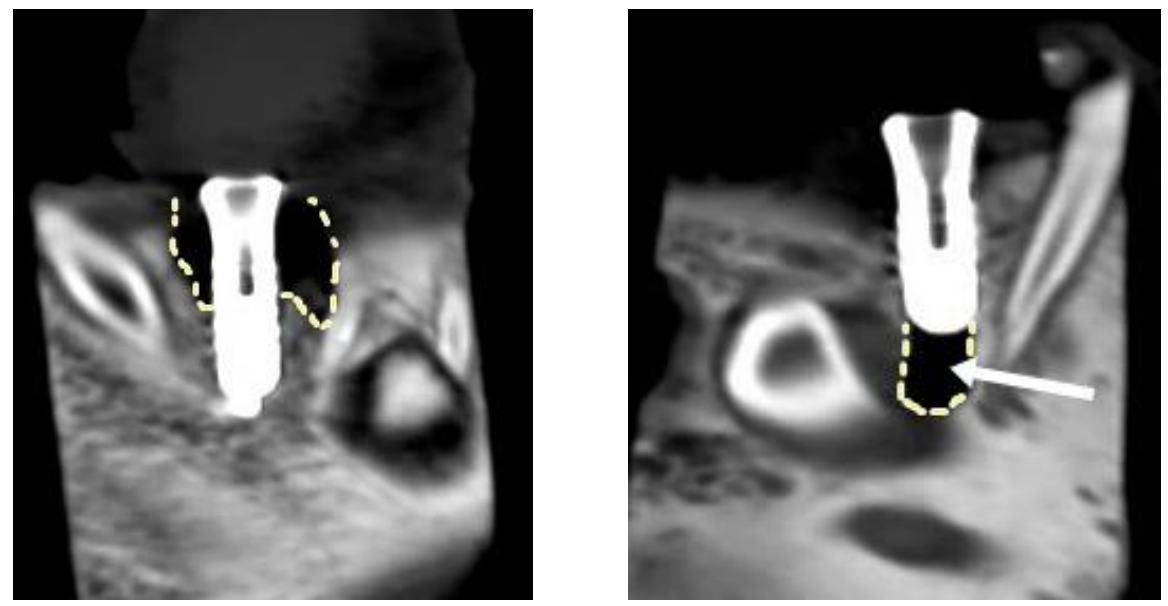

Abbildung 9: Alveolardefekte in DVT-Ausschnitten: links Knochendefekt „Bonesplitting“in Präparat 10 (siehe gestrichelte Umrandung), rechts Knochendefekt „Überdimension“ in Präparat 13 (siehe gestrichelte Umrandung und Pfeil)

In den DVT-Aufnahmen ergab sich durch Artefaktbildung im Aufnahmeprozess eine erschwerte Beurteilung der Implantate, der metallischen Fremdmaterialien und deren direkter Umgebung. Somit war die Evaluation dieser Körper in Hinsicht auf Defekte in den DVTAufnahmen eingeschränkt (Abb. 10). 

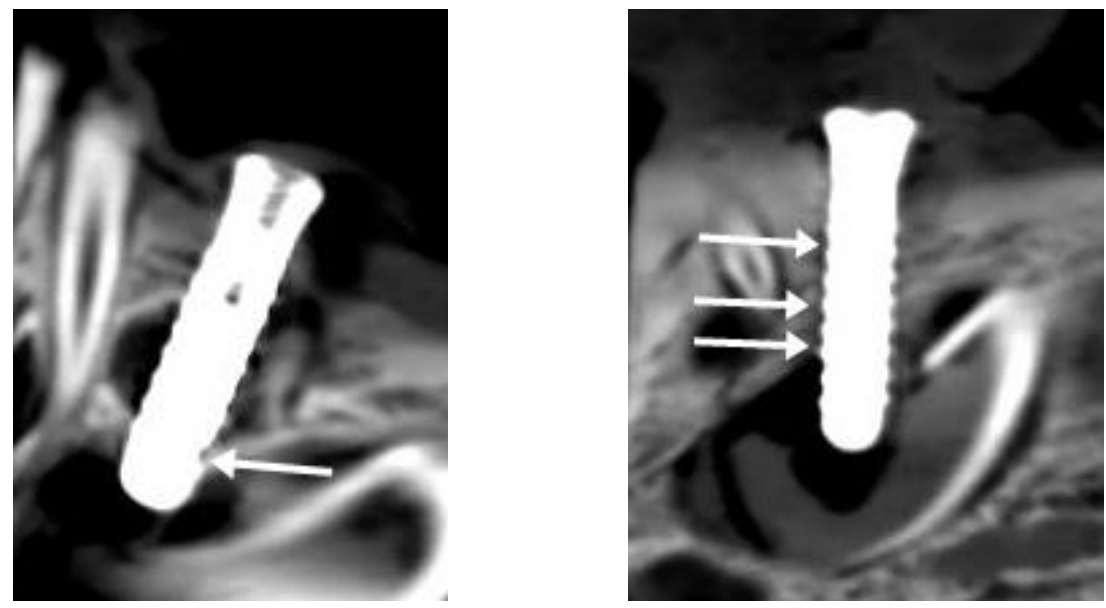

Abbildung 10: Implantatdefekte in DVT-Ausschnitten: links Implantatdefekt C (Querrille 0,5mm tief) in Präparat 8 (siehe Pfeil), rechts Implantatdefekt A (Längsrille in Windungstiefe) in Präparat 5 (siehe Pfeile)

Abhängig von der Darstellung in den Aufnahmen konnten Fremdmaterialen unterschiedlicher Dichte und Röntgenopazität erkannt und zugeordnet werden. Die Anwesenheit von Metallartefakten bedingte eine erschwerte Beurteilbarkeit in den dreidimensionalen DVTAufnahmen (Abb. 10). Die überlagerungsfreie Darstellung der einzelnen Strukturen in den Präparaten ließ dennoch eine Beurteilung in allen drei Raumebenen zu (Abb. 12). Die Limitation durch überlagerte Strukturen im Strahlengang grenzte die Evaluation der zweidimensionalen Aufnahmen ein (Abb. 11C).
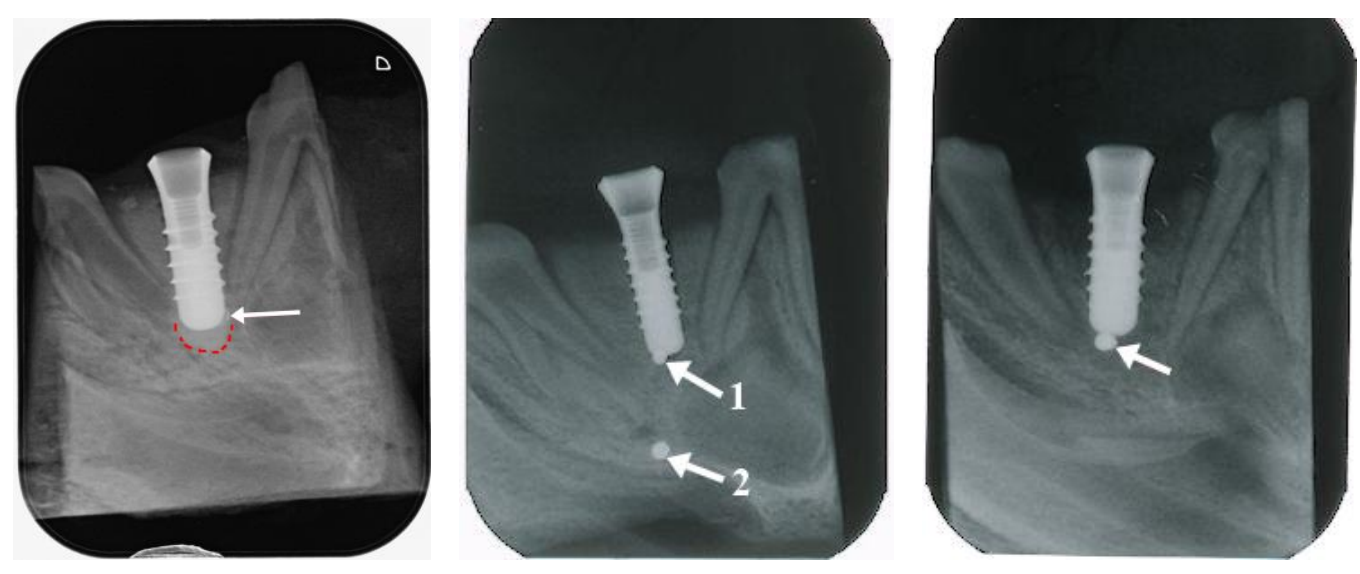

Abbildung 11: Fremdmaterialien in den zweidimensionalen Zahnfilmaufnahmen: A: dig. ZF; gestrichelte Umrandung begrenzt das Knochenersatzmaterial $\beta$-TCP und Pfeil zeigt auf Guttaperchastift in Präparat 12 B: konv. ZF; zwei Stahlkugeln in Präparat 14 (Kugeldurchmesser Pfeil 1: $1 \mathrm{~mm}$, Pfeil 2: 1,6 mm) C: konv. ZF; zwei sich überlagernde Stahlkugel in Präparat 10, siehe Pfeil (Durchmesser $1 \mathrm{~mm}$ und 1,6 mm) 

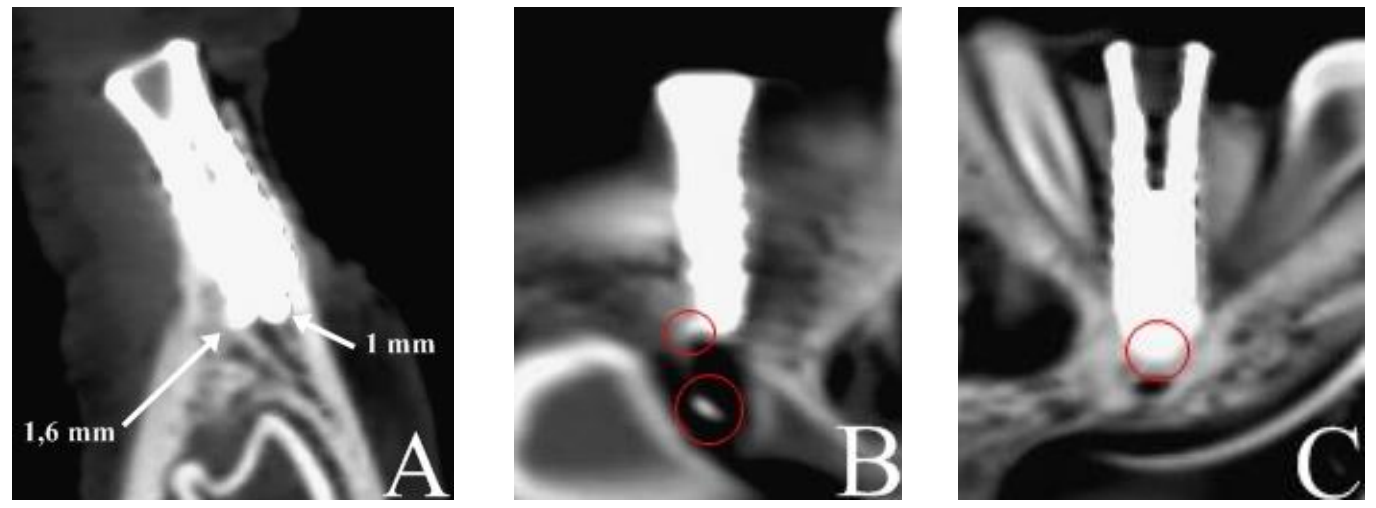

Abbildung 12: Fremdmaterialien in DVT-Ausschnitten: A: zwei Stahlkugeln in Präparat 10 (sagittaler Schnitt) B: zwei Guttaperchastifte in Präparat 13 (koronaler Schnitt, siehe rote Umrandung) C: B-TCP in Präparat 12 (koronaler Schnitt, siehe rote Umrandung)

Die Ermittlung der Implantatlängen und -durchmesser war mit jeder der drei röntgenologischen Aufnahmetechniken möglich. In den konventionellen Zahnfilmaufnahmen wurden die metrischen Werte anhand einer Messlehre ermittelt. Die entsprechende Software der digitalen Zahnfilmaufnahmen (Byzz, Orangedental) und der digitalen Volumentomographie (Ez3D 2009, Orangedental) ermöglichte in diesen beiden Techniken eine digitale Messung der Implantatlängen und -durchmesser (Abb. 13).
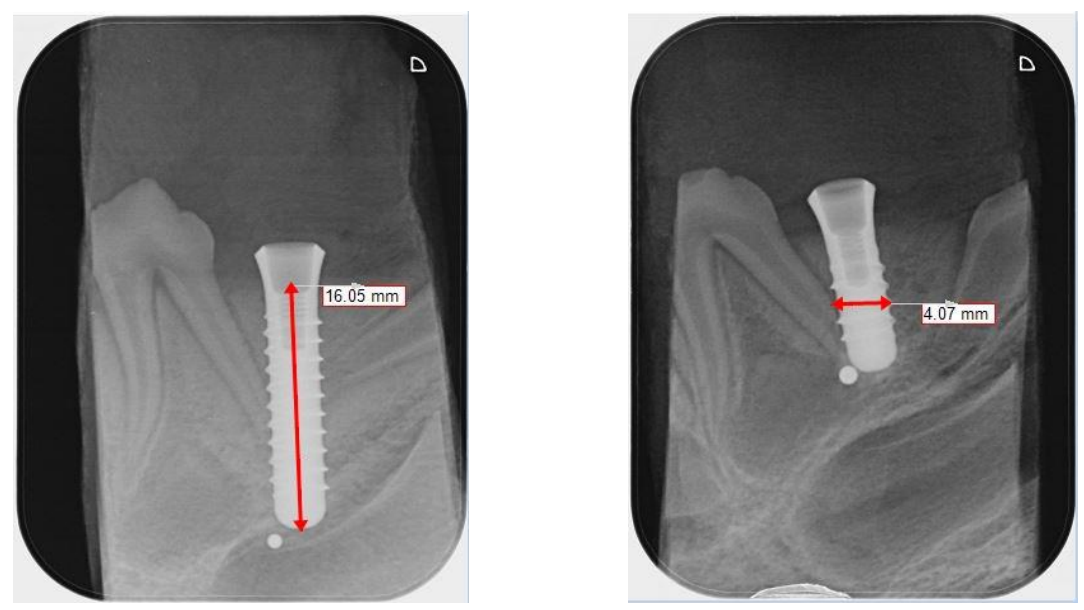

Abbildung 13: Messung der Implatatlänge (links) im Präparat 4 und des Implantatdurchmessers (rechts) im Präparat 3 in der digitalen Zahnfilmaufnahme (Software Byzz, Orangedental) 

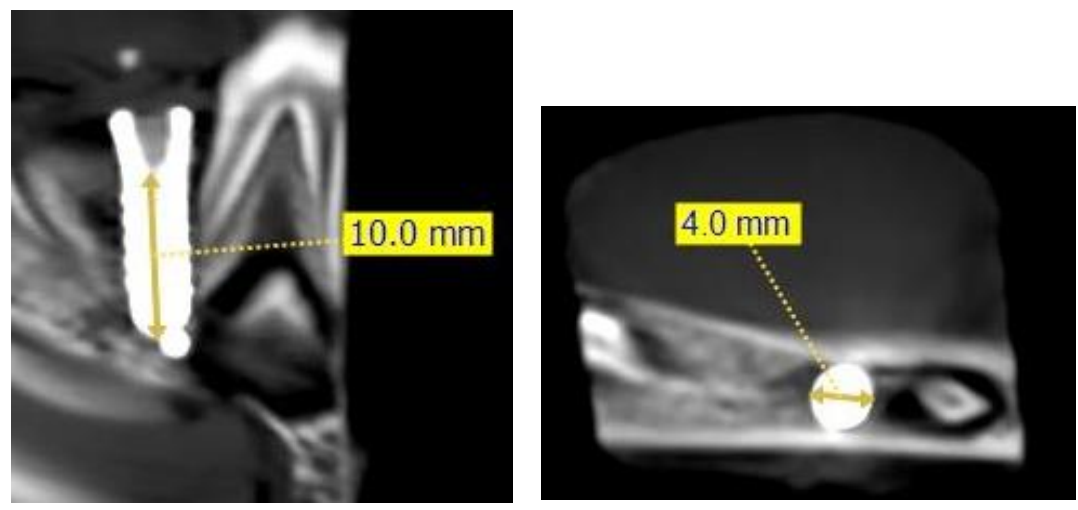

Abbildung 14: Messung der Implatatlänge (links) und des Implantatdurchmessers (rechts) im Präparat 11 in der DVT-Aufnahme (Ez3D 2009, Orangedental) 


\subsection{Der Vergleich der radiologischen Verfahren}

\subsubsection{Differenzierung der Nachbargewebe}

Eine Differenzierung der Nachbargewebe war in allen Methoden möglich. Dabei unterschied sich die Differenzierbarkeit von Kompakta und Spongiosa in den zweidimensionalen deutlich von der dreidimensionalen Methode. Bei der Befundung der konventionellen Zahnfilmaufnahmen war in 11 der Aufnahmen eine exakte Unterscheidung der beiden Gewebestrukturen erkennbar. In den digitalen Zahnfilmaufnahmen waren es $22 \%$. In der DVT konnte in $80 \%$ der Aufnahmen die Spongiosa von der Kompakta differenziert werden.

Die Differenzierung von Schmelz und Dentin war in den dreidimensionalen DVT-Aufnahmen in $39 \%$ der Aufnahmen möglich, in den zweidimensionalen Aufnahmen der digitalen Zahnfilmaufnahmen in $75 \%$ und in den konventionellen Zahnfilmaufnahmen in $53 \%$.

\subsubsection{Längenmessung der Implantate}

Mit allen röntgenologischen Verfahren konnte eine Messung aller Implantate durchgeführt werden. Die Messung der Implantatlängen wurde in den konventionellen Zahnfilmaufnahmen anhand einer Messlehre und in den digitalen Verfahren anhand einer Software durchgeführt:

- digitaler Zahnfilm: Byzz (Orangedental, Deutschland)

- DVT: Ez3D 2009 (Orangedental, Deutschland).

Die Präzision der drei röntgenologischen Verfahren, bezogen auf die Messabweichung der Herstellerangabe, unterschied sich signifikant voneinander ( $\mathrm{p}<0,0001$, F-Test) (Abb. 15). 


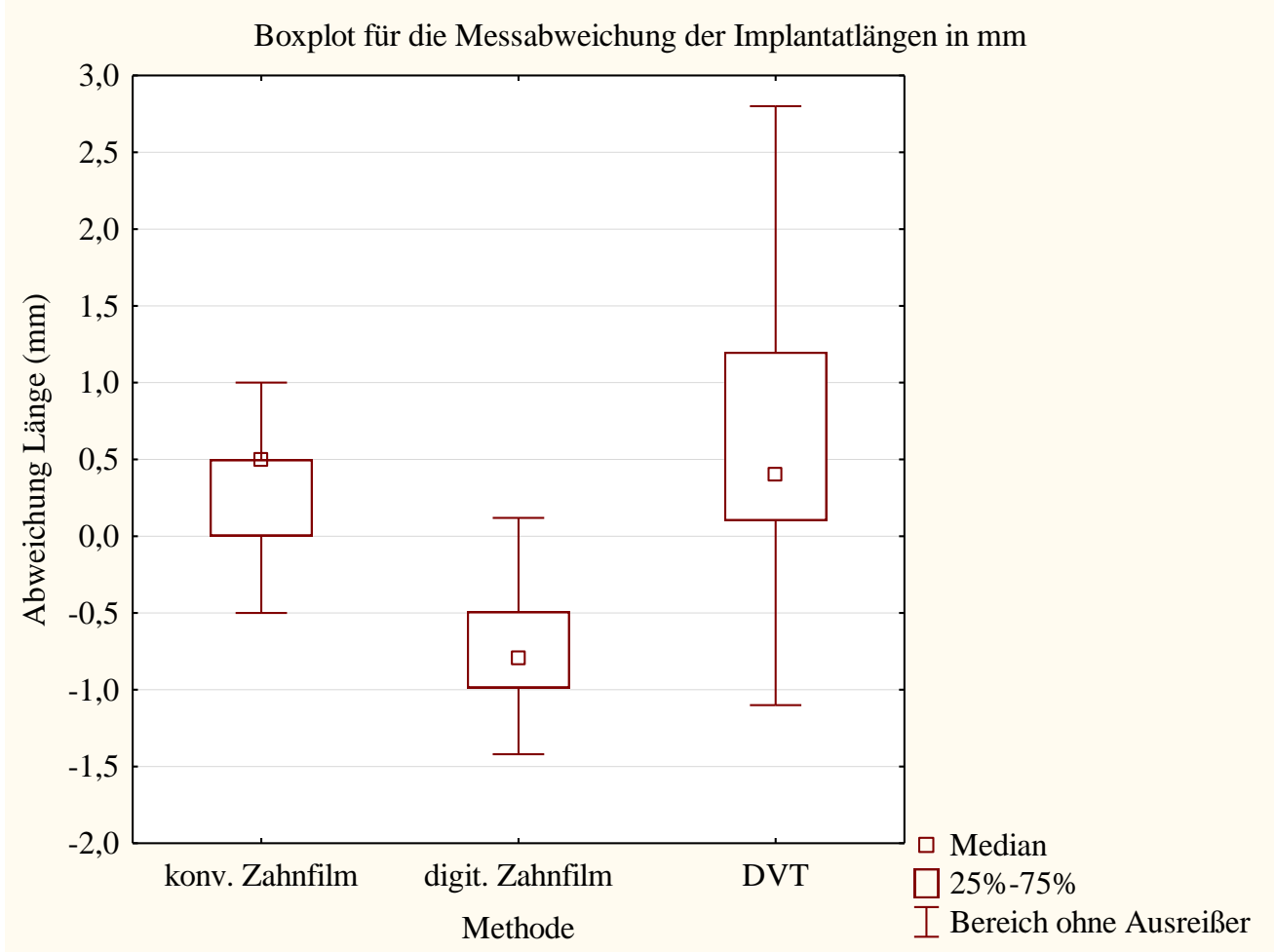

Abbildung 15: Dargestellt ist die absolute Messabweichung der Implantatlängen in mm. Die Messabweichungen werden unterteilt für die drei unterschiedlichen Röntgenverfahren konventioneller Zahnfilm, digitaler Zahnfilm und digitale Volumentomographie (DVT) abgebildet. Die Null-Linie entspricht in dieser Abbildung der Herstellerangabe der Implantatlänge. Die Boxen, Mediane und Whisker zeigen die Verteilung der Messabweichungen von der Herstellerangabe.

Bei der Messung der Implantate wurden nur Messwerte als korrekt anerkannt, die der Herstellerangabe mit einer Abweichung von $\pm 0,1 \mathrm{~mm}$ entsprachen. Die Messungen in den digitalen Zahnfilmaufnahmen entsprachen immer einer höheren Abweichung als in der oben genannten Bedingung. Daher wurde in dieser Methode keine Messung als korrekt anerkannt. In den konventionellen Zahnfilmaufnahmen wurden die Implantatlängen in $36 \%$ korrekt gemessen, in den DVT-Aufnahmen waren 20 \% der Messungen korrekt (Tab. 6; Abb. 16). 


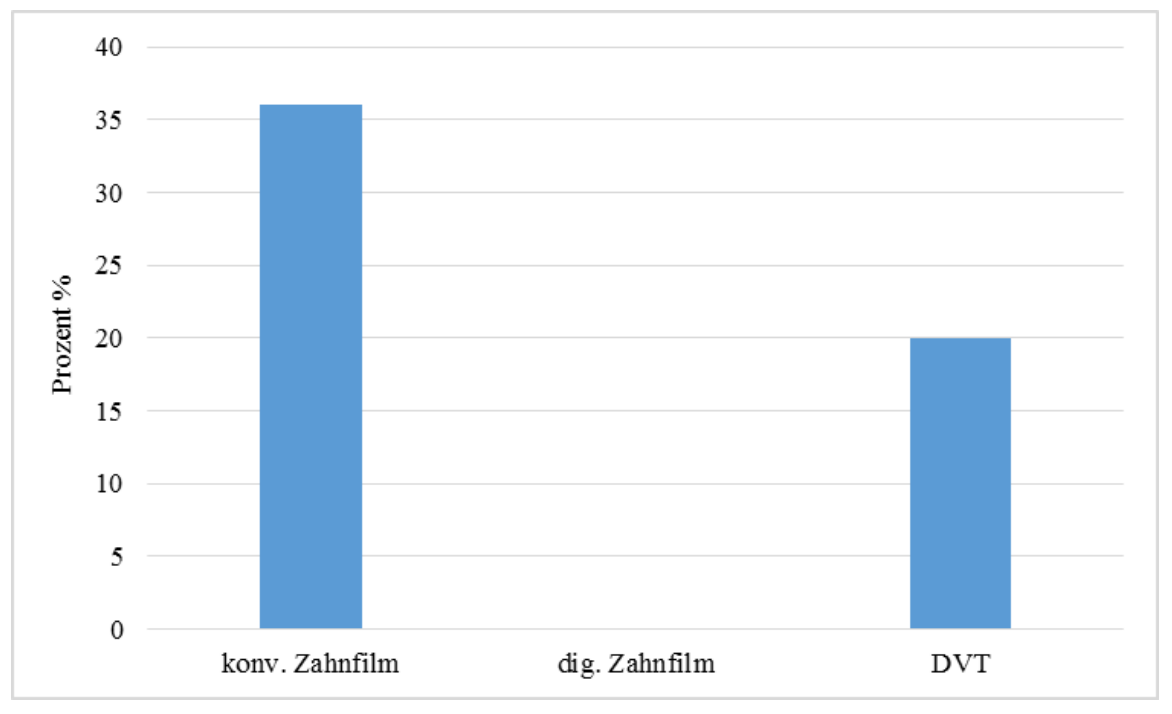

Abbildung 16: Implantatlängen: Darstellung des prozentualen Anteils der korrekt gemessenen Implantatlängen (inkl. Abweichung $+/-0,1 \mathrm{~mm}$ ) in den drei radiologischen Verfahren

\begin{tabular}{|l|l|}
\hline Röntgenologisches Verfahren & $\begin{array}{l}\text { Messabweichung bei der Längenbestimmung der zehn } \\
\text { Implantate (Mittelwerte in mm) }\end{array}$ \\
\hline konventioneller Zahnfilm & $+0,43$ (Standardfehler $\pm 0,12)$ \\
\hline digitaler Zahnfilm & $-0,61$ (Standardfehler $\pm 0,01)$ \\
\hline digitale Volumentomographie (DVT) & $+0,61$ (Standardfehler $\pm 0,15)$ \\
\hline
\end{tabular}

Tabelle 6: Bei der Messung der Implantatlängen wurden in den drei Methoden unterschiedliche Mittelwerte der Messabweichungen ermittelt. Sie werden für jede röntgenologische Methode aufgeführt, in Klammern befindet sich der jeweilige Standardfehler. Eine graphische Darstellung zu dieser Tabelle folgt in Abb. 17. 


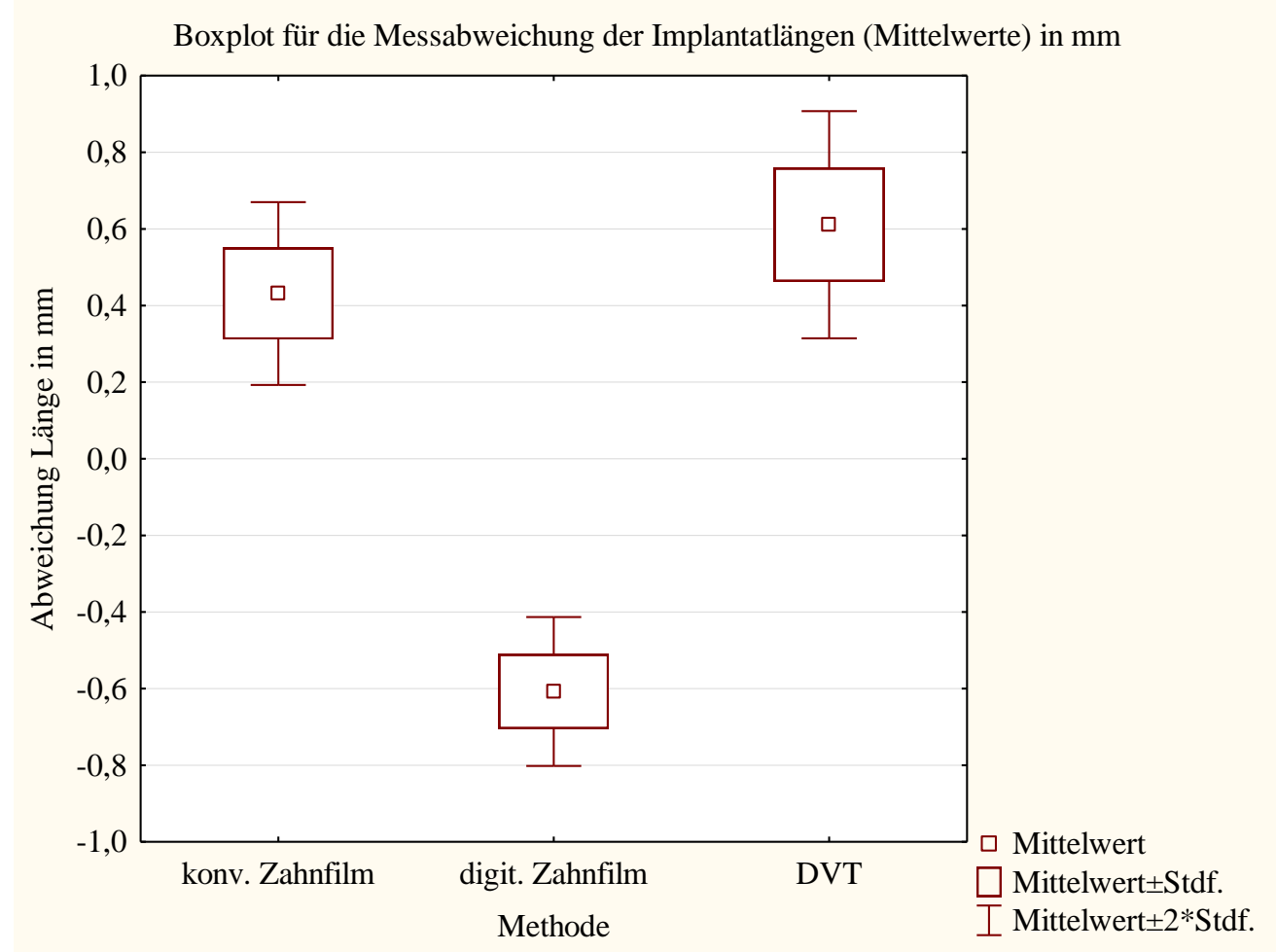

Abbildung 17: Dargestellt sind die Mittelwerte der Messabweichungen. Die Mittelwerte und Standardfehler unterscheiden sich in den drei Methoden. In dieser Grafik werden sie einander gegenüber gestellt. Die Null-Linie entspricht der Herstellerangabe der Implantatlängen. Für jede Röntgenmethode zeigen die Mittelwerte, Boxen und Whisker die Verteilung der Messabweichungen von der Herstellerangabe. Die Mittelwerte und Standardfehler sind Tab. 6 zu entnehmen.

\subsubsection{Durchmesserbestimmung der Implantate}

Die Bestimmung der Implantatdurchmesser war mit allen drei bildgebenden Verfahren durchführbar. Die beiden zweidimensionalen Methoden und die dreidimensionale digitale Volumentomographie (DVT) unterschieden sich in der Messgenauigkeit der Implantatdurchmesser signifikant voneinander ( $\mathrm{p}<0,0001$, F-Test) (Abb. 18). 


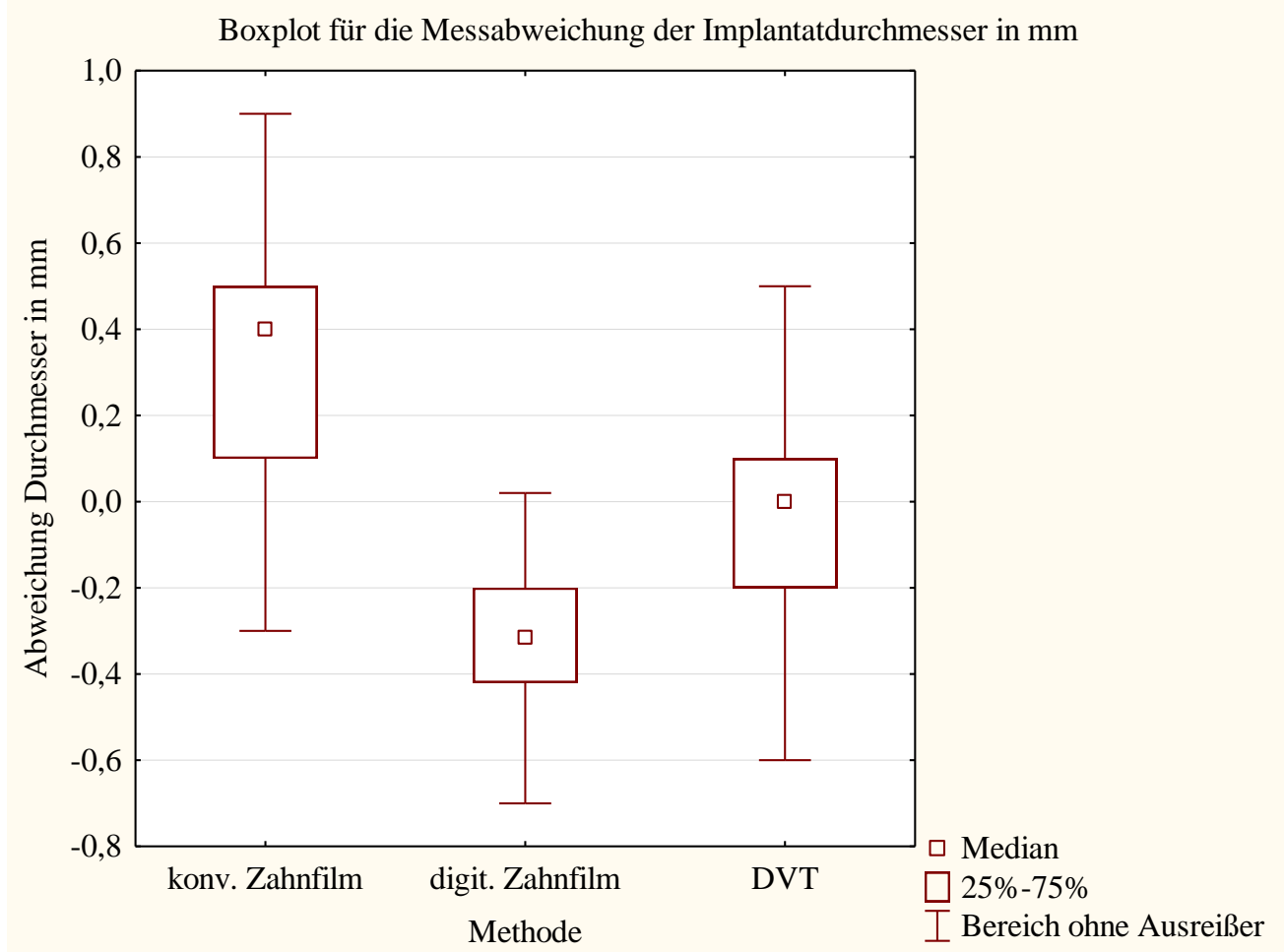

Abbildung 18: Dargestellt wird die absolute Messabweichung der Implantatdurchmesser in $\mathrm{mm}$. Die Abweichungen werden gesondert für jede röngtenologische Methode (konv. Zahnfilm, digit. Zahnfilm, DVT) abgebildet. Die Null-Linie stellt die Herstellerangabe des Implantatdurchmessers dar. Der Median, die Box und die Whisker zeigen die verschiedenen Messabweichungen in jeder Methode.

Bei der Durchmesserbestimmung konnten unterschiedliche Häufigkeiten von korrekten Messungen (Herstellerangabe $\pm 0,1 \mathrm{~mm}$ ) mit den drei unterschiedlichen Röntgenmethoden erzielt werden. Mit der digitalen Volumentomographie (DVT) wurden mehr als die Hälfte (53\%) der Durchmesser korrekt bestimmt. In den konventionellen Zahnfilmaufnahmen wurden $21 \%$ der Durchmesser korrekt bestimmt, in den digitalen Zahnfilmaufnahmen wurden $7 \%$ korrekt beurteilt (Tab. 7; Abb. 19, Abb. 20). 


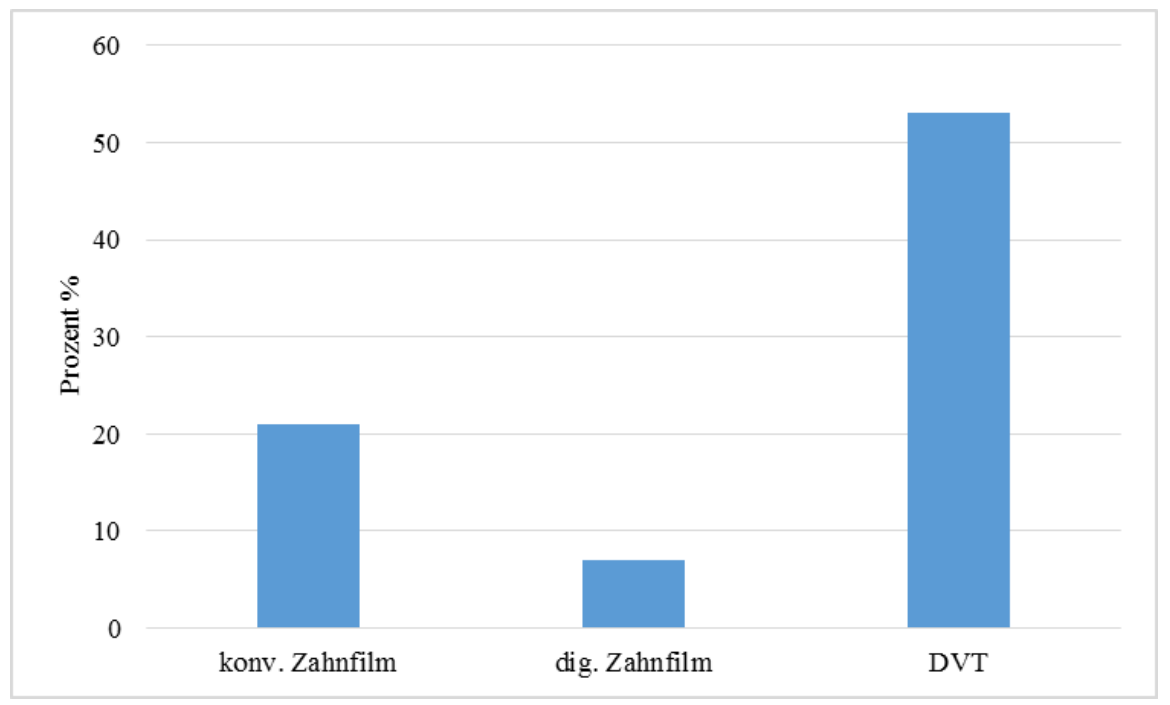

Abbildung 19: Implantatdurchmesser: Darstellung des prozentualen Anteils der korrekt gemessenen Implantatdurchmesser (inkl. Abweichung $+/-0,1 \mathrm{~mm}$ ) in den drei radiologischen Verfahren

\begin{tabular}{|l|c|}
\hline Röntgenologisches Verfahren & $\begin{array}{l}\text { Messabweichung bei der Durchmesserbestimmung der zehn } \\
\text { Implantate (Mittelwerte) }\end{array}$ \\
\hline konventioneller Zahnfilm & $+0,61$ (Standardfehler $\pm 0,14)$ \\
\hline digitaler Zahnfilm & $-0,32$ (Standardfehler $\pm 0,02)$ \\
\hline digitale Volumentomographie (DVT) & $-0,05$ (Standardfehler $\pm 0,03)$ \\
\hline
\end{tabular}

Tabelle 7: Bei der Bestimmung der Implantatdurchmesser wurden in den drei Methoden unterschiedliche Mittelwerte der Messabweichungen ermittelt. Sie werden für jede röntgenologische Methode aufgeführt, in Klammern befindet sich der jeweilige Standardfehler. Eine graphische Darstellung zu dieser Tabelle folgt in Abb.20. 
Boxplot für die Messabweichung der Implantatdurchmesser (Mittelwerte) in mm

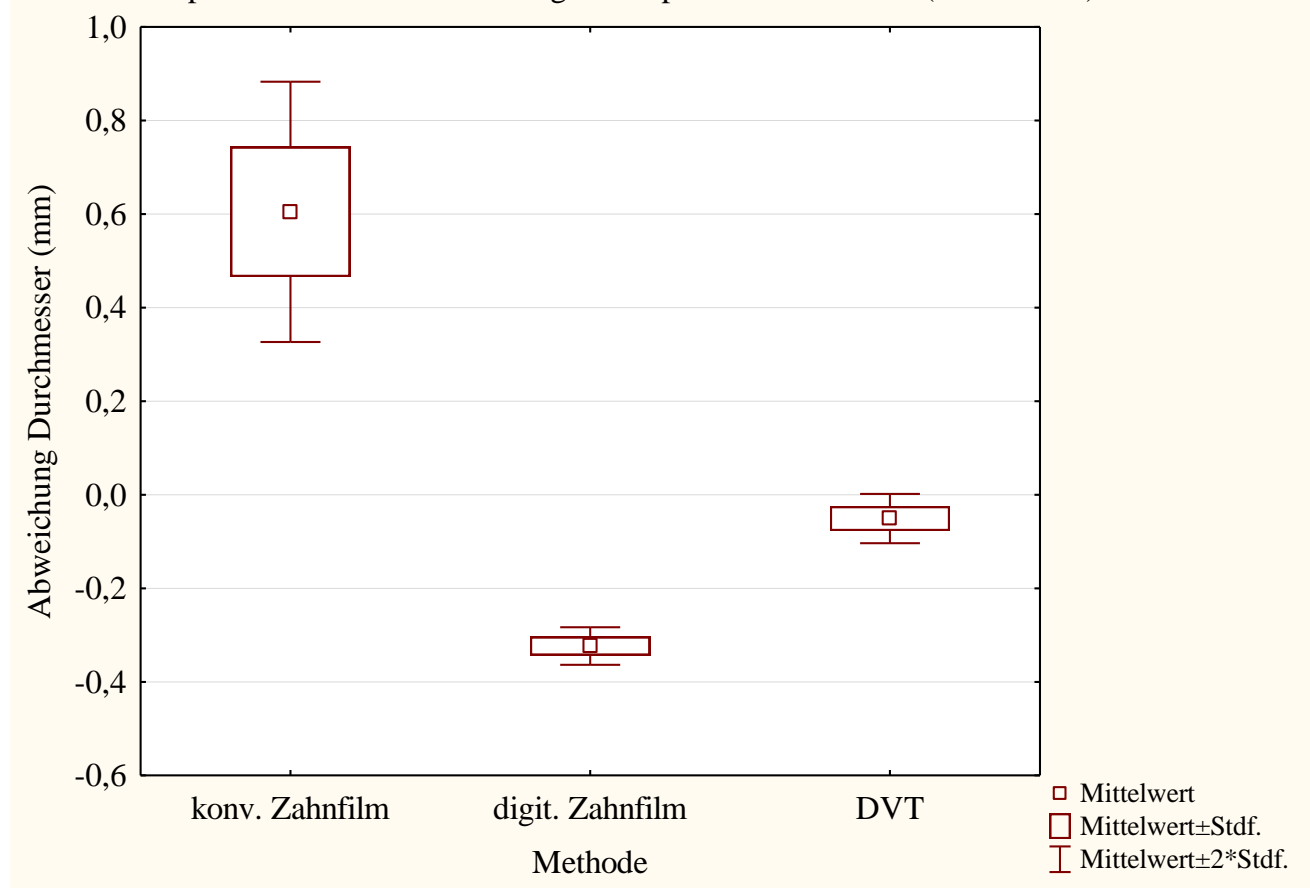

Abbildung 20: Dargestellt sind die Mittelwerte der Messabweichungen der Implantatdurchmesser. Die Mittelwerte und Standardfehler unterscheiden sich in den drei Methoden. In dieser Grafik werden sie einander gegenüber gestellt. Die Null-Linie entspricht der Herstellerangabe der Implantatdurchmesser. Für jede Röntgenmethode zeigen die Mittelwerte, Boxen und Whisker die Verteilung der Messabweichungen von der Herstellerangabe. Die Mittelwerte und Standardfehler sind Tab. 7 zu entnehmen. 


\subsubsection{Evaluation der iatrogen gesetzten Alveolar- und Implantatdefekte}

Es wurde untersucht, ob die verschiedenen Defekte im Alveolarknochen und an den Implantaten mit Hilfe der drei unterschiedlichen Röntgenmethoden von den Untersuchern richtig erkannt und klassifiziert werden konnten.

\subsubsection{Alveolardefekte}

In den drei Methoden unterschieden sich die Auswertungen der korrekt klassifizierten Alveolardefekte nicht signifikant voneinander ( $\mathrm{p}=0,21$; Fisher's exact test).

Zunächst wurde untersucht, wie viele der fünfzig vorhandenen Defekte von den Untersuchern in den zu vergleichenden Methoden korrekt erkannt wurden. In den konventionellen Zahnfilmaufnahmen wurden $64 \%$ der Defekte richtig erkannt. In den digitalen Zahnfilmaufnahmen wurden $66 \%$ der vorhandenen Defekte erkannt. In der digitalen Volumentomographie gelang es den Untersuchern $50 \%$ der vorhandenen Defekte zu beobachten. In den zweidimensionalen Methoden konnten in den konventionellen Zahnfilmaufnahmen $47 \%$ der richtig erkannten Alveolardefekte korrekt klassifiziert werden. In den digitalen Zahnfilmaufnahmen diagnostizierten die Untersucher $40 \%$ der erkannten Defekte korrekt. Durch die dreidimensionale Röntgenmethode DVT wurden 68 \% der richtig erkannten Defekte der korrekten Defektklasse zugeordnet (Abb. 21). 


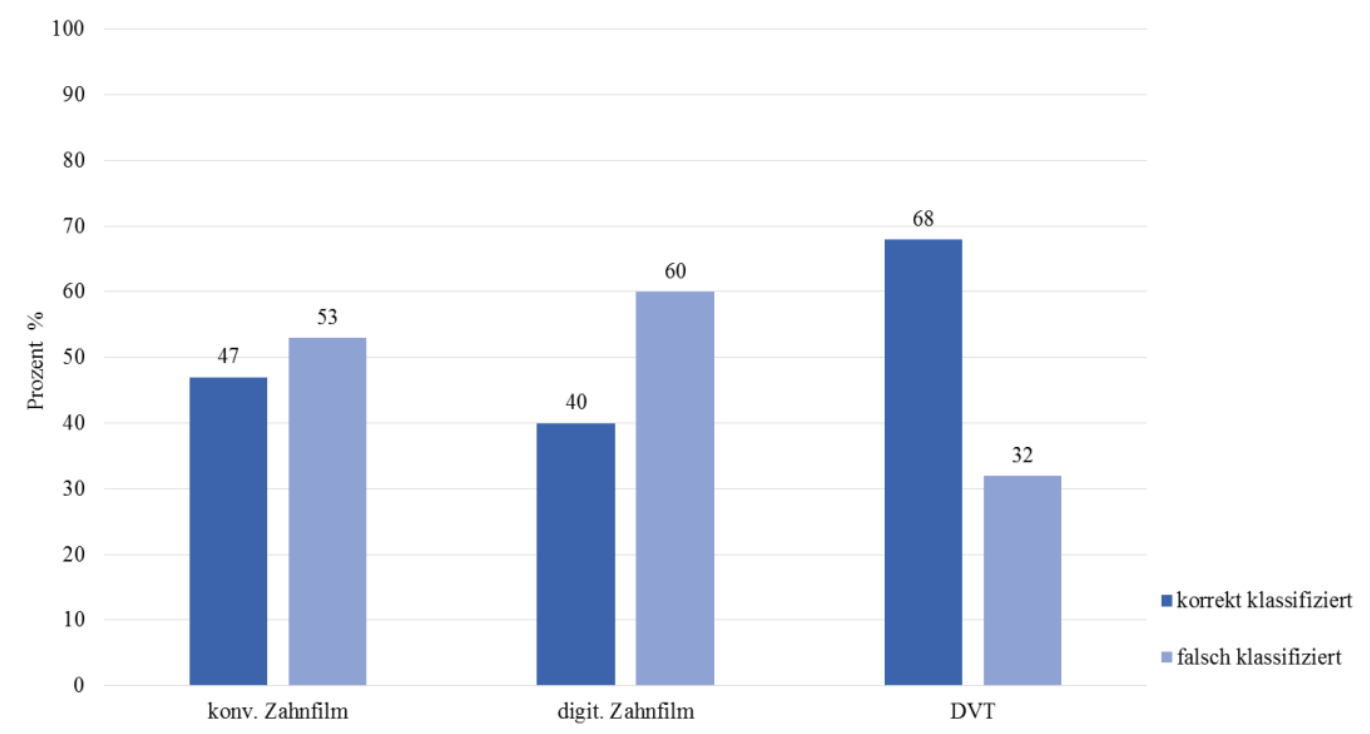

Abbildung 21: Dargestellt werden die von den Untersuchern erkannten Alveolardefekte, unterteilt in korrekt und falsch klassifizierte Defekte. Dabei werden die Beurteilungen der 10 Untersucher für jede Röntgenmethode gesondert dargestellt. Die unterschiedlichen Defektmorphologien der Knochendefekte werden zusammengefasst abgebildet.

Der Alveolardefekt A (1 mm tiefe keilförmige Bohrung in $4 \mathrm{~mm}$ Höhe) war in Präparat $2 \mathrm{zu}$ erkennen. In diesem Präparat wurde der Defekt nur in den Zahnfilmaufnahmen erkannt und korrekt klassifiziert.
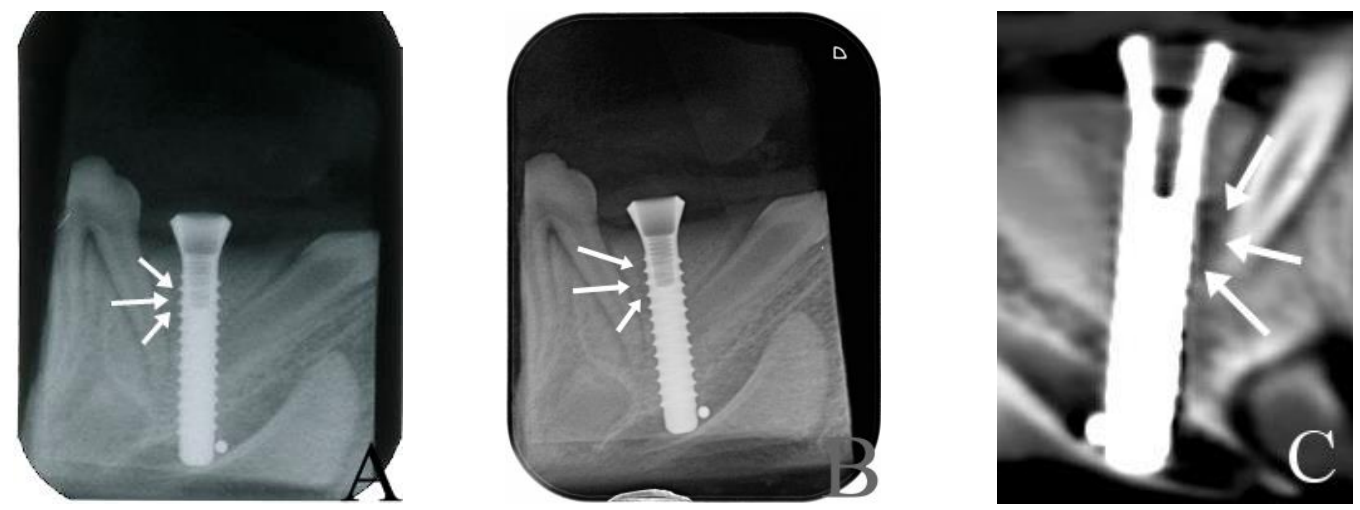

Abbildung 22: Alveolardefekt A in Präparat 2 (keilförmige Bohrung, $1 \mathrm{~mm}$ tief in $4 \mathrm{~mm}$ Höhe): Die Pfeile zeigen auf die knöcherne Begrenzung der Alveolardefektes. A: konv. Zahnfilm B: dig. Zahnfilm C: Ausschnitt aus DVT (koronaler Schnitt) 


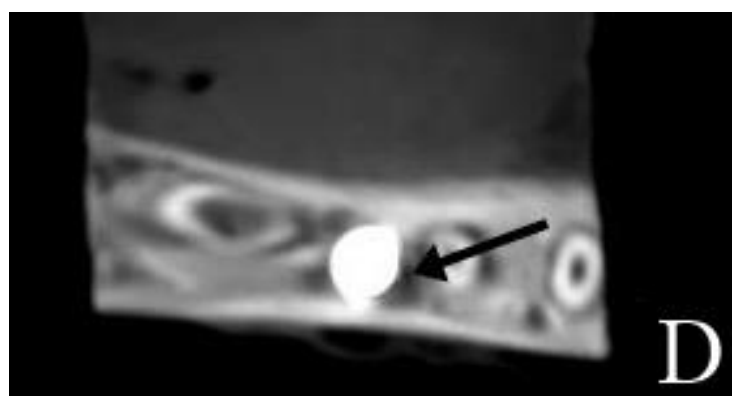

D: Ausschnitt aus DVT (axialer Schnitt): Der Pfeil zeigt auf den keilförmigen Alveolardefekt in direkter Nachbarschaft zur Implantatoberfläche

Im Gegensatz dazu wurde der Alveolardefekt B (Längsrille von $1 \mathrm{~mm}$ Tiefe und $\leq 10 \mathrm{~mm}$ Länge) in Präparat 12 in den DVT-Aufnahmen häufiger korrekt diagnostiziert.
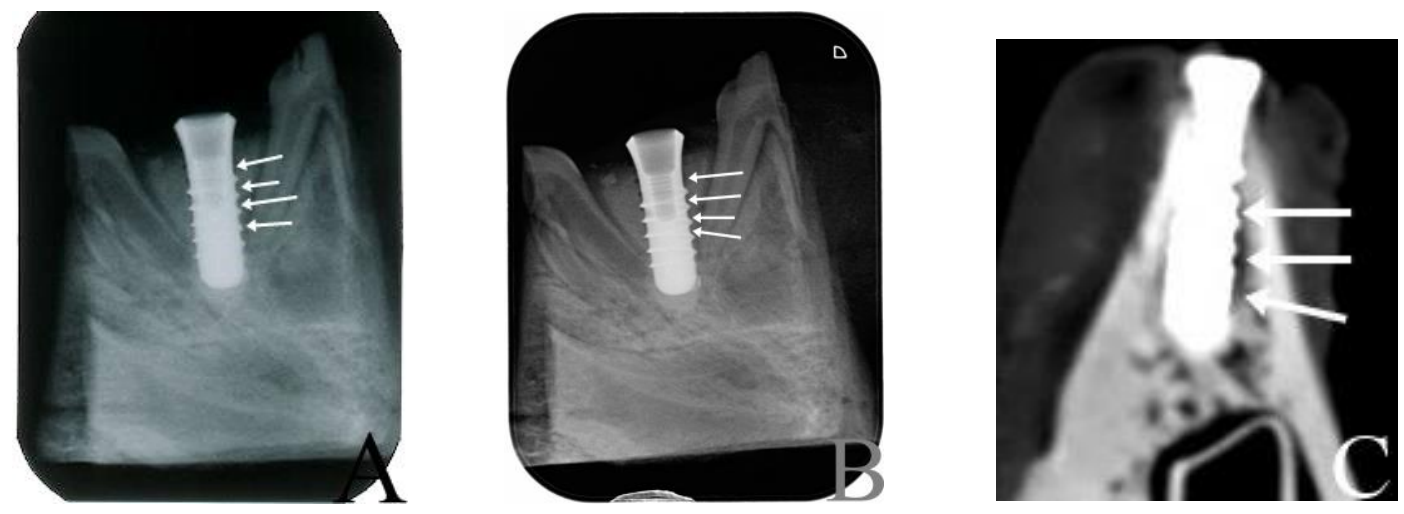

Abbildung 23: Alveolardefekt B in Präparat 12 (Längsrille, $1 \mathrm{~mm}$ Tiefe und $\leq 10 \mathrm{~mm}$ Länge): Die Pfeile zeigen auf die knöcherne Begrenzung des Defektes A: konv. ZF B: dig. ZF C: Ausschnitt aus DVT (sagittaler Schnitt) 
Der Defekt „Bonesplitting“ (Präparat 10) konnte in den zweidimensionalen Verfahren häufiger korrekt klassifiziert werden als in den dreidimensionalen DVT-Aufnahmen (Abb. 24).
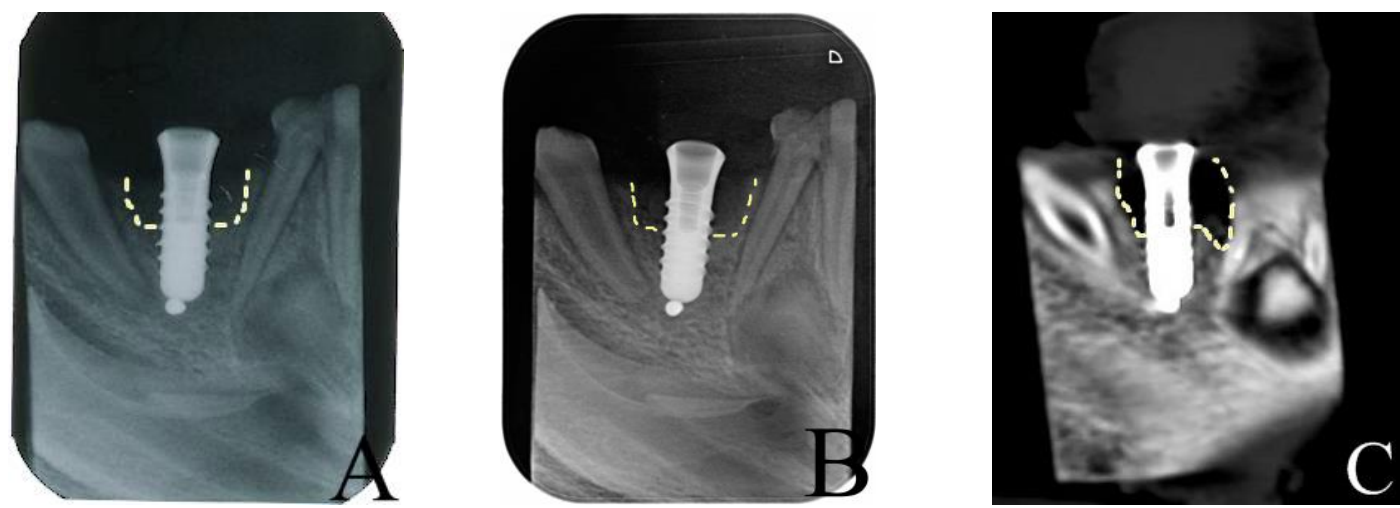

Abbildung 24:Alveolardefekt „Bonesplitting“ im Präparat 10 (siehe gestrichelte Markierung) A: konv. ZF B: dig. ZF C: Ausschnitt aus DVT (koronaler Schnitt)

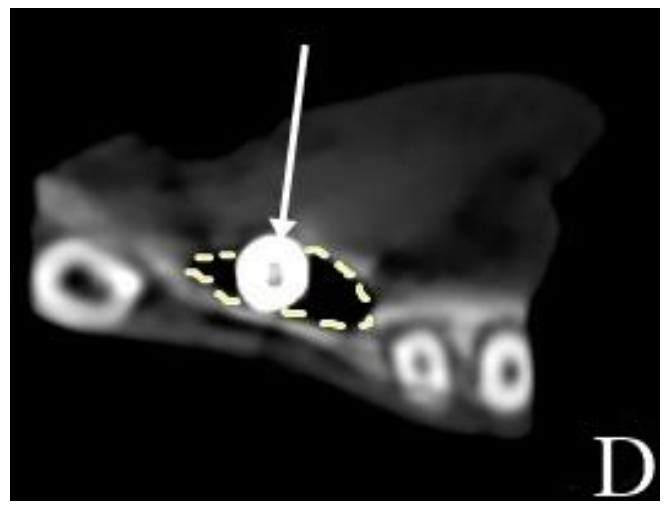

D: Ausschnitt aus DVT (axialer Schnitt): Der Pfeil zeigt auf den Implantatkopf, die gestrichelte Linie markiert den Bereich des „Bonesplitting“

In Präparat 13 liegt der Alveolardefekt im Sinne einer Überdimension vor. Es wurde eine Kavitätenbohrung für eine Implantatlänge von $12 \mathrm{~mm}$ durchgeführt, jedoch ein Implantat der Länge $8 \mathrm{~mm}$ inseriert. Dieser Defekt wurde in allen Verfahren häufig erkannt. In den konventionellen Zahnfilmaufnahmen lag die Erkennungsquote bei $100 \%$, in den beiden anderen Methoden wurde der Alveolardefekt in $80 \%$ der Fälle korrekt diagnostiziert (Abb. 25). 

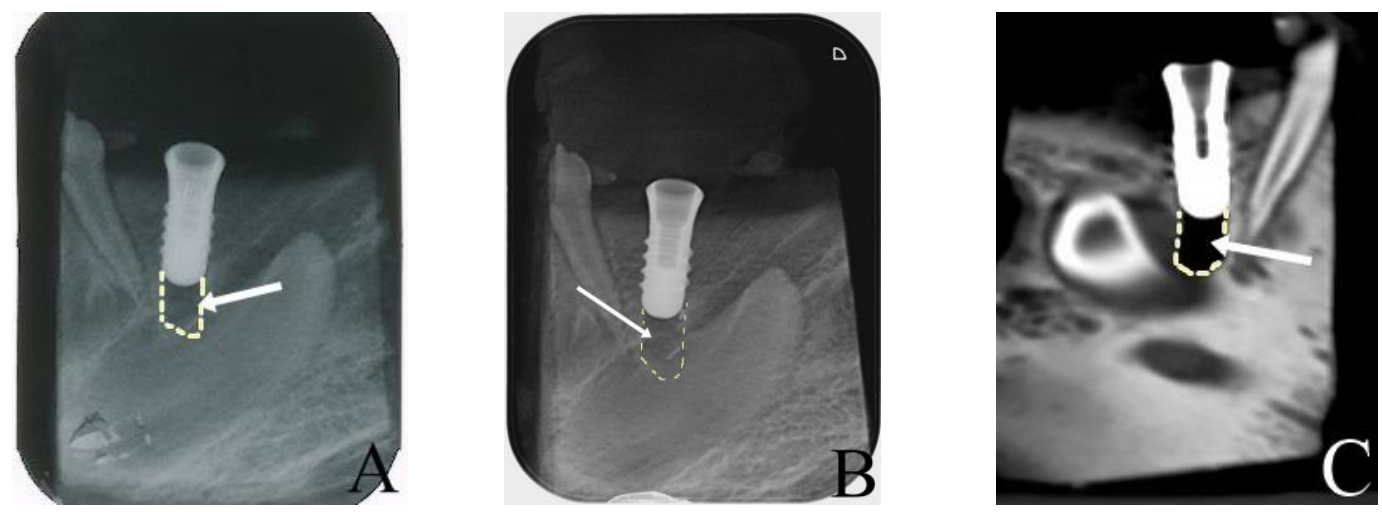

Abbildung 25: Alveolardefekt „Überdimension“ im Präparat 13 (siehe Pfeile u. gestrichelte Markierung) A: konv. ZF B: dig. ZF C: Ausschnitt aus DVT (koronaler Schnitt)

Der Defekt „Ersatz, lingual perforiert“ (Präparat 14) mit einer Perforation bei Implantatinsertion und anschließender Ersatz durch eine kleineres Implantat konnte nur in den dreidimensionalen DVT-Aufnahmen korrekt diagnostiziert werden. In den zweidimensionalen Verfahren wurde dieser Defekt häufig falsch als Überdimension oder Längsrille (Defekt B) klassifiziert (Abb. 26).
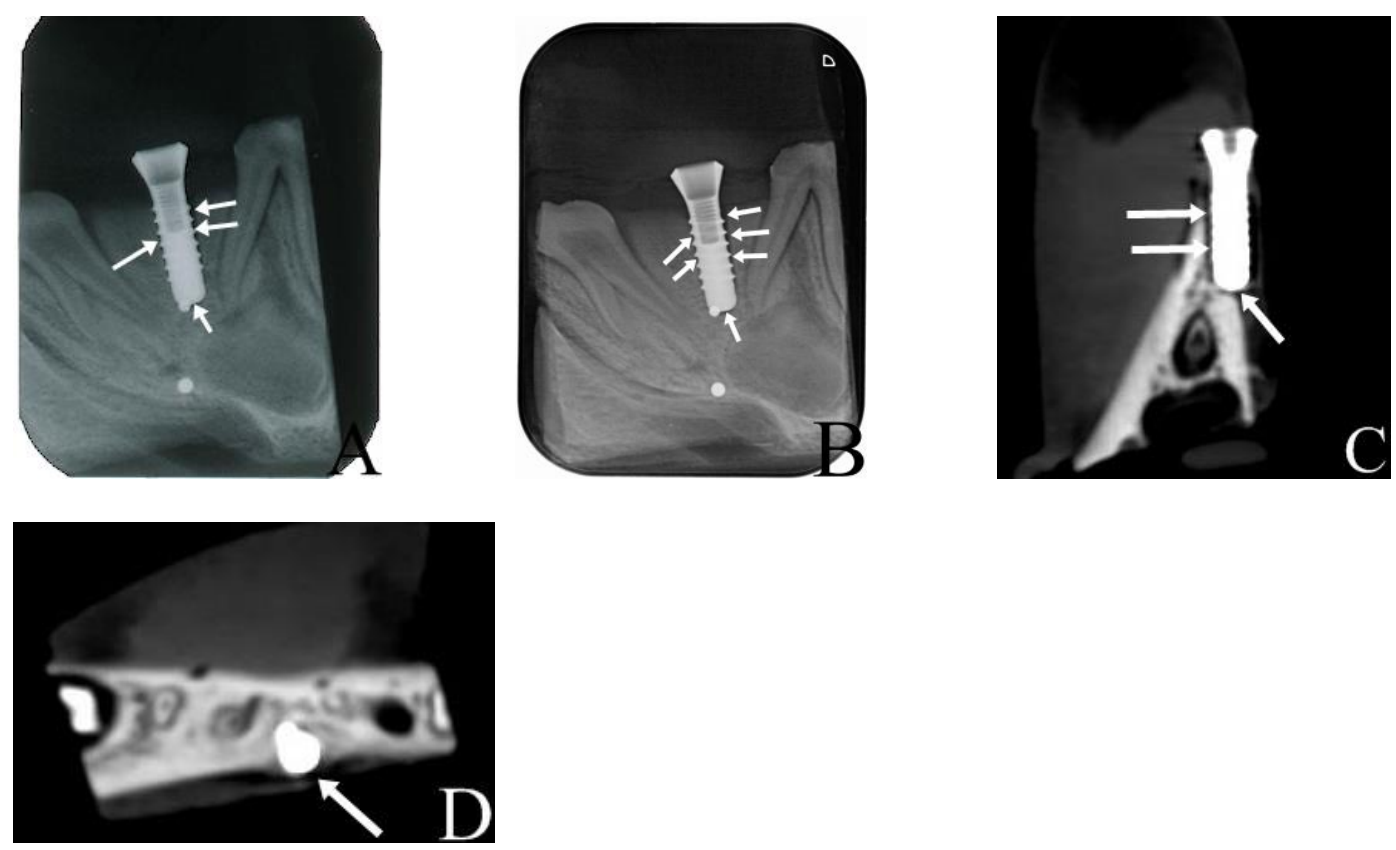

Abbildung 26: Alveolardefekt „Ersatz, lingual perforiert“ in Präparat 14: Die Pfeile zeigen auf die Begrenzungen des Implantatbettes und im apikalen Bereich auf die Perforation A: konv. ZF B: dig ZF C: Ausschnitt aus DVT (sagittaler Schnitt) D: Ausschnitt aus DVT (axialer Schnitt, Pfeil zeigt auf den perforierten Bereich der lingualen Knochenlamelle im apikalen Teil des Implantates) 


\subsubsection{Implantatdefekte}

Die iatrogen produzierten Implantatdefekte konnten mit allen drei Röntgenmethoden dargestellt werden. Die drei Methoden unterschieden sich hinsichtlich der korrekten Erkennung eines Implantatdefektes signifikant voneinander ( $p=0,0002$, Chi-Quadrat-Test). Die zweidimensionalen Methoden ähnelten sich dabei in der Häufigkeit der richtigen erkannten Implantatdefekte. In den konventionellen Zahnfilmaufnahmen erkannten die Untersucher $42 \%$ der vorhandenen Defekte und in den digitalen Zahnfilmaufnahmen handelte es sich um $46 \%$ richtig erkannte Defekte. In der DVT erkannten die Untersucher $10 \%$ der vorhandenen Defekte. Die Evaluationen der Implantatdefekte unterschieden sich in Bezug auf die korrekte Klassifizierung der Implantatdefekte im intermethodischen Vergleich nicht signifikant voneinander ( $p=0,29$; Fisher's exact test). In den zweidimensionalen Verfahren wurden in den konventionellen $67 \%$ und in den digitalen Zahnfilmaufnahmen $65 \%$ der richtig erkannten Implantatdefekte auch korrekt klassifiziert. In den dreidimensionalen DVTAufnahmen konnten $60 \%$ der erkannten Defekte richtig diagnostiziert werden (Abb. 27).

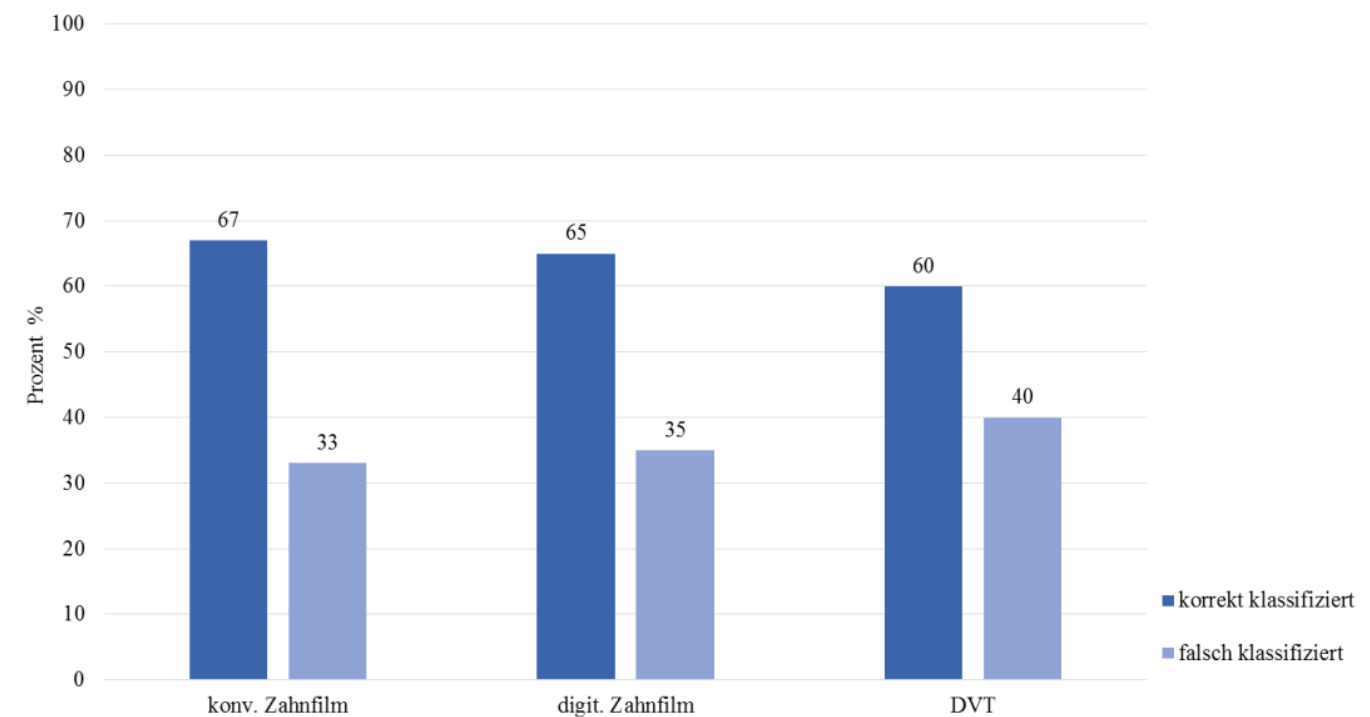

Abbildung 27: Dargestellt werden die von den Untersuchern erkannten Implantatdefekte, unterteilt in korrekt und falsch klassifizierte Defekte. Dabei werden die Beurteilungen der 10 Untersucher für jede Röntgenmethode gesondert dargestellt. Die unterschiedlichen Defektmorphologien der Knochendefekte werden zusammengefasst abgebildet. 
Implantatdefekt A (Längsrille in Windungstiefe) in den Präparaten 5 und 14 konnte durch die Untersucher nur in den zweidimensionalen Röntgenmethoden korrekt diagnostiziert werden (Abb. 28). In den konventionellen Zahnfilmaufnahmen konnte der Defekt in $5 \%$ korrekt klassifiziert werden, in den digitalen Zahnfilmaufnahmen diagnostizierten die Untersucher in $10 \%$ die korrekte Defektklasse. In den DVT-Aufnahmen wurde in $90 \%$ der Beobachtungen kein Defekt erkannt und in jeweils $5 \%$ wurde der Defekt falsch als Querrille oder entfernte Windung klassifiziert. Zudem wurde die Längsrille in Windungstiefe nur im Präparat 5 erkannt.
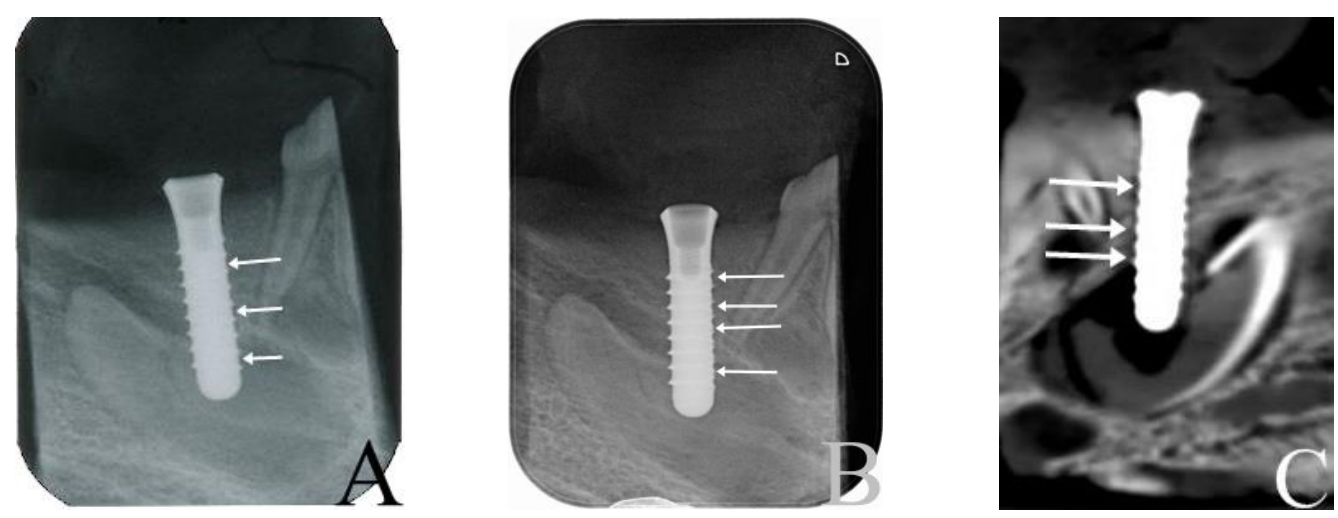

Abbildung 28: Implantatdefekt A (Längsrille in Windungstiefe) in Präparat 5 (siehe Pfeile) A: konv. ZF B: dig. ZF C:Ausschnitt aus DVT (koronaler Schnitt)

Der Implantatdefekt B (Planierungen der Implantatoberfläche) in Präparat 3 wurde ebenfalls nur in den zweidimensionalen Röntgenmethoden von den Untersuchern korrekt klassifiziert (Abb. 29). In den konventionellen Zahnfilmaufnahmen klassifizierten die Untersucher $50 \%$ der erkannten Defekte korrekt. In den digitalen Zahnfilmaufnahmen wurden $30 \%$ korrekt klassifiziert. In den dreidimensionalen Aufnahmen wurde in diesem Präparat kein Implantatdefekt erkannt. 

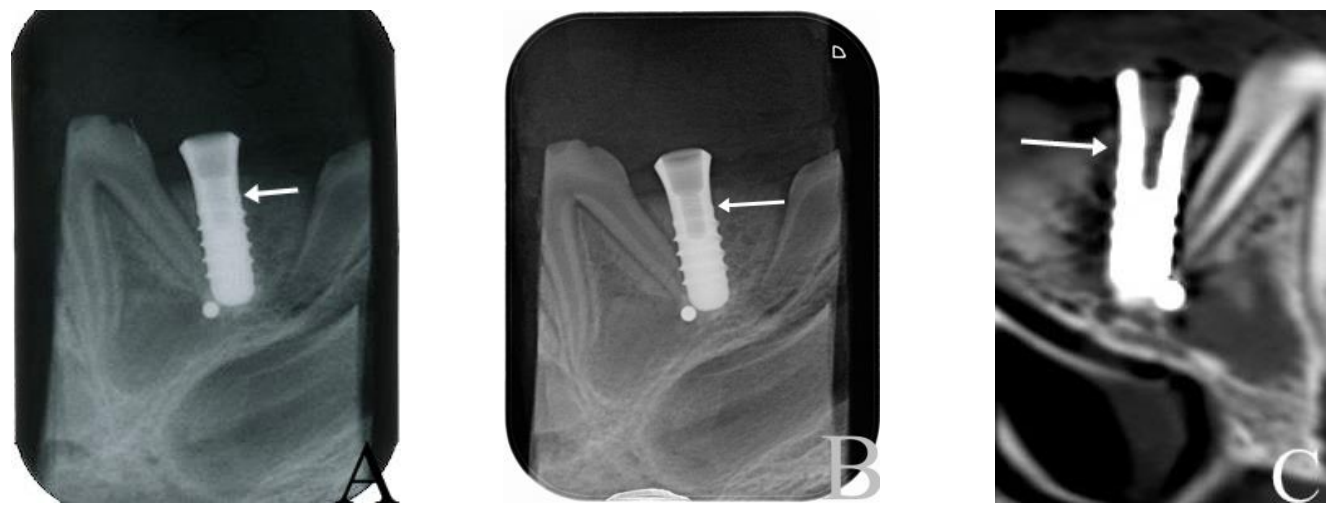

Abbildung 29: Implantatdefekt B (obere Windung planiert) in Präparat 3 (Pfeil zeigt auf den Defekt) A: konv. ZF B: dig. ZF C:Ausschnitt aus DVT (koronaler Schnitt) 
Die Querrille als Implantatdefekt C in Präparat 8 und 12 wurde in $45 \%$ der konventionellen Zahnfilmaufnahmen korrekt diagnostiziert. In den digitalen Zahnfilmaufnahmen klassifizierten die Untersucher 50 \% der Defekte korrekt und in den DVT-Aufnahmen konnten $15 \%$ der erkannten Defekte korrekt zugeordnet werden. Die Querrille wurde dabei häufig nur im Präparat 8 korrekt klassifiziert und in Präparat 12 als planierte Windung fehldiagnostiziert.
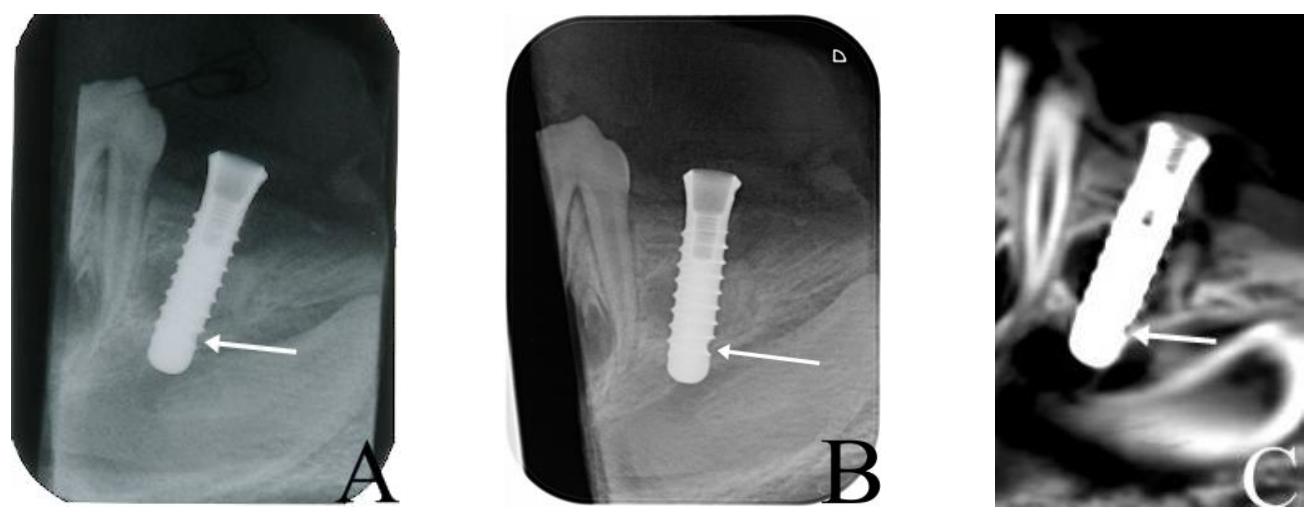

Abbildung 30: Implantatdefekt C (Querrille, zwischen den unteren Windungen) in Präparat 8 (Pfeil zeigt auf den Defekt) A: konv. ZF B: dig. ZF C:Ausschnitt aus DVT (koronaler Schnitt)
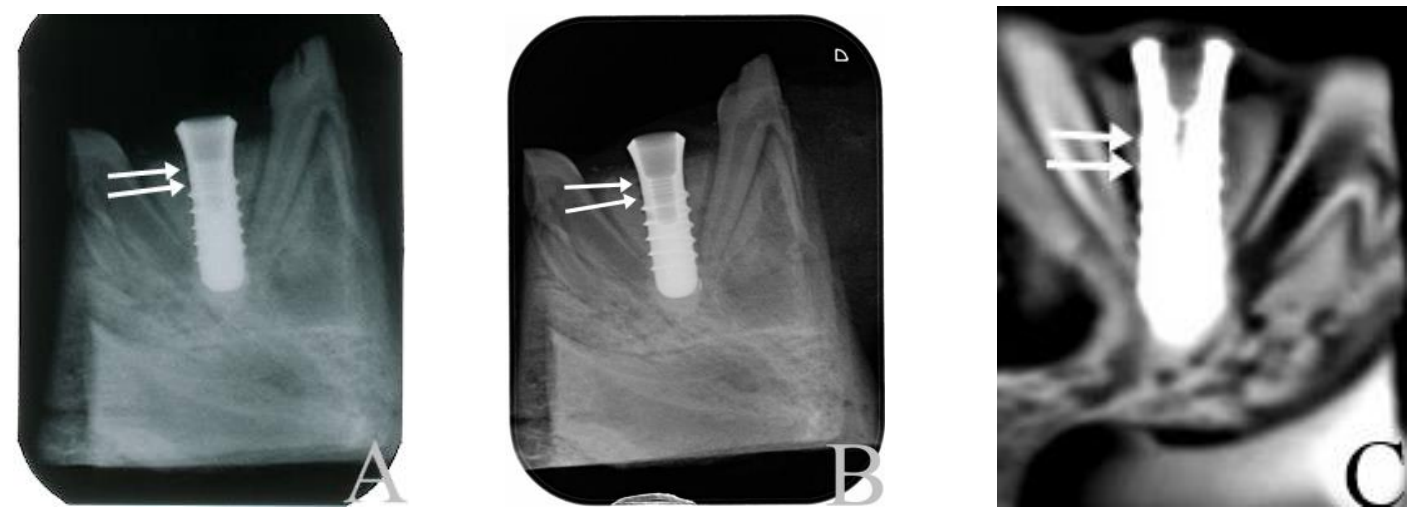

Abbildung 31: Implantatdefekt C (Querrille, zwischen den oberen Windungen) in Präparat 12 (Pfeil zeigt auf den Defekt) A: konv. ZF B: dig. ZF C:Ausschnitt aus DVT (koronaler Schnitt) 


\subsubsection{Fremdkörper}

Die verschiedenen Fremdmaterialien in den Präparaten unterschieden sich stark in ihrer Dichte. Daraus resultiert auch eine unterschiedlich starke Röntgenopazität dieser Körper in den Röntgenaufnahmen der drei bildgebenden Verfahren. Mit jeder der drei verwendeten Methoden war eine Darstellung der unterschiedlichen Materialien möglich. In $92 \%$ aller Befunde wurde ein Vorhandensein von Fremdmaterialien richtig erkannt. Dabei erkannten die Untersucher in den konventionellen Zahnfilmaufnahmen 77 von 80 vorhandenen Fremdkörpern richtig und in den digitalen Zahnfilmaufnahmen erkannten sie 79 von 80. In den DVT-Aufnahmen wurden 65 der 80 vorhandenen Fremdkörper erkannt. In den konventionellen Zahnfilmaufnahmen wurden $73 \%$ und in den digitalen Zahnfilmaufnahmen $74 \%$ der richtig erkannten Fremdmaterialien korrekt klassifiziert, in der digitalen Volumentomographie (DVT) waren es $77 \%$ der richtig erkannten Fremdmaterialien. Das korrekte Auffinden von Fremdmaterialien in den Präparaten unterschied sich im intermethodischen Vergleich nicht signifikant voneinander $(\mathrm{p}=0,08$; Chi-Quadrat-Test) (Abb. 32).

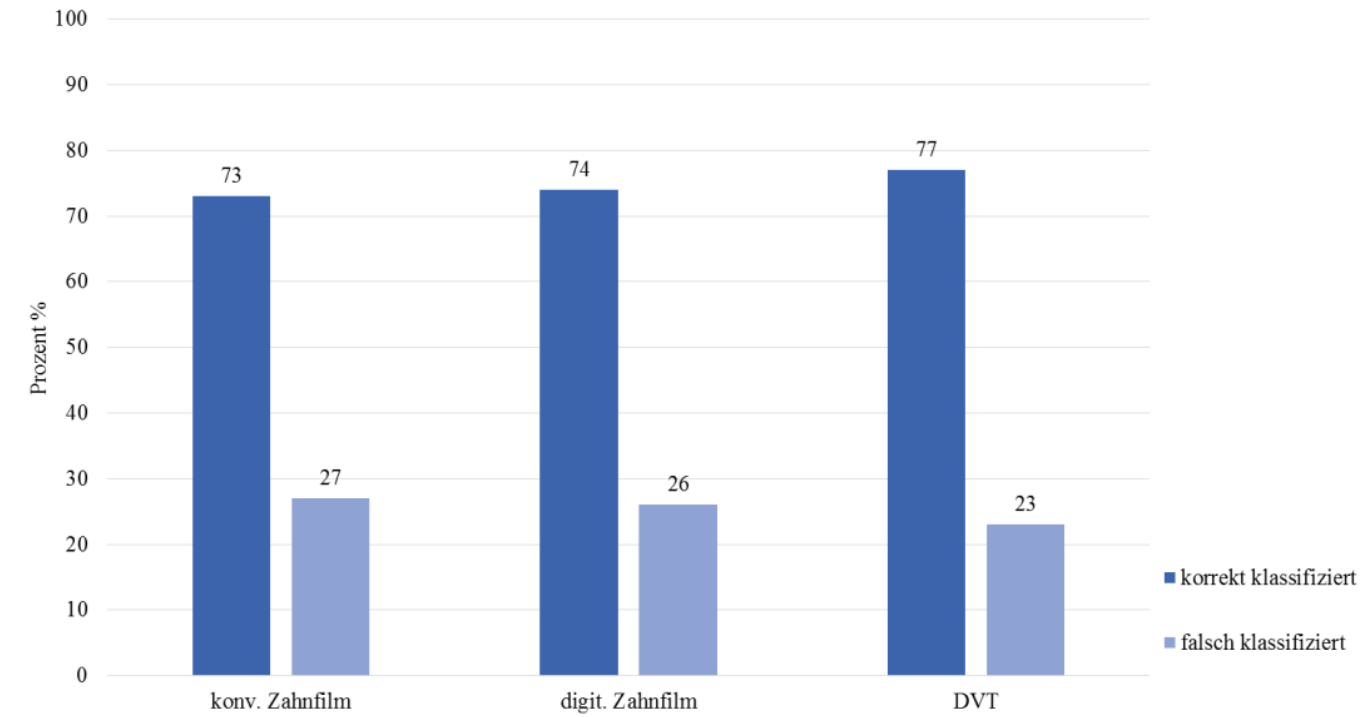

Abbildung 32: Dargestellt werden die von den Untersuchern erkannten Fremdkörper, unterteilt in korrekt klassifizierte und falsch klassifizierte Fremdkörper. Die Auswertungen der Untersucher sind für jede der drei untersuchten Methoden gesondert abgebildet. Die unterschiedlichen Arten der Fremdkörper wurden zusammengefasst dargestellt. 
Die verschiedenen Fremdkörper wurden unterschiedlich häufig erkannt und korrekt klassifiziert. Dabei wurde deutlich, dass die Stahlkugel als Fremdkörper in allen Methoden sehr oft erkannt und ebenso korrekt klassifiziert wurde. Im Vergleich der drei bildgebenden Verfahren ergab sich bezüglich der korrekten Klassifizierung der Stahlkugel als Fremdkörper kein signifikanter Unterschied ( $p=0,865$; Chi-Quadrat-Test).

Die Fremdkörper Guttapercha und B-TCP wurden im Gegensatz zu der Stahlkugel weitaus seltener erkannt und klassifiziert. Im intermethodischen Vergleich bezüglich der korrekten Erkennung der Guttaperchastifte ergab sich ein signifikanter Unterschied $(\mathrm{p}=0,025$; ChiQuadrat-Test). Die korrekte Klassifizierung von B-TCP unterschied sich im Vergleich der drei Röntgenverfahren nicht signifikant ( $\mathrm{p}=0,412$; Chi-Quadrat-Test) (Abb. 33).

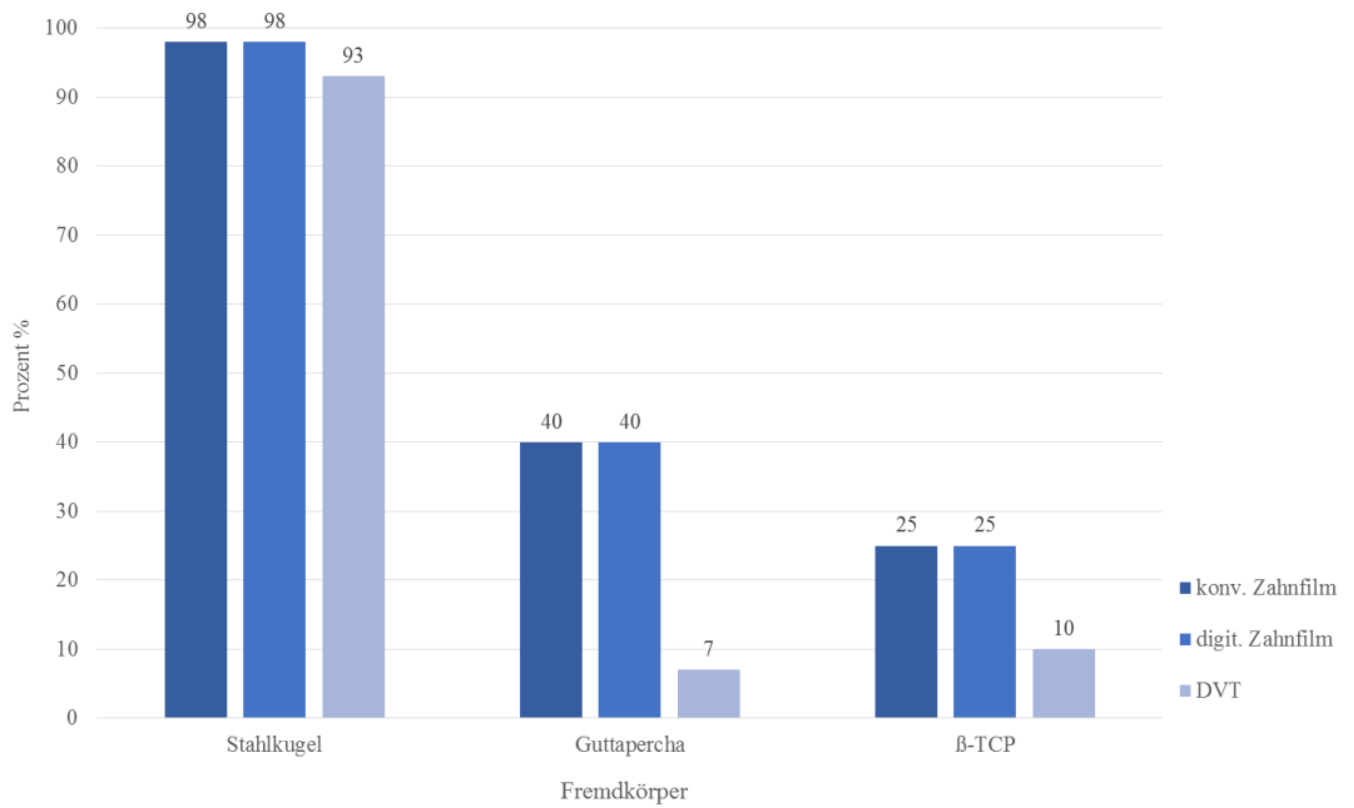

Abbildung 33: Die Abbildung zeigt die Häufigkeit der korrekten Fremdkörperklassifizierung in den drei Röntgenmethoden. Die drei Arten von Fremdkörpern sind dabei gesondert aufgeführt. Die drei Balken stellen den Anteil der korrekten Befunde dar, die in der jeweiligen Methode für den Fremdkörper ermittelt wurden. 


\section{Stahlkugel}

Die Stahlkugeln wurden in zwei Durchmessern $(1 \mathrm{~mm} ; 1,6 \mathrm{~mm})$ in unterschiedlichen Verteilungen in die Schweinekieferpräparate eingebracht. Insgesamt konnten acht Kugeln erkannt werden. Bei fünf Stahlkugeln betrug der Durchmesser $1 \mathrm{~mm}$ (Präparat 2, 3 und 4). Die verbleibenden drei Kugeln hatten einen Durchmesser von 1,6 mm (Präparat 11). In den Präparaten 10 und 14 war jeweils eine Kugel von jedem Durchmesser vorhanden. Die korrekte Klassifizierung der Stahlkugeln als Fremdkörper ergab keinen signifikanten Unterschied ( $\mathrm{p}=0,865 ;$ Chi-Quadrat-Test). Die Stahlkugeln wurden in den konventionellen Zahnfilmaufnahmen in $74 \%$ der Befunde korrekt klassifiziert. In den digitalen Zahnfilmaufnahmen bewerteten die Untersucher $73 \%$ korrekt als Stahlkugel. In den dreidimensionalen DVT-Aufnahmen wurden $70 \%$ der vorhandenen Kugeln korrekt klassifiziert.
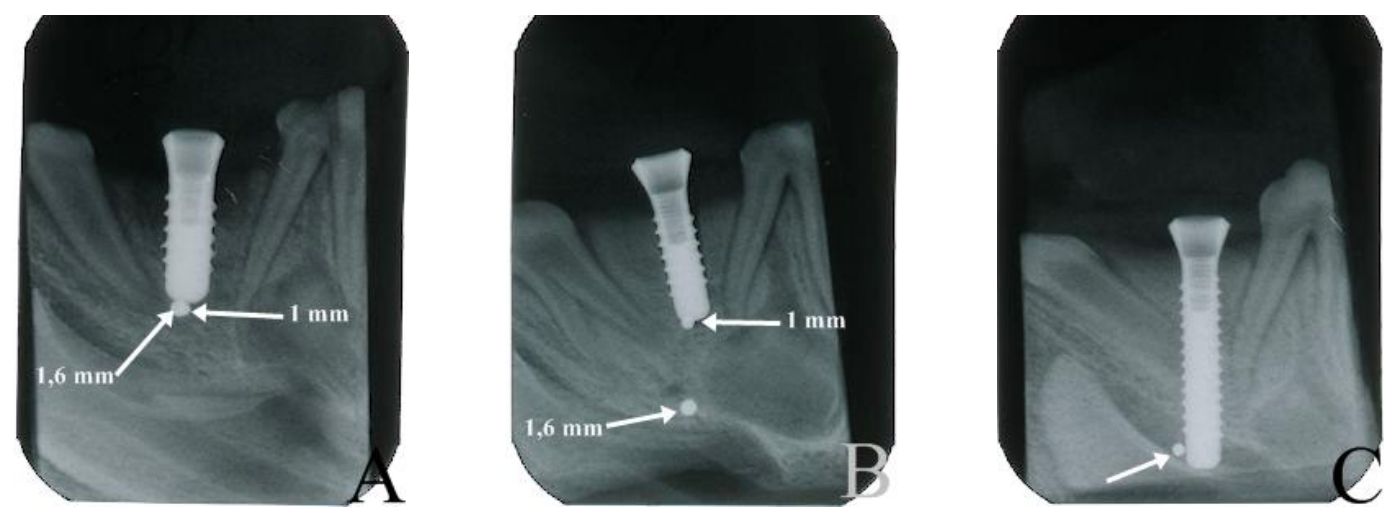

Abbildung 34: konventionelle Zahnfilmaufnahmen; Fremdmaterial Stahlkugel (siehe Pfeil) A: Präparat 10, Kugel $\varnothing 1,6 \mathrm{~mm}$ und $\varnothing 1 \mathrm{~mm}$ B: Präparat $14, \varnothing 1,6 \mathrm{~mm}$ und $\varnothing 1 \mathrm{~mm}$ C: Präparat 4, $\varnothing 1 \mathrm{~mm}$
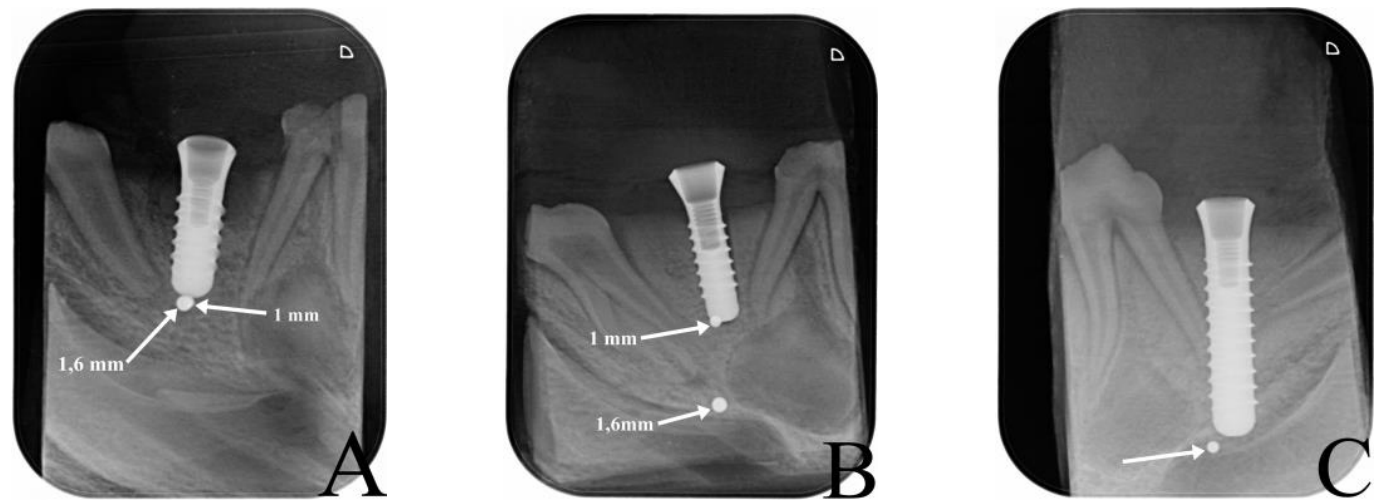

Abbildung 35: digitale Zahnaufnahmen; Fremdmaterial Stahlkugel (siehe Pfeil) A: Präparat 10, Kugel $\varnothing ~ 1,6 \mathrm{~mm}$ und $\varnothing 1 \mathrm{~mm}$ B: Präparat $14, \varnothing 1,6 \mathrm{~mm}$ und $\varnothing 1 \mathrm{~mm} \mathrm{C}$ : Präparat 4, $\varnothing 1 \mathrm{~mm}$ 

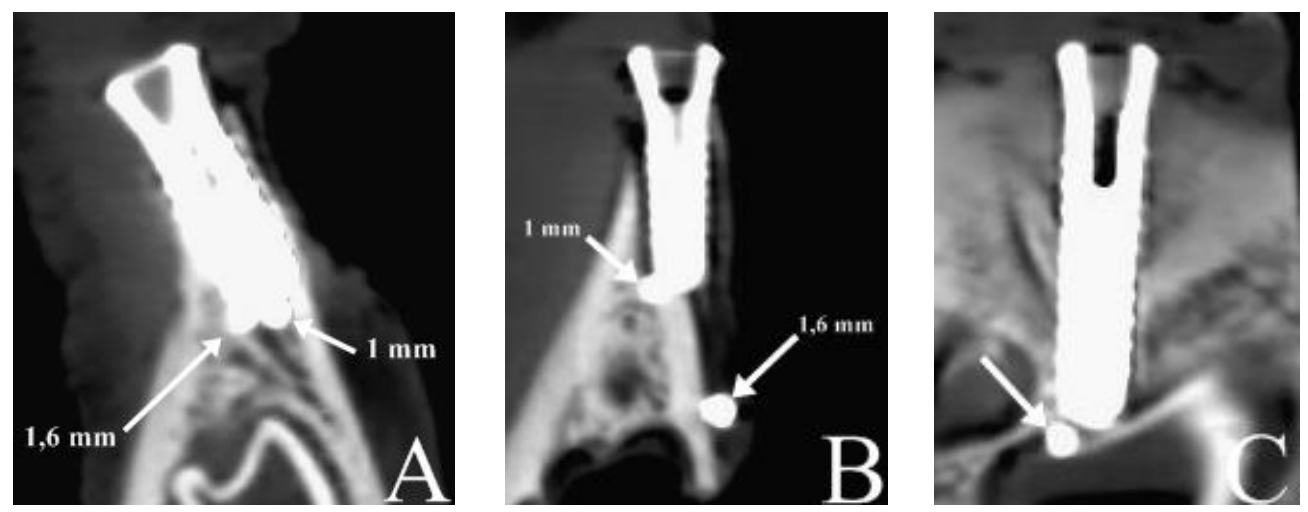

Abbildung 36: Ausschnitte aus DVT-Aufnahmen; Fremdmaterial Stahlkugel (siehe Pfeil) A: Präparat 10, Kugel $\varnothing 1,6 \mathrm{~mm}$ und $\varnothing 1 \mathrm{~mm}$ (sagittaler Schnitt) B: Präparat $14, \varnothing 1,6 \mathrm{~mm}$ und $\varnothing 1 \mathrm{~mm}$ (sagittaler Schnitt)

C: Präparat 4, $\varnothing 1 \mathrm{~mm}$ (koronaler Schnitt)

Die Präzision der Durchmesserbestimmung der Stahlkugeln ergab im intermethodischen Vergleich einen signifikanten Unterschied $(\mathrm{p}<0,001)$. Dabei ergab sich auch bei der Genauigkeit der Messergebnisse ein signifikanter Unterschied für die beiden unterschiedlichen Kugeldurchmesser $1 \mathrm{~mm}$ (Tab. 8; Abb. 37) und 1,6 mm (p = 0,014) (Tab. 9; Abb. 38).

\begin{tabular}{|l|c|}
\hline Röntgenologisches Verfahren & $\begin{array}{l}\text { Messabweichung bei der Durchmesserbestimmung der } \\
\text { Stahlkugeln } \varnothing 1 \mathrm{~mm} \text { in mm (Mittelwerte) }\end{array}$ \\
\hline konventioneller Zahnfilm & $+0,01$ (Standardfehler $\pm 0,07)$ \\
\hline digitaler Zahnfilm & $-0,02$ (Standardfehler $\pm 0,05)$ \\
\hline digitale Volumentomographie (DVT) & $+0,39$ (Standardfehler $\pm 0,07)$ \\
\hline
\end{tabular}

Tabelle 8: Dargestellt wird die Messabweichung für den Stahlkugeldurchmesser Ø $1 \mathrm{~mm}$. Es sind die Mittelwerte der Messabweichung und der zugehörige Standardfehler für jedes röntgenologische Verfahren aufgeführt. Die graphische Darstellung dieser Tabelle findet sich in Abb. 37. 
Boxplot für die Messabweichung der Stahlkugel $\varnothing 1 \mathrm{~mm}$ (Mittelwerte in mm)

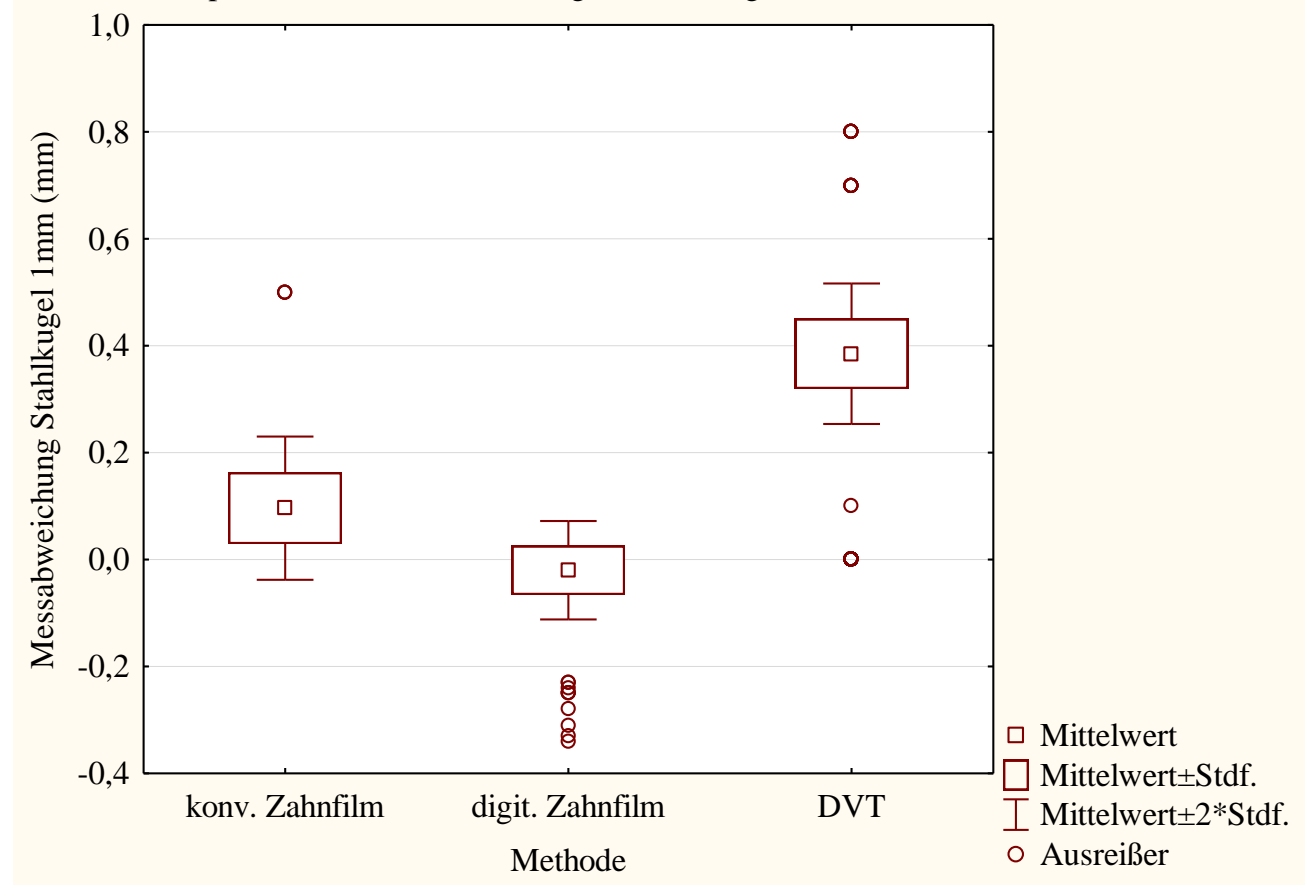

Abbildung 37: Messabweichung der Stahlkugeln $\varnothing 1 \mathrm{~mm}$. Die Null-Linie stellt den reellen Durchmesser laut Herstellerangabe dar. Der Mittelwert, die Box und die Whisker bilden gesondert für jede getestete Methode die Messabweichung des Stahlkugeldurchmessers ab.

Der Tabelle Tab. 8 und dem Boxplot in Abb. 37 sind zu entnehmen, dass der Mittelwert und der Standardfehler in den DVT-Aufnahmen stärker von der Herstellerangabe $1 \mathrm{~mm}$ divergiert als in den zweidimensionalen Aufnahmen. Der Großteil der Messungen wurde in fast allen Methoden bei der $1 \mathrm{~mm}$-Stahlkugel länger gemessen als die Herstellerangabe angab.

Bei der Messung mit der Stahlkugel von 1,6 mm Durchmesser lag keine auffällige Abweichung der DVT-Aufnahmen gegenüber der anderen Methoden vor. In Tab. 9 und Abb. 38 ist dargestellt, dass in den DVT-Aufnahmen und in den konventionellen Zahnfilmaufnahmen eine weniger starke Messabweichung vorlag als in den digitalen Zahnfilmaufnahmen. Insgesamt wurden die Stahlkugeln mit dem Durchmesser 1,6 mm in allen Methoden kürzer gemessen als die Angabe des Herstellers. 


\begin{tabular}{|l|c|}
\hline Röntgenologisches Verfahren & $\begin{array}{c}\text { Messabweichung bei der Durchmesserbestimmung der } \\
\text { Stahlkugeln } \varnothing 1,6 \mathrm{~mm} \text { (Mittelwerte) }\end{array}$ \\
\hline konventioneller Zahnfilm & $-0,07$ (Standardfehler $\pm 0,03)$ \\
\hline digitaler Zahnfilm & $-0,15$ (Standardfehler $\pm 0,3)$ \\
\hline digitale Volumentomographie (DVT) & $-0,04$ (Standardfehler $\pm 0,05)$ \\
\hline
\end{tabular}

Tabelle 9: Dargestellt wird die Messabweichung für die Stahlkugeldurchmesser Ø 1,6 mm. Es sind die Mittelwerte der Messabweichung und der zugehörige Standardfehler für jedes röntgenologische Verfahren aufgeführt. Die graphische Darstellung dieser Tabelle findet sich in Abb. 38.

Boxplot für die Messabweichung der Stahlkugel Ø 1,6 mm (Mittelwerte in mm)

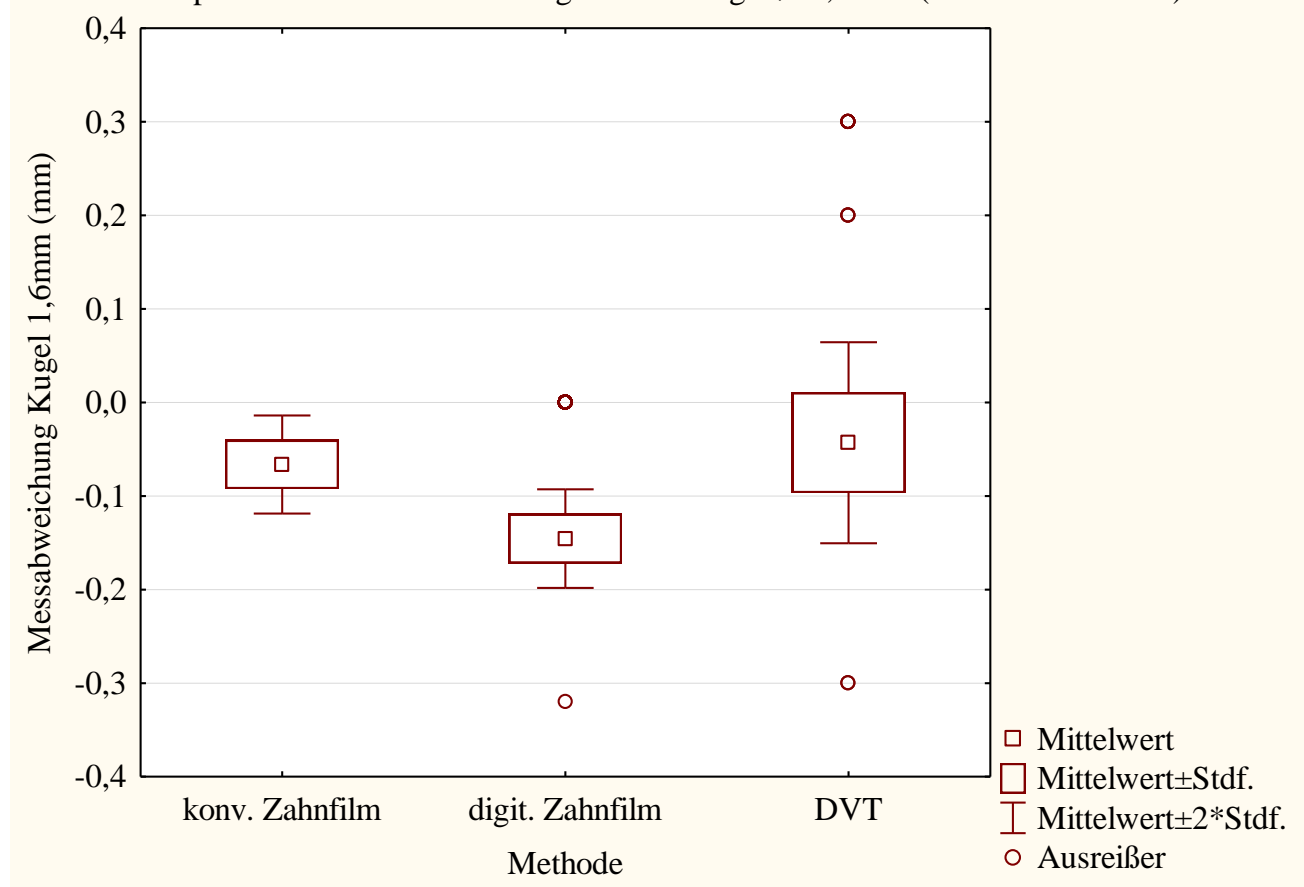

Abbildung 38: Messabweichung der Stahlkugeln Ø 1,6 mm. Die Null-Linie stellt den reellen Durchmesser laut Herstellerangabe dar. Der Mittelwert, die Box und die Whisker bilden gesondert für jede getestete Methode die Messabweichung des Stahlkugeldurchmessers ab. 


\section{Guttapercha}

Das Fremdmaterial Guttapercha wurde in Form von $1 \mathrm{~mm}$ langen und 0,3 $\mathrm{mm}$ starken Stiften (Points) in die Schweinekieferpräparate 3,12 und 13 eingebracht. In Präparat 13 waren zwei Points inseriert. In dem Prüfbogen hatten die Untersucher ein Fragment einer K-Feile als mögliches Fremdmaterial zur weiteren Auswahl. Dieser in den Präparaten nicht vorhandene Fremdkörper ähnelt den Guttaperchastiften röntgenologisch sehr und sollte den Zweck einer genauen Differenzierung zwischen zwei sehr ähnlichen Fremdkörpern haben. Die korrekte Erkennung der Guttaperchastifte ergab einen signifikanten Unterschied im intermethodischen Vergleich ( $\mathrm{p}=0,025$, Chi-Quadrat-Test). In den beiden zweidimensionalen Methoden wurden jeweils $40 \%$ der Guttapercha richtig erkannt und klassifiziert. Im konventionellen Zahnfilm wurden $10 \%$ der erkannten Fremdkörper als Fragment einer K-Feile fehldiagnostiziert und in $50 \%$ wurde die Guttapercha vom Untersucher nicht erkannt. In den digitalen Zahnfilmaufnahmen wurden $17 \%$ der erkannten Fremdkörper als Fragment einer K-Feile fehldiagnostiziert, $43 \%$ wurden nicht erkannt (Abb. 39-41). In den DVT-Aufnahmen wurden $10 \%$ korrekt als Guttapercha klassifiziert, $83 \%$ wurden nicht erkannt und $7 \%$ wurden als andere Fremdkörper fehldiagnostiziert (Abb. 42).
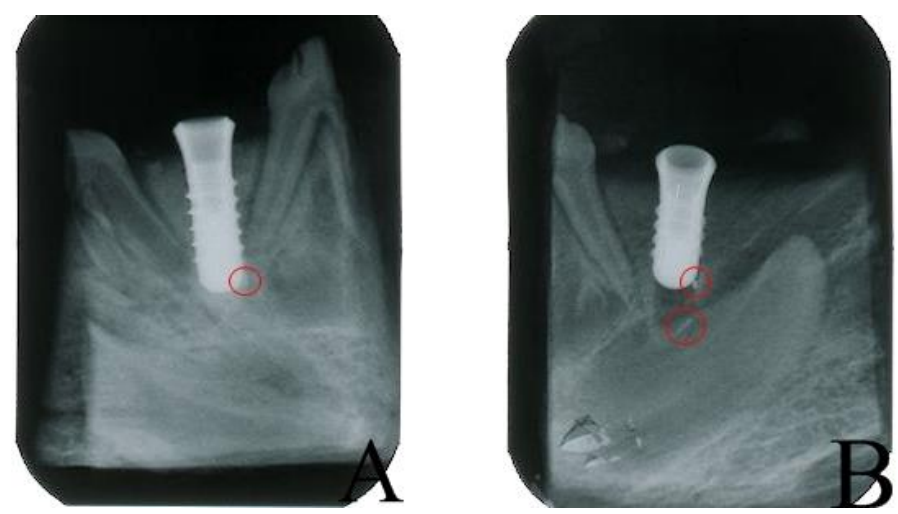

Abbildung 39: konventionelle Zahnfilmaufnahmen, Fremdmaterial Guttaperchastifte (siehe rote Umkreisung) A: Präparat 12, 1 Guttaperchastift vorhanden B: Präparat 13, zwei Guttaperchastifte vorhanden 

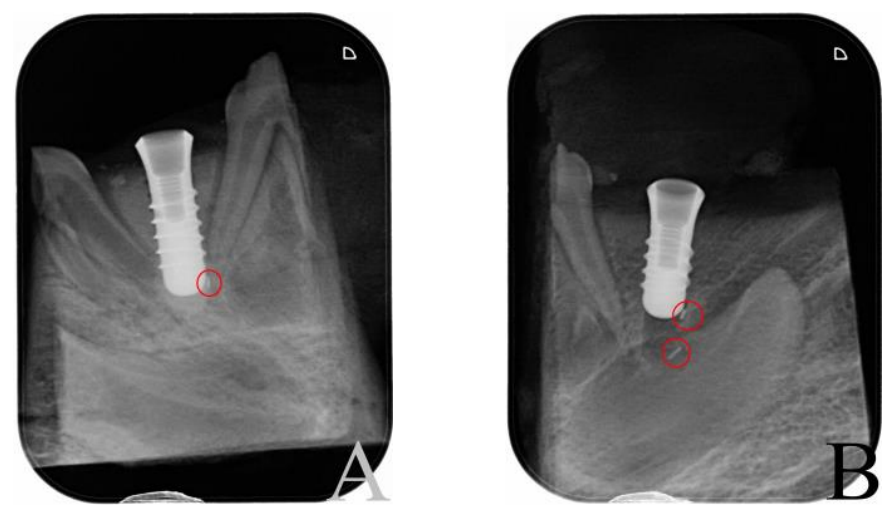

Abbildung 40: digitale Zahnfilmaufnahmen, Fremdmaterial Guttaperchastifte (siehe rote Umrandung) A: Präparat 12, ein Guttaperchastift vorhanden B: Präparat 13, zwei Guttaperchastifte vorhanden
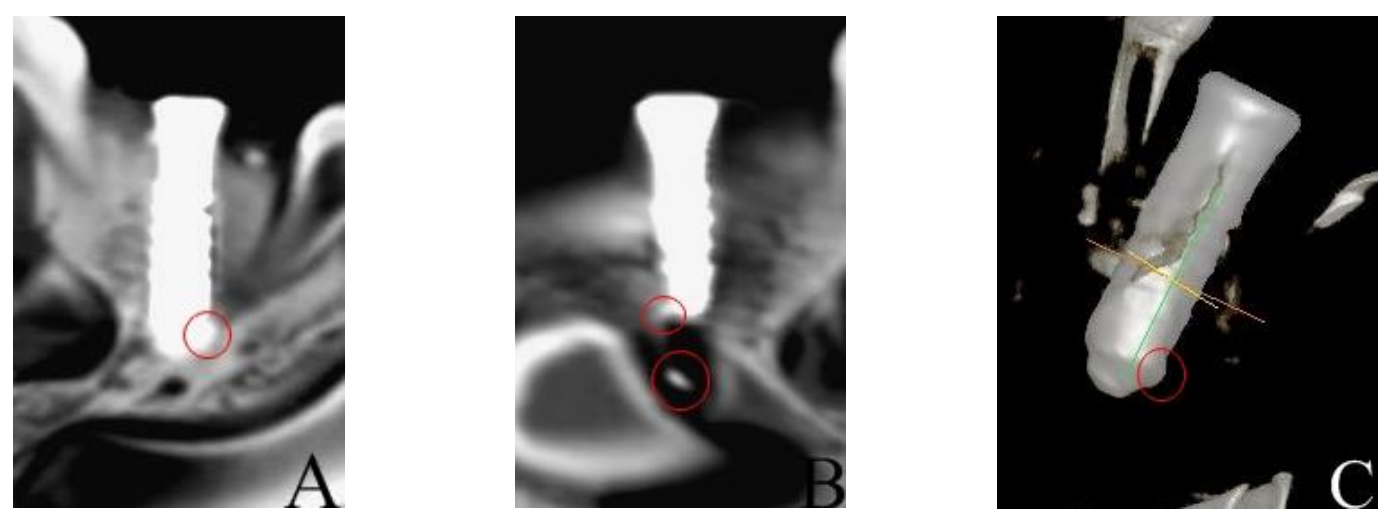

Abbildung 41: Ausschnitte aus DVT-Aufnahmen, Fremdmaterial Guttaperchastifte (siehe rote Umrandung) A: Präparat 12, ein Guttaperchastift vorhanden (koronaler Schnitt) B: Präparat 13, zwei Guttaperchastifte vorhanden (koronaler Schnitt) C: Präparat 12, Ausschnitt aus DVT, 3D-Darstellungsoption, ein Guttaperchastift vorhanden 


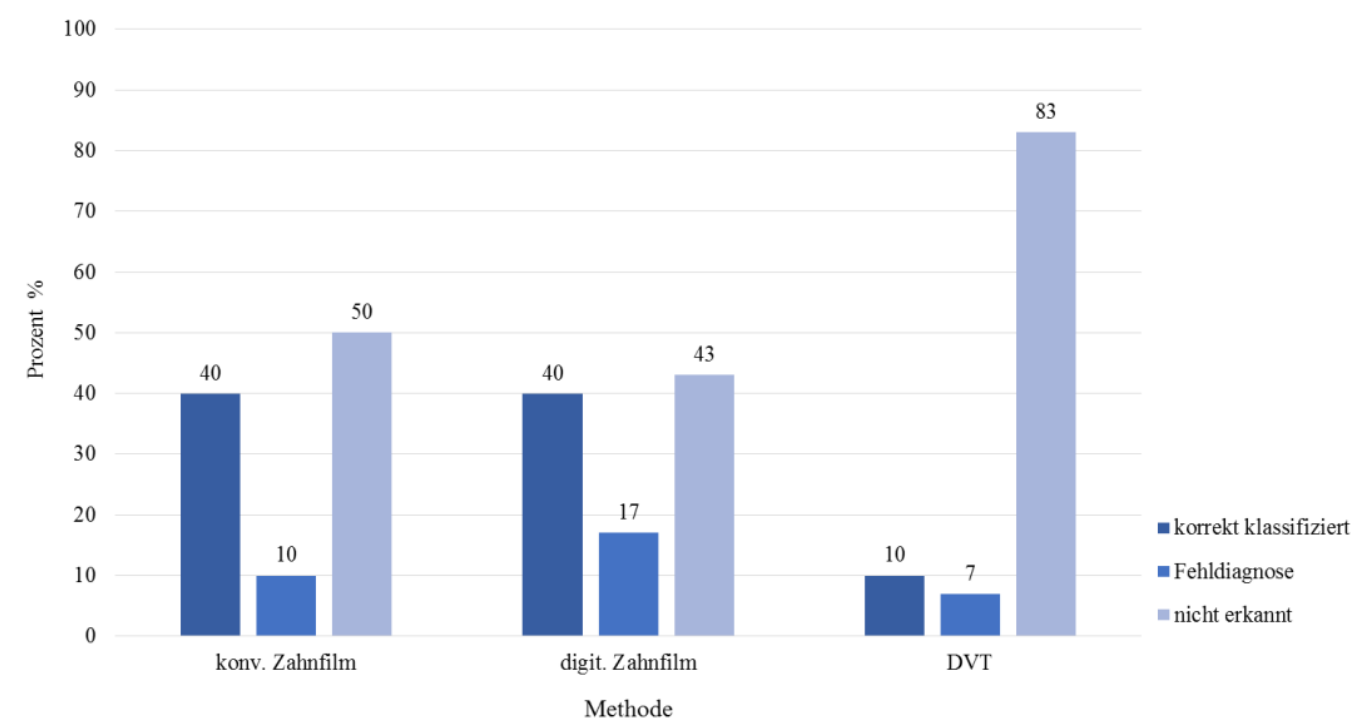

Abbildung 42: Dargestellt werden die drei Parameter korrekt klassifizierte Guttaperchastifte, nicht erkannte Guttaperchastifte und falsch diagnostizierte Fremdkörper. Es wird der prozentuale Anteil der drei Parameter von den richtig erkannten Fremdkörpern abgebildet. Jeder dieser Parameter wird durch einen Balken graphisch dargestellt. In dem Diagramm werden diese in den drei Röntgenmethoden miteinander verglichen.

\section{Beta-Tri-Calcium-Phosphat (B-TCP)}

Das Fremdmaterial Beta-Tri-Calcium-Phosphat ist ein Knochenersatzmaterial von geringer röntgenologischer Opazität. Es wurde in Form eines Granulats der Körnung 500-1000 $\mu$ m vor Insertion der Implantate mit Hilfe eines Löffels nach Hemmingway in die vorgebohrte Kavität der Präparate 3 und 12 eingebracht. Das Auffinden dieses Fremdkörpers war in allen Aufnahmen nur eingeschränkt möglich. Dabei wurde bis auf einen Fall nur das B-TCP in Präparat 12 erkannt. Die korrekte Klassifizierung des ß-TCP ergab im Methodenvergleich keinen signifikanten Unterschied $(\mathrm{p}=0,412$; Chi-Quadrat-Test). In den beiden zweidimensionalen Methoden wurde dieses Fremdmaterial jeweils zu $25 \%$ korrekt diagnostiziert. In $75 \%$ wurde es nicht detektiert. In den DVT-Aufnahmen wurde das B-TCP in $90 \%$ nicht erkannt und in $10 \%$ korrekt klassifiziert (Abb. 43). 


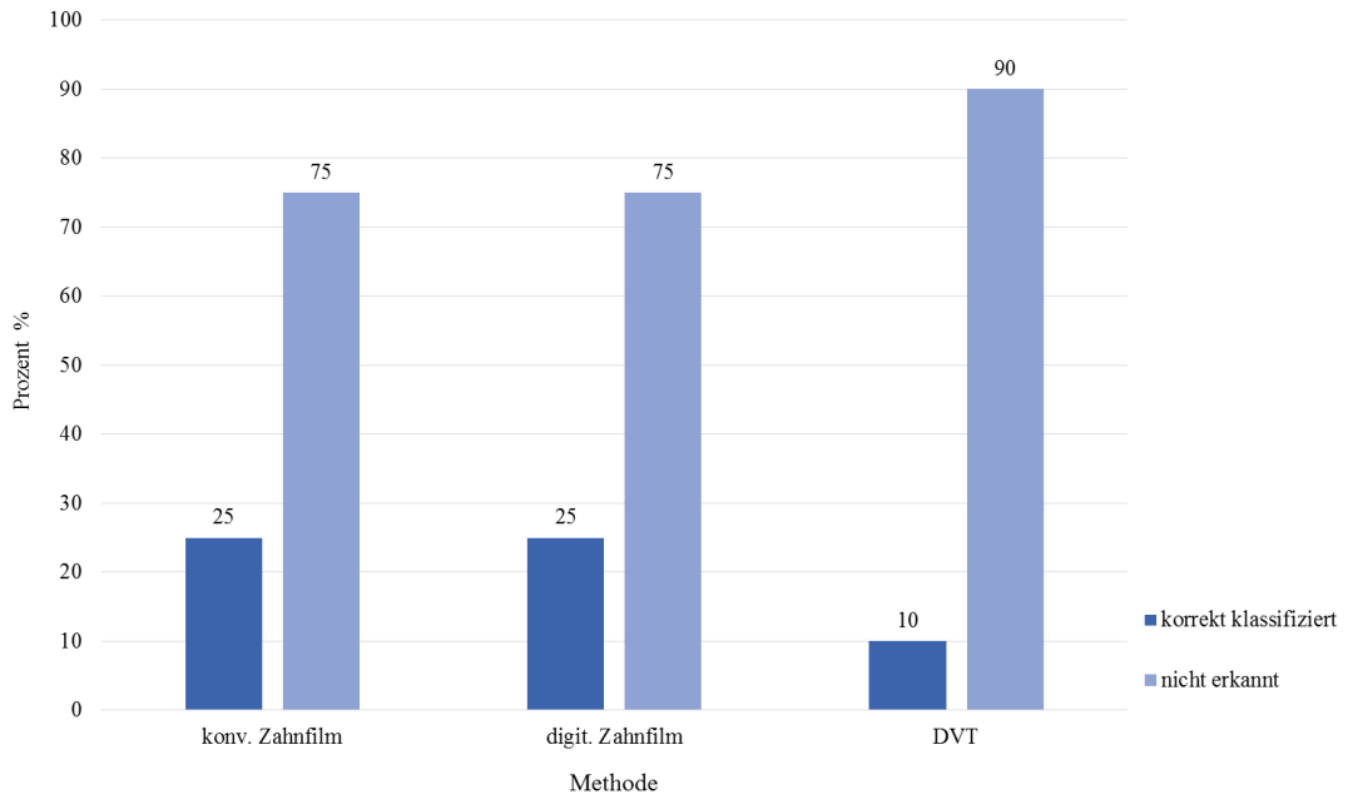

Abbildung 43: Dargestellt sind die beiden Parameter ß-TCP korrekt klassifiziert und B-TCP nicht erkannt. Sie werden durch zwei Balken beschrieben, die den prozentualen Anteil des korrekt diagnostizierten und nicht erkannten $\beta$-TCP von den richtig erkannten Fremdkörpern abbildet. In diesem Diagramm werden die Befunde in den drei Röntgenmethoden miteinander verglichen.
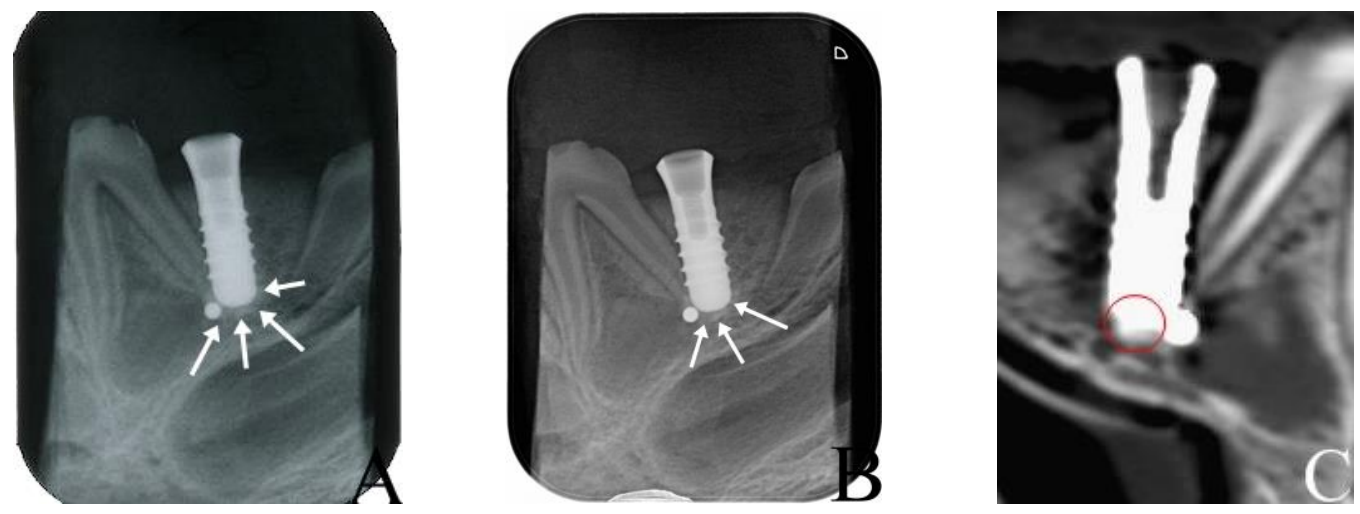

Abbildung 44: Fremdmaterial ß-TCP, Präparat 3(siehe Pfeile in A und B, C rote Umrandung) A: konv. ZF B: dig. ZF C: Ausschnitt aus DVT 

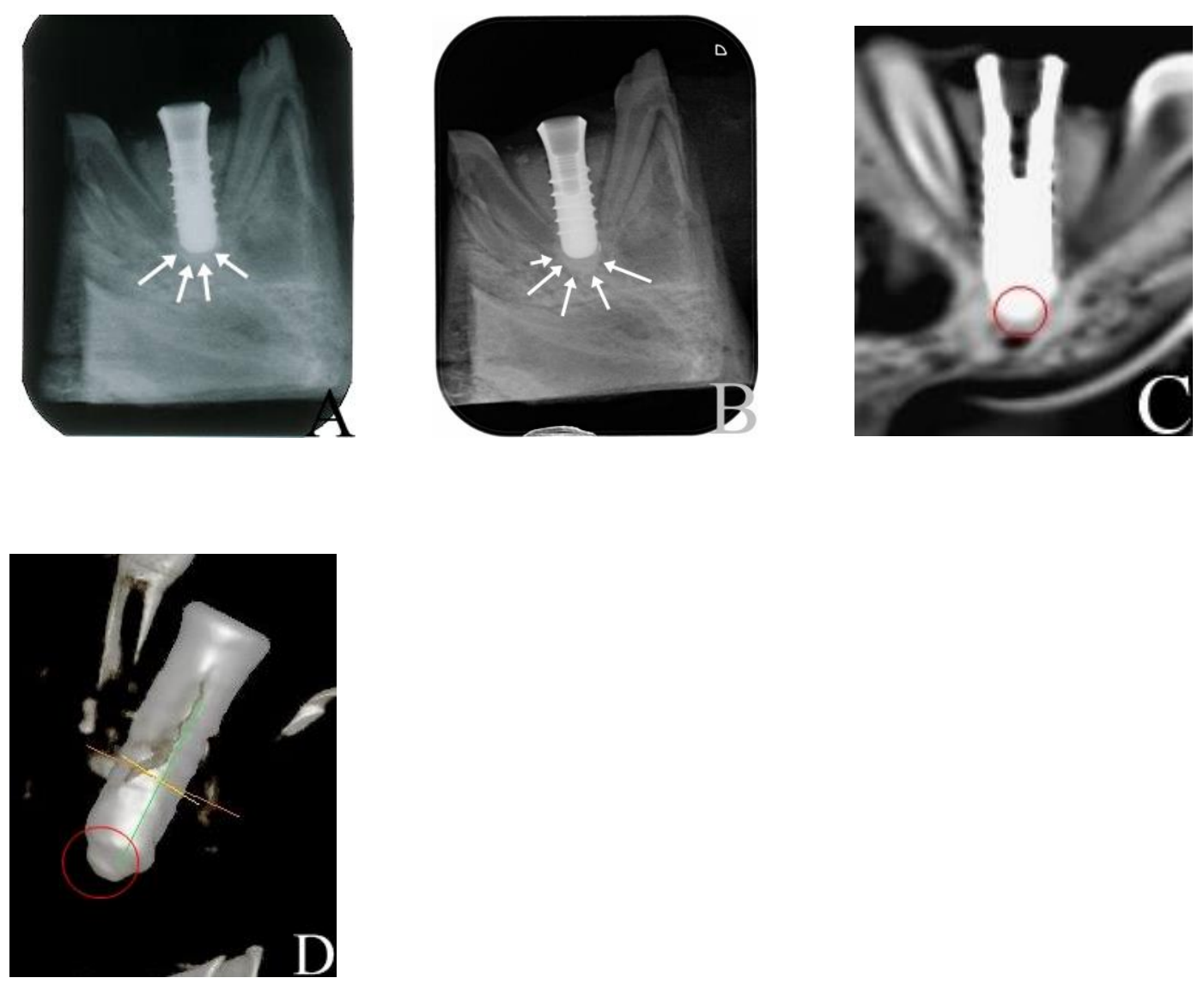

Abbildung 45: Fremdmaterial ß-TCP, Präparat 12 (siehe Pfeile in A und B, C und D rote Umrandung) A: konv. ZF B: dig. ZF C: Ausschnitt aus DVT D: Ausschnitt aus DVT, 3D-Darstellungsoption 


\subsubsection{Beeinträchtigung der Bildauswertung durch}

\section{Aufhärtungsartefakte}

In den zweidimensionalen Aufnahmemethoden konventioneller und digitaler Zahnfilm kam es zu keinen Artefaktbildungen, die eine Auswertung der Bilder hätte beeinflussen können.

Im Rahmen der DVT-Aufnahmen gaben die Untersucher bei $70 \%$ der Datensätze ein Vorliegen von Aufhärtungsartefakten an. In $80 \%$ beeinflusste dies die Bildauswertung negativ.
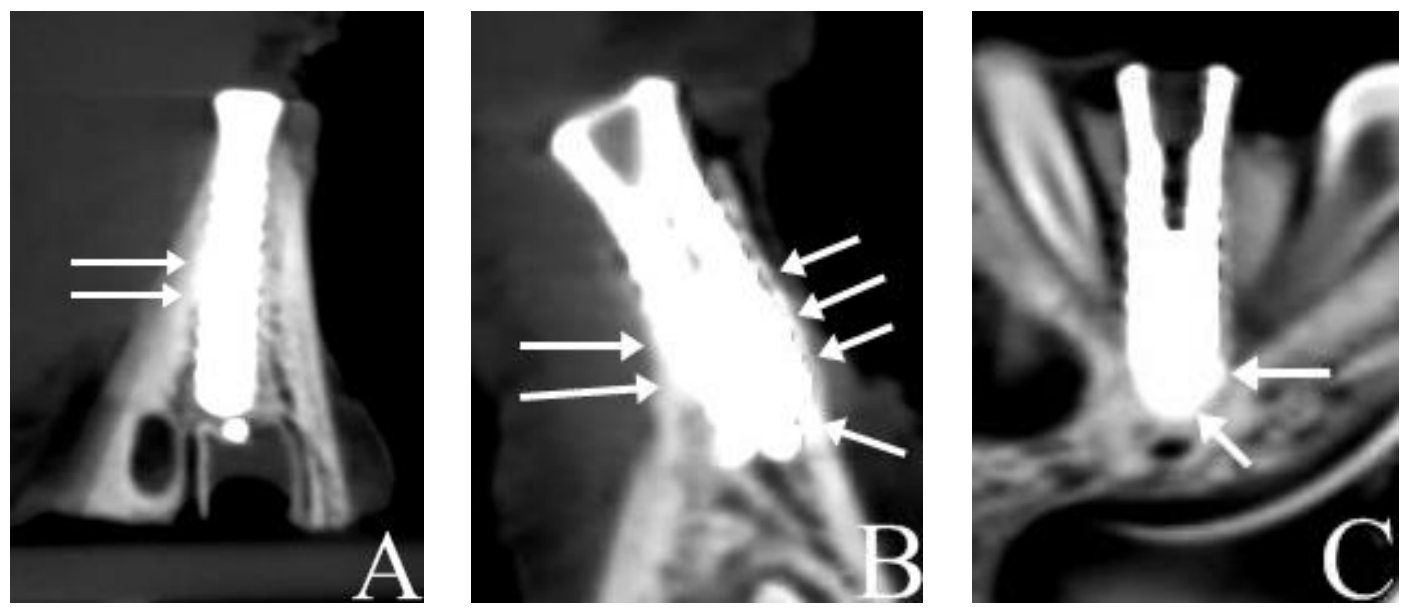

Abbildung 46: Beispiele für Bildartefakte im Bereich der metallischen Körper in DVT-Aufnahmen (siehe Pfeile) A: Präparat 4 (sagittaler Schnitt) B: Präparat 10 (sagittaler Schnitt) C: Präparat 12 (koronaler Schnitt) 


\subsection{Zweidimensionale versus dreidimensionale Verfahren}

\subsubsection{Alveolardefekte}

Sowohl in den zweidimensionalen Aufnahmen, als auch in den dreidimensionalen DVTAufnahmen konnten die dargestellten Knochendefekte differenziert werden. $65 \%$ der vorhandenen Knochendefekte konnten in den zweidimensionalen Verfahren richtig erkannt werden. In der DVT wurden 50 \% der vorhandenen Defekte richtig wahrgenommen (Abb. 47).

Bei Knochengeweben ohne Defekt konnte in allen Methoden häufiger die Unversehrtheit des Knochens korrekt erkannt werden. In $83 \%$ wurde das Knochengewebe im konventionellen und digitalen Zahnfilm korrekt als unbeschädigt beurteilt. In den DVT-Aufnahmen fand in $54 \%$ eine korrekt Beurteilung des intakten Gewebes statt (Abb. 48).

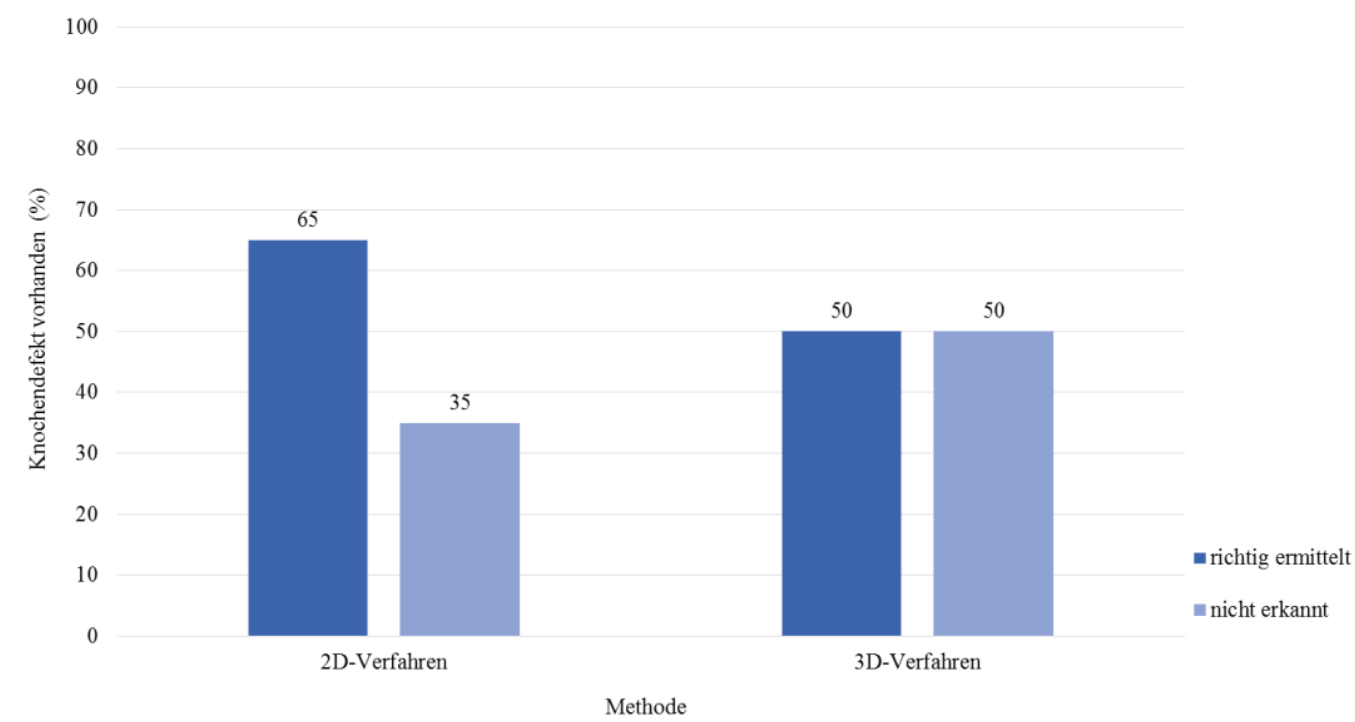

Abbildung 47: Vorhandene Knochendefekte: Dargestellt werden die beiden Parameter ,richtig ermittelter Knochendefekt " und ,nicht erkannter Knochendefekt' als prozentualer Anteil der vorhandenen Defekte in den Schweinekieferpräparaten. In diesem Diagramm werden die zweidimensionalen Methoden zusammengefasst und mit der DVT gegenübergestellt. 


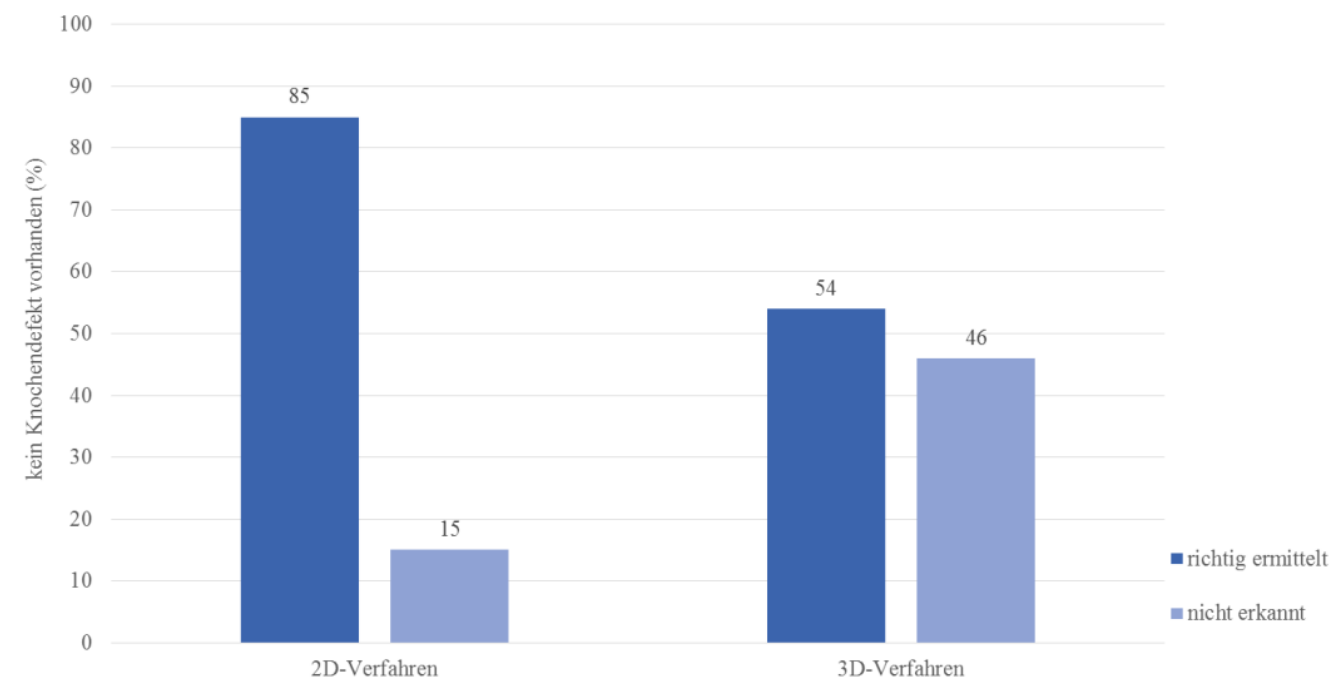

Abbildung 48: Nicht vorhandene Knochendefekte: Dargestellt werden die beiden Parameter richtiges Erkennen von unversehrtem Gewebe (richtig ermittelt) und falsches Erkennen eines Knochendefektes (nicht erkannt). Die Parameter werden als prozentualer Anteil der vorhandenen Knochendefekte in den Schweinekieferpräparaten ausgedrückt. In diesem Diagramm wird ein Vergleich zwischen den zweidimensionalen Röntgenverfahren und der DVT abgebildet.

\subsubsection{Implantatdefekte}

In den beiden zweidimensionalen Aufnahmeverfahren konnten deutlich mehr Implantatdefekte richtig erkannt werden als in dem dreidimensionalen Verfahren (DVT). In den Zahnfilmaufnahmen wurden $44 \%$ der vorhandenen Implantatdefekte als ein solcher Defekt korrekt beurteilt. In der dreidimensionalen Methode DVT wurden $10 \%$ der vorhandenen Implantatdefekte richtig erkannt. In beiden Verfahrensgruppen zeigten sich häufiger nicht erkannte Implantatdefekte als richtig erkannte Defekte (Abb. 49). In den Schweinekieferpräparaten ohne Implantatdefekte konnte eine große Anzahl von korrekten Beurteilungen festgestellt werden. Die Untersucher erkannten in den zweidimensionalen Verfahren $80 \%$ der nicht beschädigten Implantate richtig. In den DVT-Aufnahmen fiel die Beurteilung der intakten Implantate in $86 \%$ korrekt aus (Abb. 50). 


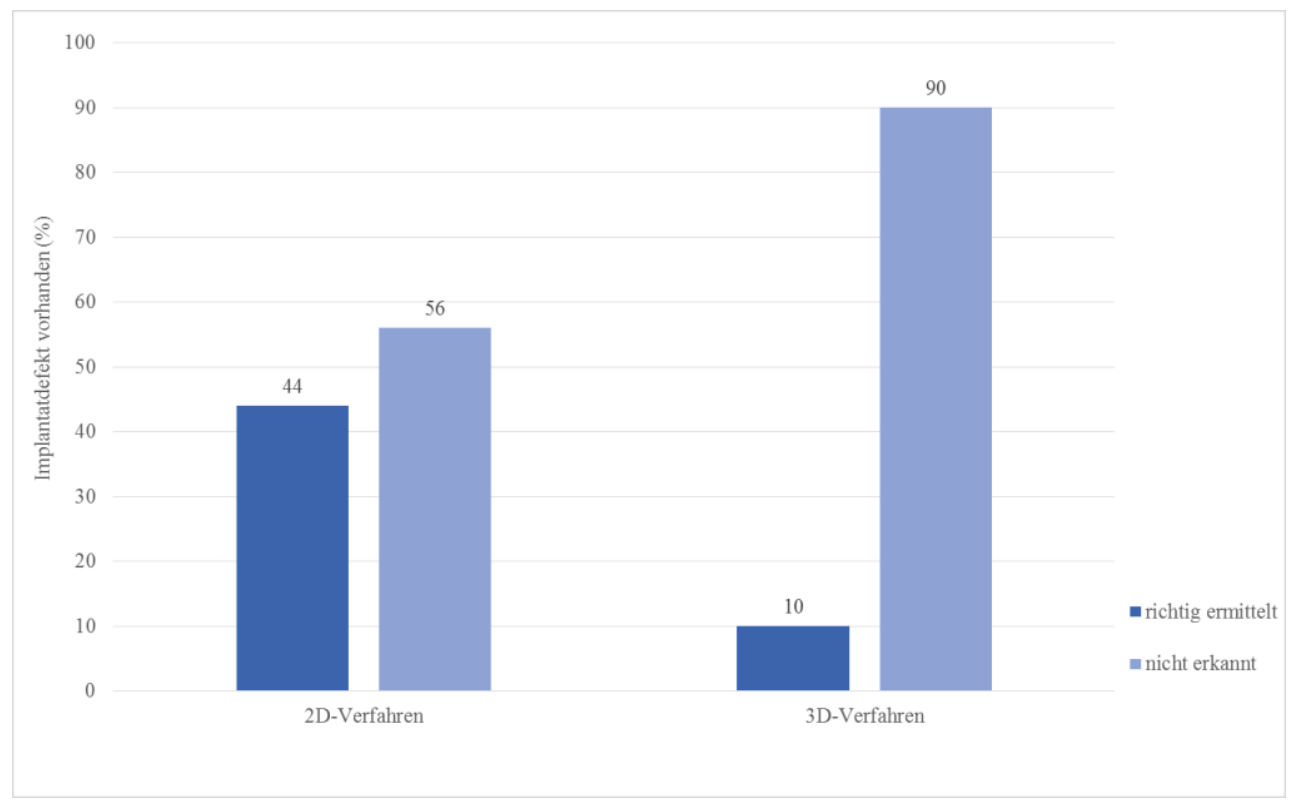

Abbildung 49: Implantatdefekt vorhanden: Dargestellt werden die beiden Parameter richtig ermittelter Implantatdefekt und nicht erkannter Implantatdefekt. Sie werden als prozentualer Anteil der vorhandenen Implantatdefekte dargestellt. Zudem werden in diesem Diagramm die Ergebnisse in den beiden Gruppen zweidimensionale und dreidimensionale Verfahren verglichen.

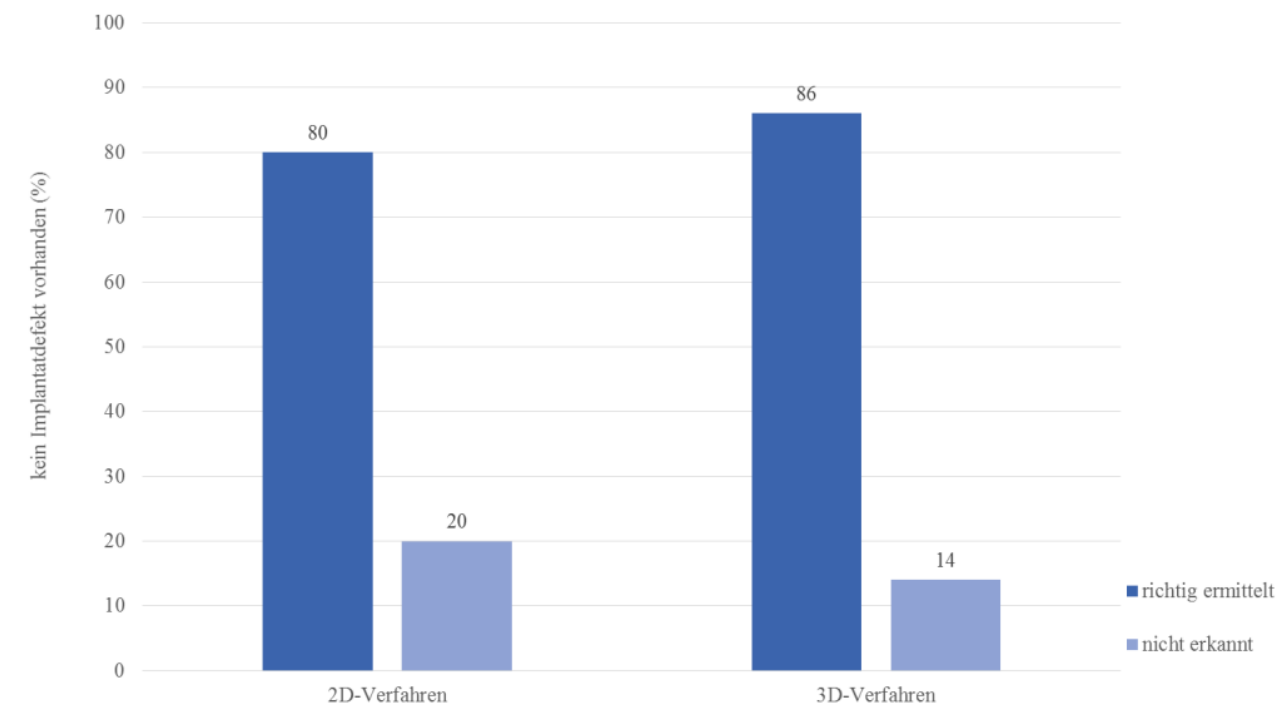

Abbildung 50: Kein Implantatdefekt: Dargestellt sind die Parameter richtiges Erkennen eines unbeschädigten Implantates (richtig erkannt) und falsches Erkennen eines Implantatdefektes (nicht erkannt). Sie werden als prozentualer Anteil der vorhandenen Implantatdefekte dargestellt. In diesem Diagramm werden die Ergebnisse der zweidimensionalen Methoden mit denen der dreidimensionalen Methode verglichen. 


\subsubsection{Fremdkörper}

Die Fremdkörper konnten in allen Methoden gut dargestellt werden und wurden sehr häufig in allen Aufnahmen richtig erkannt. In den zweidimensionalen Verfahren wurden $97 \%$ der vorhandenen Fremdkörper richtig erkannt. In der dreidimensionalen Methode DVT erkannten die Untersucher $81 \%$ der inserierten Fremdkörper (Abb. 51). War kein Fremdkörper vorhanden, konnte das intakte Gewebe um das Implantat differenziert beurteilt werden und das Vorhandensein eines Fremdkörpers ausgeschlossen werden. Dabei beurteilten die Untersucher in den zweidimensionalen Verfahren Gewebe ohne Fremdkörper in 97 \% richtig. In der DVT wurden $100 \%$ des gesunden Gewebes auch als solches beurteilt (Abb. 52).

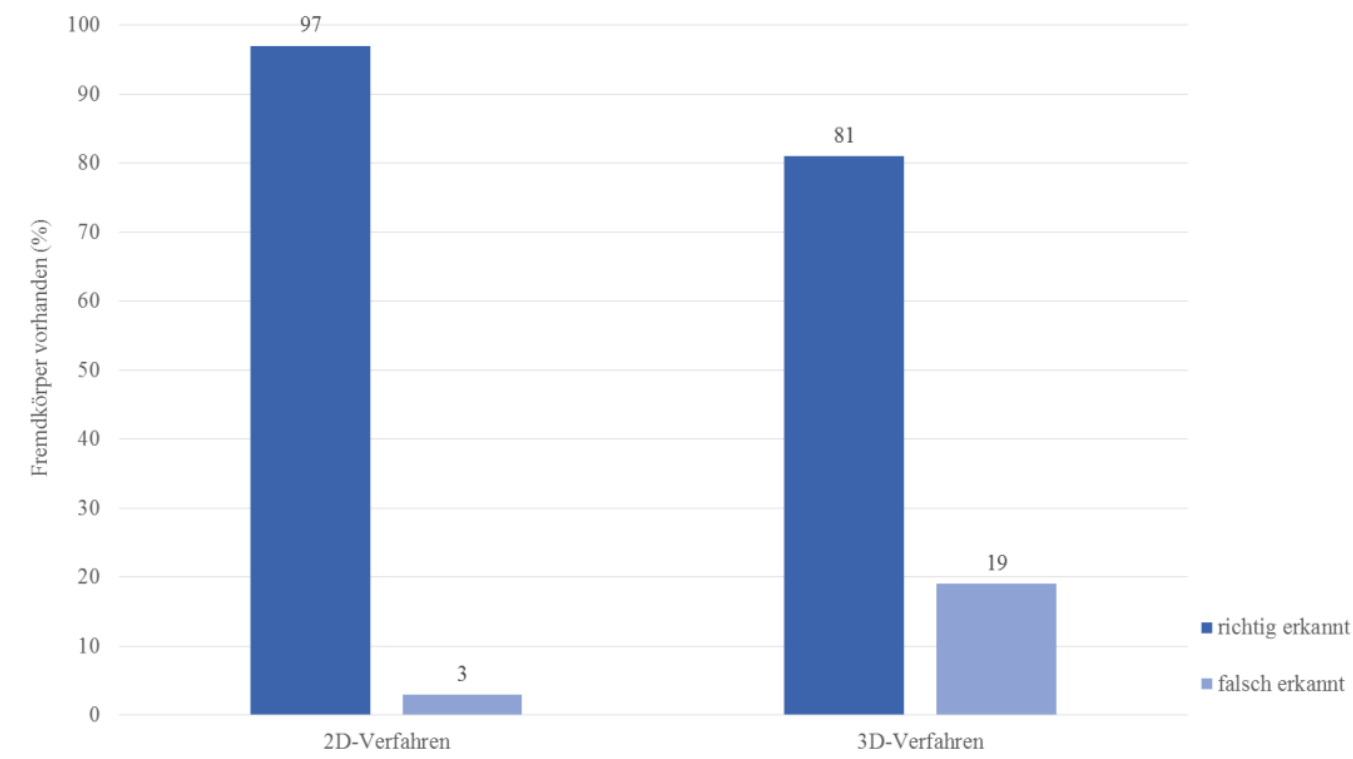

Abbildung 51: Fremdkörper vorhanden: Dargestellt sind die Parameter richtig erkanntes Vorhandensein eines Fremdkörpers (richtig erkannt) und nicht erkannter Fremdkörper (nicht erkannt). Die Werte der Balken im Diagramm geben den prozentualen Anteil der vorhandenen Fremdkörper in den Präparaten wieder. Der Grafik ist außerdem ein Vergleich der Ergebnisse von zweidimensionalen und dreidimensionalen Verfahren zu entnehmen. 


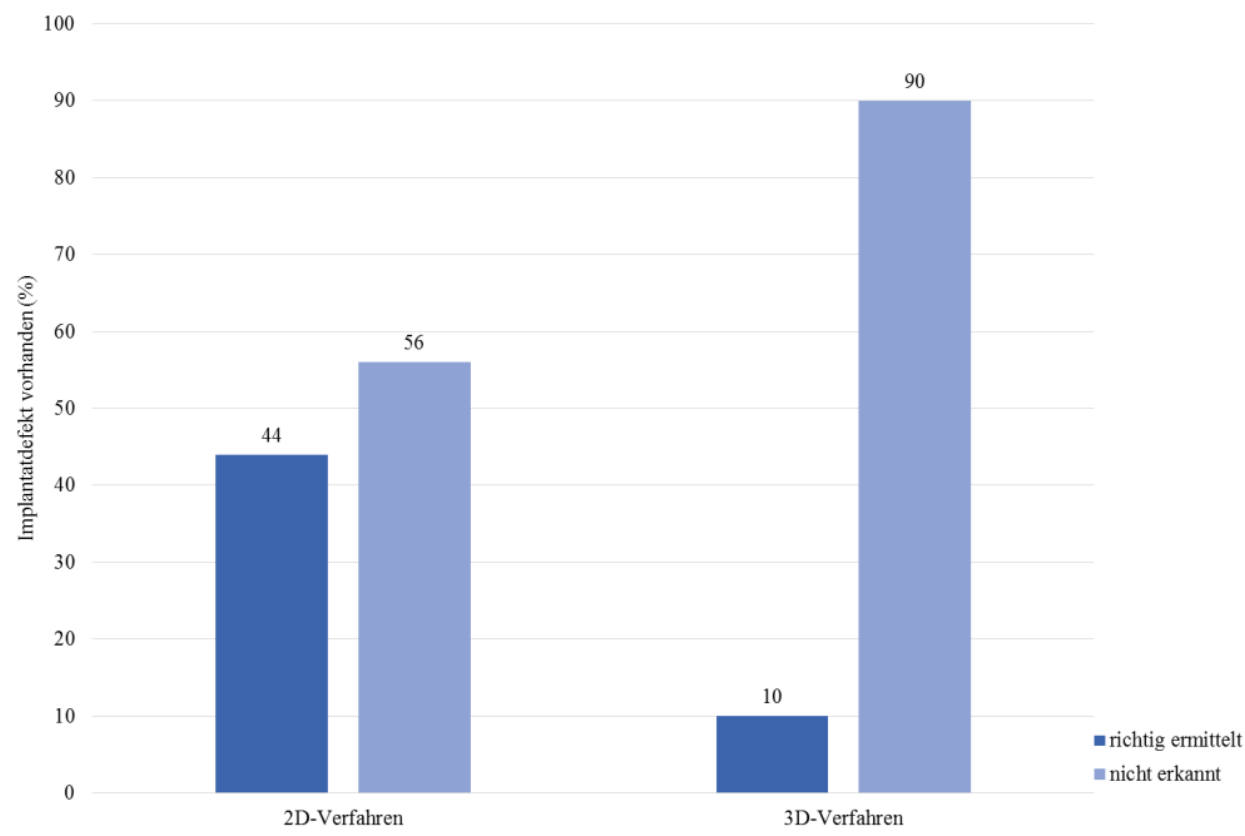

Abbildung 52: Kein Fremdkörper vorhanden: Dargestellt sind die beiden Parameter richtiges Erkennen eines fehlenden Fremdkörpers im Präparat (richtig erkannt) und das falsche Erkennen eines Fremdkörpers (falsch erkannt). Die Werte der Parameter sind als prozentualer Anteil der vorhandenen Fremdkörper in den Präparaten angegeben. Zudem werden in dieser Grafik die Ergebnisse der zweidimensionalen und dreidimensionalen Verfahren gegenübergestellt. 


\subsection{Vergleich der Untersucher}

Der Vergleich der Untersucher wurde hinsichtlich der Messpräzision der Implantatlängen und -durchmesser durchgeführt. Darüber hinaus wurden die Untersucher in der korrekten Klassifizierung der Knochen- und Implantatdefekte sowie der Fremdkörperklassifizierung beurteilt. Es ergab sich nur bei der Messpräzision der Implantatdurchmesser ein signifikanter Unterschied zwischen den Untersuchern ( $\mathrm{p}<0,0001$; F-Test) (Abb. 53).

Boxplot: Untersuchervergleich bezüglich der Messpräzision der Implantatdurchmesser (mm)

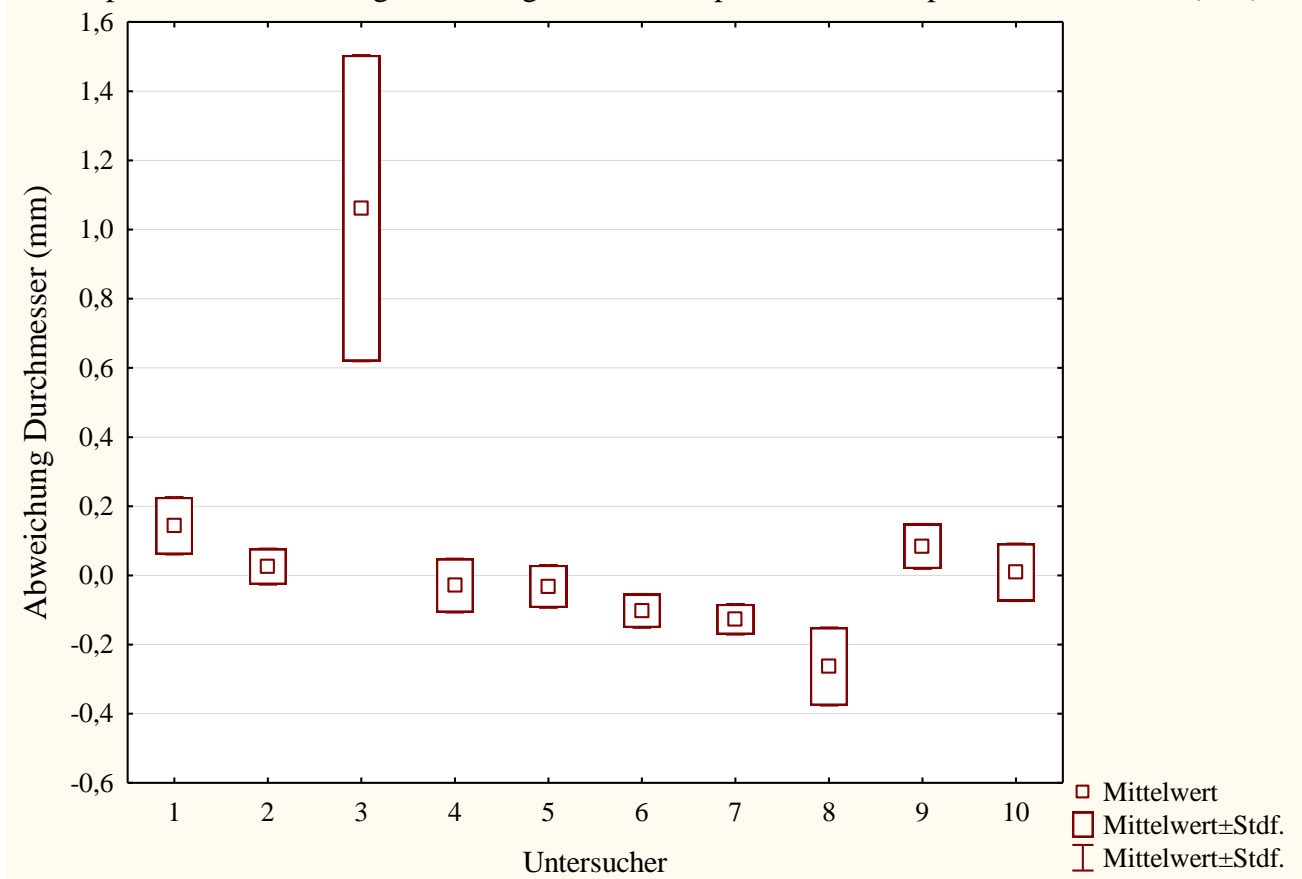

Abbildung 53: Dargestellt ist der Vergleich der Untersucher bezüglich der Messpräzision für die Implantatdurchmesser. Für jeden Untersucher mit den Ordnungsnummern 1-10 sind die Mittelwerte der Messabweichungen in mm aufgetragen. Mittelwert und Standardfehler zeigen die Bereiche der

Messabweichungen für jeden Untersucher und lassen erkennen, ob ein Untersucher tendenziell im positiven oder negativen Bereich der Messabweichungen liegt, sowie die Präzision und Streuung der Messungen der einzelnen Untersucher. 
In Abb. 53 ist zu erkennen, dass eine signifikante Messabweichung innerhalb der Untersuchergruppe durch Untersucher 3 begründet vorliegt. Die Messergebnisse zeigen eine geringe Präzision und eine weite Streuung. Im Vergleich zeigen die anderen neun Untersucher ähnliche Messabweichungen ohne signifikante Unterschiede. Die weiteren Vergleiche in der Untersuchergruppe ermittelten keine signifikanten Unterschiede. Sie werden im Folgenden anhand von Grafiken dargestellt (Abb. 54-57). Die Messung der Implantatlängen war ohne Signifikanz ( $\mathrm{p}=0,25 ;$ F-Test). Die korrekte Klassifizierung der Alveolardefekte, Implantatdefekte und der Fremdmaterialen zeigten keinen signifikanten Unterschied innerhalb der Untersuchergruppe (Alveolardefekte: $\mathrm{p}=0,68$; Chi-Quadrat-Test, Implantatdefekte: $\mathrm{p}=0,55$; Chi-Quadrat-Test, Fremdmaterialien: $\mathrm{p}=0,47$; Chi-Quadrat-Test).

Boxplot: Vergleich der Untersucher in Bezug auf die Messabweichung der Implantatlängen (Mittelwerte in $\mathrm{mm}$ )

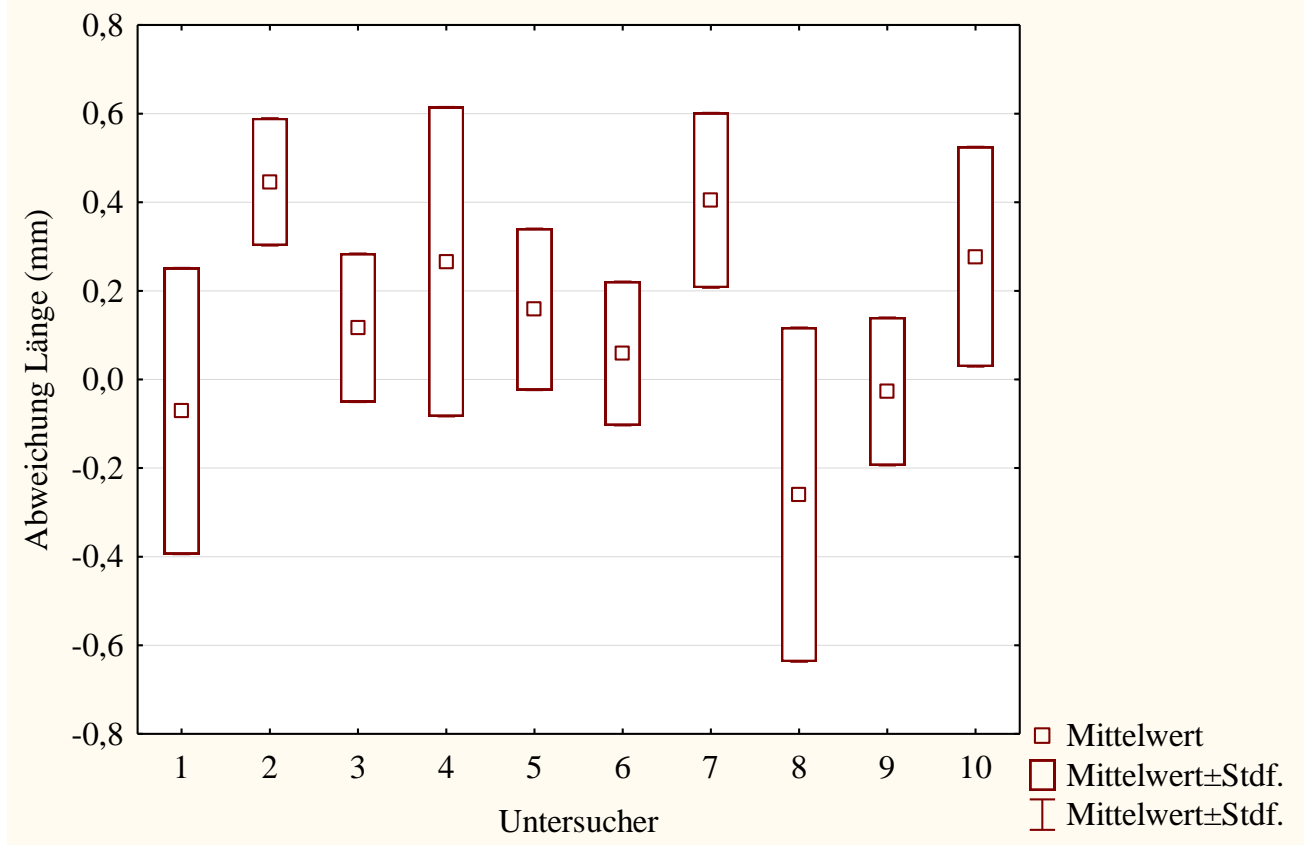

Abbildung 54: Dargestellt ist der Vergleich der Untersucher bezüglich der Messpräzision für die Implantatlängen. Für jeden Untersucher mit den Ordnungsnummern 1-10 sind die Mittelwerte der Messabweichungen in mm aufgetragen. Mittelwert und Standardfehler zeigen die Bereiche der Messabweichungen für jeden Untersucher und stellen dar, ob ein Untersucher tendenziell im positiven oder negativen Bereich der Messabweichungen liegt. 


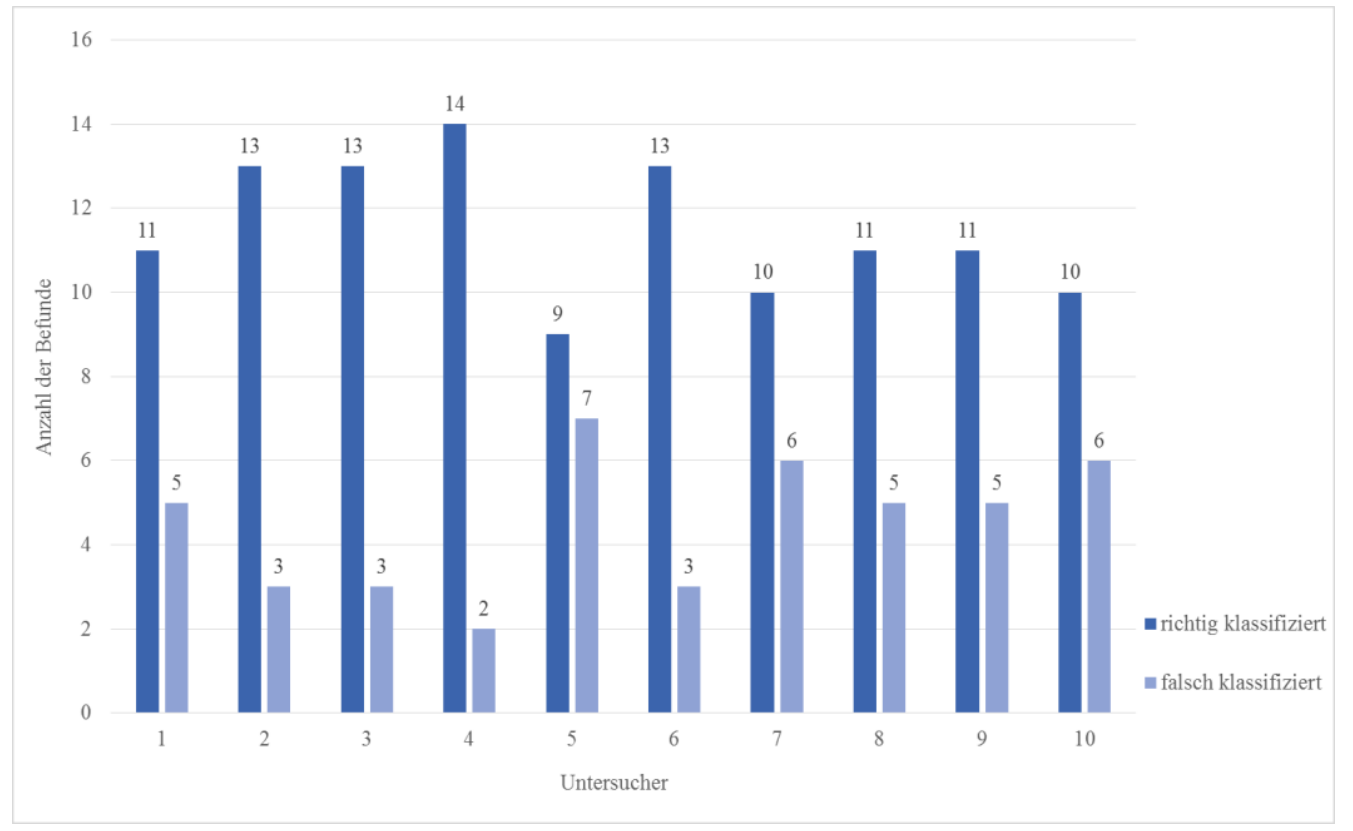

Abbildung 55: Alveolardefekte: Dargestellt ist der Vergleich der Untersucher bezüglich der korrekten Klassifizierung der Knochendefekte. Für jeden Untersucher mit den Ordnungsnummern 1-10 sind zwei Balken für richtig klassifizierte und falsch klassifizierte Defekte abgebildet. Die Balken drücken die absolute Anzahl der Befunde aus.

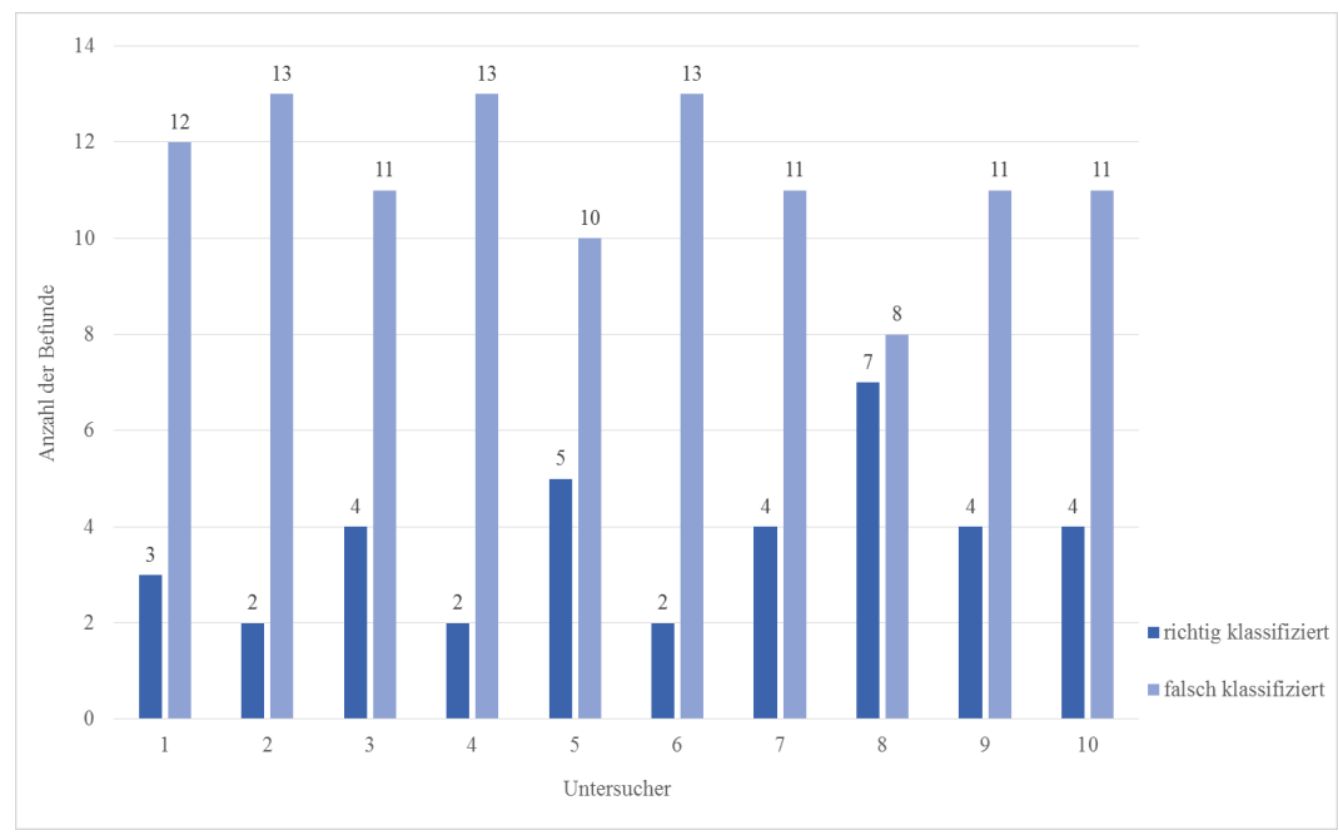

Abbildung 56: Implantatdefekte: Dargestellt ist der Vergleich der Untersucher bezüglich der korrekten Klassifizierung der Implantatdefekte. Für jeden Untersucher mit den Ordnungsnummern 1-10 sind zwei Balken für richtig klassifizierte und falsch klassifizierte Defekte abgebildet. Die Balken drücken die absolute Anzahl der Befunde aus. 


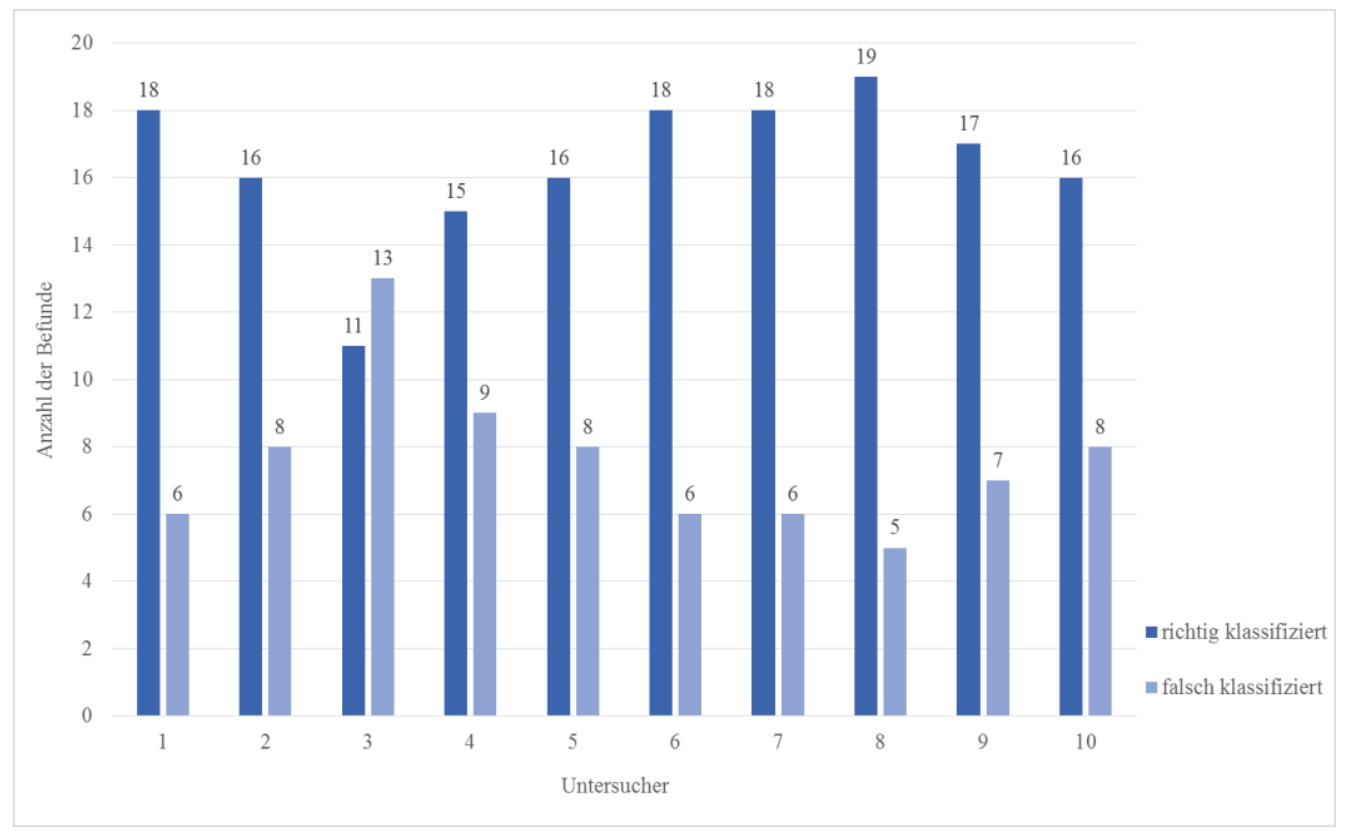

Abbildung 57: Fremdmaterialien: Dargestellt ist der Vergleich der Untersucher bezüglich der korrekten Klassifizierung der Fremdkörperarten. Für jeden Untersucher mit den Ordnungsnummern 1-10 sind zwei Balken für richtig klassifizierte und falsch klassifizierte Fremdkörper abgebildet. Die Balken drücken die absolute Anzahl der Befunde aus. 


\section{$\underline{\text { 4. Diskussion }}$}

Die Fragestellung dieser Untersuchung befasst sich mit der Evaluation von drei bildgebenden röntgenologischen Verfahren in der Zahnmedizin, bezogen auf die Darstellbarkeit von enossalen Implantaten, pathologischen Veränderungen in Form von Defekten und Fremdmaterialien sowie des umliegenden Alveolarknochens.

Zehn Schweinekieferpräparate, versehen mit unterschiedlichen Implantaten, Gewebe- und Implantatdefekten sowie inserierten Fremdmaterialien wurden radiologisch dargestellt.

Die intraorale Zahnfilmaufnahme mit dem konventionellen Filmfolien-System und dem digitalen Speicherfolien-System wurde mit der dreidimensionalen digitalen Volumentomographie (DVT) verglichen. Die noch relativ neue und ständig weiterentwickelte DVT steht zwei etablierten Röntgenverfahren der Zahnmedizin gegenüber. Die Untersuchung sollte zeigen, ob bei der Beurteilung der Röntgenaufnahmen durch eine Untersuchergruppe mit einheitlichem Wissenstand ein signifikanter Unterschied hinsichtlich der Darstellbarkeit von periimplantärem Gewebe zwischen den einzelnen Methoden besteht. In der präoperativen Behandlungsphase vor Implantation werden im Rahmen der Diagnostik unter anderem bildgebende Verfahren zur Bestimmung des Knochenangebotes und der Knochenqualität verwendet. Hierzu zählen in der Zahnmedizin zunächst der intraorale Zahnfilm und das Orthopantomogramm (OPG). Diese zweidimensionalen Röntgenverfahren sind bewährte Techniken zur Primärdiagnostik. Der intraorale Zahnfilm zeigt bei paralleler Aufnahmetechnik eine gute Detailgenauigkeit, ist aber limitiert auf einen kleinen Bereich von ca. drei Zähnen. Das OPG bietet eine gute Übersicht über den Alveolarknochen und wichtige angrenzende Strukturen wie Canalis mandibulae und Sinus maxillaris (Chan et al. 2010). In der Implantologie kann eine dreidimensionale Aufnahme notwendig sein, wenn durch vorherige klinische Untersuchung und zweidimensionale Bildgebung keine eindeutige Beurteilung der präoperativen Knochensituation möglich ist (Schulze et al. 2013). Die computergestützte dreidimensionale Navigation kann zur Herstellung von Operationsschablonen und zur virtuellen Planung der idealen Platzierung der Implantate im Kieferknochen herangezogen werden (Schulze et al. 2009). 
Die klassische Computertomographie ist derzeit der Goldstandard der dreidimensionalen Bildgebung von Hartgeweben in der Mund-, Kiefer- und Gesichtschirurgie. Die anatomischen Strukturen sowie pathologische Veränderungen werden hierbei überlagerungsfrei und exakt in Größe und Ausdehnung wiedergegeben. Durch die Kontrastgenauigkeit und hohe Bildauflösung sind einzelne Strukturen sehr gut voneinander zu differenzieren (Medelnik et al. 2011; Poeschl et al. 2013). Dagegen stehen die Kosten eines CT-Gerätes und der Betrieb durch einen Radiologen, die eine Nutzung eines solchen Gerätes nur im Klinikbetrieb sinnvoll erscheinen lässt (Ludlow und Ivanovic 2008). Patientenbezogen bedeutet eine CT-Aufnahme eine deutlich höhere Strahlenbelastung als konventionelle Bildgebungsverfahren in der Zahnmedizin (Medelnik et al. 2011). Eine Alternative zur klassischen Computertomographie wurde mit der Entwicklung der digitalen Volumentomographie in der Zahnmedizin und der Mund-, Kiefer- und Gesichtschirurgie eingeführt. Die wesentlichen Vorteile dieser dreidimensionalen Aufnahmetechnik liegen in der Auswahl zwischen unterschiedlich großen Aufnahmefeldern (field of view), einer kürzeren Aufnahmezeit und einer damit verbundenen reduzierten Strahlendosis. Durch die Nutzbarkeit in einer zahnärztlichen Praxis mit der entsprechend erworbenen Fachkunde und durch die geringeren Kosten als die eines CTGerätes ist die DVT-Technik in den vergangenen Jahren stark entwickelt und vermarktet worden (Scarfe 2006).

In mehreren Studien wurde die Bildauflösung und die exakte Messung von linearen Werten im MSCT und im DVT evaluiert (Suomalainen et al. 2008; Al-Ekrish und Ekram 2011; Damstra et al. 2010; Kobayashi et al. 2004). Sie kommen zu dem Ergebnis, dass mit der DVTTechnik eine exakte Messung des Knochenangebotes möglich ist und eine zuverlässige Implantatplanung erfolgen kann. In einer Untersuchung von Al-Ekrish et al. war die digitale Volumentomographie im Vergleich mit einem CT die genauere Methode bei der präoperativen Messung des Knochenangebotes vor Implantation (Al-Ekrish und Ekram 2011). Auf Grund der Unterschiede der einzelnen Methoden wurde in der aktuellen Studie ein Prüfbogen erstellt, anhand dessen die Aufnahmen der einzelnen Methoden beurteilt wurden und in einem intermethodischen Vergleich gegenübergestellt werden konnten. Die Untersucher dieser Studie gehörten dem dritten Semester des klinischen Studienabschnittes an und hatten einen einheitlichen Wissensstand. 
Im Gegensatz zur Untersuchergruppe der Studie von Jarnot 2011 ergab sich eine homogene Klientel mit gleichem Vorwissen. In der vorherigen Untersuchung unterschieden sich die Fachgebiete der Untersucher. Zwei Oralchirurgen und ein Radiologe evaluierten die Röntgenaufnahmen. Somit hatten sie unterschiedliche Erfahrungswerte mit den untersuchten Methoden und unterschiedliche Wissensstände. Als nachteilig an der aktuellen Studie kann die mangelnde klinische Erfahrung der Studienteilnehmer gewertet werden. Zwar bestand zum Zeitpunkt der Evaluation ein gleicher Wissensstand, jedoch ebenfalls ein geringer Erfahrungswert mit beispielsweise der DVT-Technik. Im Hinblick auf die Untersuchung kann die fehlende Erfahrung aber auch als ein Faktor gewertet werden, der zur Konzentration auf die einzelnen Röntgenmethoden führt. Somit wurden die Röntgenaufnahmen ohne Beeinflussung von Erfahrungswerten evaluiert.

Die unterschiedlich dichten Hartgewebe Spongiosa und Kompakta sowie Schmelz und Dentin konnten in den drei röntgenologischen Methoden gut voneinander differenziert werden. Der weniger starke Kontrast zwischen Spongiosa und Kompakta wurde deutlich besser in den DVT-Aufnahmen differenziert, der Kontrast zwischen Schmelz und Dentin konnte in den zweidimensionalen Methoden eindeutiger unterschieden werden. Am häufigsten gelang dies in den digitalen Zahnfilmaufnahmen.

Die metrischen Distanzmessungen (Implantatlänge und -durchmesser) zeigten eine intermethodische Signifikanz. Die DVT und die konventionellen Zahnfilmaufnahmen ergaben ähnliche Messabweichungen. In den digitalen Zahnfilmaufnahmen zeigten sich größere Messdifferenzen, insbesondere bei der Längenbestimmung der Implantate kam es zu deutlichen Abweichungen. Die Messungen in dieser Untersuchung wurden nur dann als korrekt einbezogen, wenn sie maximal um $0,1 \mathrm{~mm}$ von der Herstellerangabe abwichen. Alle Längenmessungen der Implantate in den digitalen Zahnfilmaufnahmen hatten eine größere Differenz zu den Herstellerangaben. Die Durchmesserbestimmungen in den digitalen Zahnfilmaufnahmen erfüllten die Vorrausetzungen von einer maximalen Messabweichung von $0,1 \mathrm{~mm}$ von der Herstellerangabe nur in $7 \%$. Möglicherweise waren diese Bedingungen zu stark eingegrenzt. In einer Untersuchung von Vandenberghe et al. wurde eine Abweichung von $0,56 \mathrm{~mm}$ in digitalen intraoralen Zahnfilmaufnahmen gemessen (Vandenberghe et al. 2008). 
$\mathrm{Zu}$ ähnlichen Ergebnissen kamen Scarfe et al. bei vertikalen Messungen in digitalen und konventionellen Zahnfilmaufnahmen ergaben sich ebenfalls größere Messabweichungen als 0,1 mm (Scarfe et al. 1999). Im Vergleich mit den Ergebnissen der Messabweichungen von der Untersuchung von Jarnot, in der dieselben Präparate verwendet wurden, wurde der digitale Zahnfilm nicht untersucht (Jarnot 2011). Daher können nur die Ergebnisse der Messabweichungen für den konventionellen Zahnfilm und die DVT gegenübergestellt werden. Die Ergebnisse der Messabweichungen für die DVT unterscheiden sich in beiden Untersuchungen gering voneinander (Mittelwerte der Messabweichuchung Implantatlängen im DVT: Jarnot: $+0,51 \mathrm{~mm}$, aktuelle Untersuchung: $+0,61 \mathrm{~mm}$; Mittelwerte der Implantatdurchmesser: Jarnot: $+0,02 \mathrm{~mm}$, aktuelle Untersuchung: $-0,05 \mathrm{~mm}$ ). Die Ergebnisse der Messabweichungen der konventionellen Zahnfilmaufnahmen unterschieden sich in der aktuellen Untersuchung deutlich von den Ergebnissen der Messabweichungen von Jarnot (Mittelwerte der Messabweichung Implantatlängen im konventionellen Zahnfilm: Jarnot: -0,18 mm, aktuelle Untersuchung: $+0,43 \mathrm{~mm}$; Mittelwerte der Implantatdurchmesser: Jarnot: $-0,125 \mathrm{~mm}$, aktuelle Untersuchung: $+0,61 \mathrm{~mm}$ ). Einerseits arbeiteten in den beiden Studien unterschiedliche Untersuchergruppen, andererseits waren die Gruppen in differierender Weise auf die Messungen der Implantate vorbereitet. Den Untersuchern der aktuellen Studie waren die Herstellerangaben der Implantatlängen und -durchmesser nicht bekannt. Dementsprechend wurden die Messungen von den Untersuchern unbeeinflusst von Richtwerten vorgenommen. In der Untersuchung von Jarnot wurden die Untersucher über die möglichen Zielgrößen der Implantate vor der Evaluation in Kenntnis gesetzt (Jarnot 2011). Die aktuellen Ergebnisse der Evaluation der iatrogen produzierten Alveolardefekte unterschieden sich in den drei röntgenologischen Methoden nicht signifikant voneinander. In den zweidimensionalen Methoden wurden mehr Defekte von den Untersuchern erkannt als in der DVT. In der qualitativen Evaluation, d.h. in der korrekten Klassifizierung eines Defektes nach dem Erkennen bzw. Auffinden, zeigte sich jedoch die DVT als überlegen. Der Knochendefekt in Präparat 14 (Ersatz, lingual perforiert) wurde nur in der dreidimensionalen Aufnahme korrekt klassifiziert. In den zweidimensionalen Aufnahmen wurde er ebenfalls erkannt, wurde aber fälschlicherweise der Defektklasse „Überdimension“ oder „Längsrille“ zugeordnet. Die linguale Perforation konnte nur in der DVT-Aufnahme dargestellt werden. 
Demnach ist die intraorale Zahnfilmaufnahme eine hochauflösende Röntgentechnik, nach dem Erkennen eines Knochendefektes ist eine weitere Diagnostik durch überlagerte Strukturen aber begrenzt. Die Abgrenzung zwischen bukkaler und lingualer Defektlokalisation im Knochen ist nicht weiter möglich (Vandenberghe et al. 2008; Mohan et al. 2011; Kamburoğlu et al. 2013).

Die weiteren Ergebnisse zu den Untersuchungen der Knochendefekte unterschieden sich nicht aussagekräftig voneinander. Eine mögliche Erklärung für die nicht überlegene Betrachtungsweise im DVT kann die Anwesenheit von metallischen Körpern in der Aufnahme sein. Die Knochendefekte befinden sich in unmittelbarer Nähe zu den enossalen Implantaten. Eine Artefaktbildung um die Implantate kann die Auswertung negativ beeinflussen. Schwarz et al. evaluierten die Anwesenheit von periimplantären Knochendefekten zwar als einen ungenauen Verlauf des Implantat-Knochenübergangs, der krestale Knochen konnte laut dieser Untersuchung aber gut beurteilt werden (Schwarz et al. 2011). Andere Studien betrachteten in Anwesenheit von metallischen Körpern die Beurteilung des periimplantären Knochens in der DVT-Aufnahme als nicht zuverlässig (Corpas et al. 2011; Cremonini et al. 2011; Ritter et al. 2014).

In der aktuellen Studie musste nicht nur der besonders dichte krestale Knochen untersucht werden, sondern auch der locker strukturierte, spongiöse Knochen in Nachbarschaft zum Implantat. Daher können die Aufhärtungsartefakte in den DVT-Aufnahmen durchaus eine negative Auswirkung auf die Beurteilung gehabt haben (Benic et al. 2013). Das Auffinden der iatrogen gesetzten Implantatdefekte zeigte eine intermethodische Signifikanz. In den zweidimensionalen Zahnfilmaufnahmen ergab sich eine höhere Erkennungsrate der Defekte (konventioneller Zahnfilm $42 \%$, digitaler Zahnfilm $46 \%$ ) als in den DVT-Aufnahmen (10\%). Prozentual wurden die Defekte aber in allen Methoden gleichermaßen korrekt klassifiziert, so dass es hier keinen signifikanten Unterschied in den Ergebnissen gab. In den DVT-Aufnahmen wurde nur der Implantatdefekt C (Querrille 0,5 mm) korrekt zugeordnet. Der Defekt A (Längsrille in Windungstiefe) wurde nicht erkannt oder fehldiagnostiziert. Der Defekt B (1-2 Windungen entfernt) wurde in keiner der dreidimensionalen Aufnahmen erkannt. Eine mögliche Erklärung für die deutlich geringere Erkennungsrate der Implantatdefekte in den DVT-Aufnahmen ist das Vorliegen von Artefakten, hervorgerufen durch die Titanimplantate. Im Aufnahmeprozess durchdringen nur Röntgenstrahlen mit besonders hoher Intensität, d.h. 
mit besonders hoher Energie, die dichten Metallkörper. In direkter Nachbarschaft zum Implantat entsteht ein Effekt, der fälschlicherweise als fehlender Knochen interpretiert werden kann. Hierbei spricht man auch von einem missing data problem, ausgelöst durch den fehlerhaften Rekonstruktionsprozess (Schulze et al. 2010; Schulze et al. 2011). Die Aufhärtungsartefakte der Implantate könnten in dieser Studie dazu geführt haben, dass die iatrogenen Defekte an den Implantaten in der rekonstruierten DVT-Aufnahme zum Teil „überstrahlt" wurden und nur sehr schwer zu erkennen waren. Außerdem könnten fehlende Windungen beispielsweise von den Untersuchern fälschlicherweise als missing data problemArtefakte gedeutet worden sein. In den zweidimensionalen Aufnahmen der filmbasierten und digitalen Zahnfilme konnten weder Artefakte noch eine daraus folgende Beeinträchtigung der Bildauswertung durch die Untersuchergruppe festgestellt werden. In den DVT-Aufnahmen stellten die Untersucher in $70 \%$ der Datensätze Artefakte fest. $80 \%$ dieser Aufnahmen wurden durch die Artefakte negativ beeinflusst und erschwerten die Bildauswertung.

Die DVT-Technik wurde in vielen Studien als eine strahlenreduzierte und hochauflösende Alternative zur klassischen Computertomographie beschrieben (Ludlow und Ivanovic 2008). Die dreidimensionalen Aufnahmen zeigen eine gute Ortsauflösung und stellen die anatomischen Strukturen verzerrungs- und überlagerungsfrei dar (Hofmann et al. 2013). In Anwesenheit von metallischen Körpern kommt es jedoch zu einem Auftreten von Artefakten, ausgelöst durch die metallischen Körper im Strahlengang der Röntgenstrahlung. Dieser Effekt entsteht in derselben Weise wie in der Computertomographie, da die Rekonstruktionsalgorithmen beim CT und der DVT grundsätzlich dieselben sind (Schulze et al. 2010). Neuere DVT-Geräte bieten eine zusätzliche Metallartefaktreduktions-Funktion (MAR), die die Artefaktbildung in der Aufnahme reduzieren soll. Dabei handelt es sich fast ausschließlich um eine Modifikation des Rekonstruktionsalgorithmus, der die Artefaktbildung verringern soll (Zhang et al. 2007; Zhang et al. 2011). In der vorliegenden Untersuchung wurden alle Aufnahmen mit einer solchen Funktion durchgeführt. Die Beurteilung der Aufnahmen war laut der Untersucher dennoch negativ von Metallartefakten beeinflusst. Auch Kamburoğlu et al. kamen zu einem solchen Ergebnis. In der Untersuchung von Kamburoğlu wurden unter anderem periimplantäre Defekte mit und ohne MAR dargestellt. Dabei wurde in zwei verschiedenen DVT-Geräten eine bessere Bildgenauigkeit ohne die MAR-Funktion erzielt als mit einer Artefaktreduktionsfunktion (Kamburoğlu et al. 2013). Ursächlich für 
dieses Ergebnis könnte sein, dass es sich bei der MAR um eine Veränderung des Rekonstruktionsalgorithmus nach der Röntgenaufnahme handelt. Die eigentliche physikalische Ursache der Artefaktbildung beim Aufnahmeprozess des dreidimensionalen Bildes wird nicht verändert (Schulze et al. 2010).

Das Auffinden der Fremdmaterialien in den Schweinekieferpräparaten mit unterschiedlich hohen Röntgenopazitäten war im intermethodischen Vergleich nicht signifikant. Dennoch unterschieden sich die Ergebnisse der Klassifizierung der einzelnen Fremdmaterialien voneinander. Dabei ergaben die Ergebnisse der korrekten Defektzuordnung von Guttaperchastiften in den drei Verfahren einen signifikanten Unterschied. Die Unterschiede der Ergebnisse zur Defektzuordnung von B-TCP und der Stahlkugeln waren ohne Signifikanz im intermethodischen Vergleich. Die Stahlkugeln wurden von allen Fremdmaterialien am häufigsten erkannt und korrekt klassifiziert. Durch die hohe Dichte der Stahlkugeln wirken diese Körper in allen Aufnahmen sehr prominent und werden von den Untersuchern schnell erkannt. Die anderen Fremdmaterialien haben eine im Vergleich zum Implantat geringere Dichte und könnten daher häufiger übersehen werden. Eggers et al. kamen ebenfalls zu dem Schluss, dass Fremdkörper mit einer hohen Röntgenopazität einen größeren Kontrast zum umgebenden Gewebe produzieren. Ein geringer Kontrast zwischen schwach röntgenopaken Fremdkörpern und Gewebe bei gleichzeitiger geringer Größe der Fremdkörper führt zu einem deutlichen Unterschied in der Auffindbarkeit in einer dreidimensionalen Röntgenaufnahme (Eggers et al. 2007).

Die Durchmesserbestimmung der Stahlkugeln ergab im intermethodischen Vergleich einen signifikanten Unterschied. Ebenso unterschieden sich die Messabweichungen der zwei unterschiedlichen Durchmesser der Stahlkugeln signifikant voneinander. Die digitale Volumentomographie unterschied sich in den Mittelwerten der Kugeldurchmesser erheblich. Grund dafür können die schon zuvor diskutierten Aufhärtungsartefakte sein. Die Messergebnisse in den zweidimensionalen Methoden unterschieden sich zwar auch zwischen den unterschiedlichen Durchmessern, die Unterschiede waren dennoch nicht so erheblich wie bei der DVT. Da den Untersuchern die Herstellerangaben der Kugeln nicht bekannt waren, gingen Sie möglicherweise durch eine Erwartungshaltung davon aus, dass der Durchmesser der Kugeln in halben Millimeterschritten ansteigt. Dies würde das ungenauere Messergebnis für die 1,6 mm-Kugel erklären. Dies ist eine hypothetische Erklärung für die in allen Methoden 
kürzer ausfallenden Messergebnisse für die 1,6 mm-Stahlkugel. Die drei radiologischen Verfahren unterschieden sich in den Ergebnissen der Detektion der Guttaperchastifte signifikant voneinander. Die Ergebnisse ähnelten sich in den zweidimensionalen Aufnahmen in den korrekt klassifizierten, den fehldiagnostizierten und den nicht erkannten Guttaperchastiften. Die Ergebnisse in der digitalen Volumentomographie zeigten deutlich weniger korrekt zugeordnete Fremdmaterialien und eine wesentlich höhere Rate an nicht erkannten Guttaperchastiften.

Zusammenfassend ist auffällig, dass in Präparat 3 (Fremdmaterialien ß-TCP, 1xGuttapercha 30er Point, $1 \mathrm{~mm}$ Länge, Stahlkugel $1 \mathrm{~mm}$ ) der Guttaperchastift in keiner der Methoden erkannt wurde. Im Gegensatz zu den zwei weiteren Präparaten (Präparat 12 und 13), in denen Guttapercha als Fremdmaterial vorhanden ist, befindet sich in Präparat 3 zusätzlich eine Stahlkugel als Fremdkörper. Es ist möglich, dass durch die Anwesenheit der Stahlkugel die Aufmerksamkeit des Untersuchers auf die leicht zu erkennende Kugel konzentriert wird und somit ein Übersehen eines weniger röntgenopaken Fremdmaterials wahrscheinlicher wird. Des Weiteren ist in den zweidimensionalen Aufnahmen eine Überlagerung der beiden Fremdkörper möglich, die eine eingeschränkte Beurteilung der Aufnahme hinsichtlich einzelner Fremdmaterialien zur Folge hat. In der dreidimensionalen DVT-Aufnahme kann durch Aufhärtungsartefakte im Bereich der metallischen Körper das Nachbargewebe nicht eindeutig evaluiert werden. Daraus folgend kann ein Fremdkörper in direkter Nachbarschaft zu einer Stahlkugel in der DVT-Aufnahme überstrahlt sein und wird dadurch vom Untersucher übersehen. Die Detektion eines Fremdkörpers hängt sowohl von der Röntgenopazität ab als auch von seiner Größe und seiner Umgebung. Überlagerungen und Artefaktbildungen in der Umgebung können die Detektion beeinflussen. Folglich ist die Aufnahmetechnik ein weiterer Hauptfaktor zur Detektion eines Fremdkörpers (Eggers et al. 2007).

Die beta-Tricalciumphosphat-Keramik (ß-TCP) ist in dieser Untersuchung das Fremdmaterial mit der geringsten Dichte und einer geringen Röntgenopazität. Es ist Bestandteil der Präparate Nr. 3 und Nr. 12. Der prozentuale Anteil des korrekt identifizierten Materials in den beiden Präparaten war deutlich niedriger als der Anteil der korrekt erkannten Fremdmaterialen für die anderen Fremdmaterialien in dieser Untersuchung. In den zweidimensionalen Aufnahmen wurden $25 \%$ des inserierten B-TCP korrekt identifiziert. In den DVT-Aufnahmen waren es $10 \%$. Hauptsächlich wurde das Material nur in Präparat 12 detektiert. Nur ein Untersucher 
konnte das Fremdmaterial in einer konventionellen Zahnfilmaufnahme auch im Präparat Nr. 3 auffinden. Wie schon im Abschnitt zum Fremdmaterial Guttapercha diskutiert, kommen in Bezug auf das Präparat Nr. 3 mehrere mögliche Ursachen für dieses Ergebnis in Frage. Grundsätzlich ähnelt das ß-TCP aus konzeptionellen Gründen der Struktur von Alveolarknochen. Damit weist es bei einer Korngröße von 500-1000 $\mu \mathrm{m}$ eine dem trabekulären Knochen ähnliche Dichte und eine schwache Röntgenopazität auf. Daraus resultiert in Röntgenaufnahmen ein schwacher Kontrast zwischen dem Knochenersatzmaterial und dem Knochengewebe (Shalash et al. 2013). Dies könnte die Ursache sein, dass das B-TCP seltener als die anderen Fremdmaterialen in den Aufnahmen detektiert wurde. Ein Vergleich zwischen den konventionellen und den digitalen Zahnfilmaufnahmen ergab in dieser Untersuchung keinen signifikanten Unterschied. Die Untersuchergruppe befundete die Aufnahmen in sehr ähnlicher und zum Teil in identischer Weise. Wie schon im oben stehenden Abschnitt zu den metrischen Messwerten der Implantate diskutiert, ergab sich in Bezug auf die Messabweichungen der Implantatlängen ein signifikanter Unterschied, der jedoch eher durch die Rahmenbedingungen dieser Studie begründet ist. In vergangenen Studien konnte zwischen konventionellen und digitalen Zahnfilmaufnahmen kein deutlicher Unterschied in der Diagnostik von periapikalen, pathologischen Veränderungen festgestellt werden. Vorteil der digitalen Zahnfilmaufnahme ist die reduzierte Strahlenbelastung (Tirrell et al. 1996; Barbat und Messer 1998; Sullivan et al. 2000; Stavropoulos und Wenzel 2007). Im Vergleich der zweidimensionalen Methoden gegenüber der dreidimensionalen DVT wurde deutlich, dass in dieser Untersuchung die Alveolardefekte, Implantatdefekte und die Fremdmaterialien häufiger in den zweidimensionalen Röntgenmethoden korrekt erkannt wurden. In den DVTAufnahmen wurden weniger Defekte bzw. Fremdmaterialien erkannt. Die Unversehrtheit des Gewebes - in Abwesenheit von Defekten und Fremdmaterialien - wurde mit allen Methoden ähnlich gut evaluiert. Die zweidimensionalen Verfahren zeigten somit gute Ergebnisse bei Vorliegen und in Abwesenheit der Defekte und Fremdmaterialien. In den DVT-Aufnahmen hingegen konnte nur die Abwesenheit von Defekten und Fremdkörpern relativ sicher beurteilt werden. Zum einen kann die Artefaktbildung in den DVT-Bilddaten ursächlich für dieses Ergebnis sein, zum anderen ist möglicherweise auch die geringe Erfahrung der Untersucher mit der Befundung von DVT-Aufnahmen ein erklärbarer Grund für viele nicht erkannte Defekte und Fremdmaterialien. Die Untersucher hatten alle den gleichen Wissensstand in der Befundung von konventionellen und digitalen Zahnfilmaufnahmen sowie von DVT- 
Aufnahmen. Die Erfahrung im Umgang mit zweidimensionalen Aufnahmen war jedoch größer. Innerhalb der Untersuchergruppe ergab sich eine signifikante Abweichung für die Messungen der Implantatdurchmesser. Diese Abweichung beschränkt sich auf einen Untersucher. Die Ergebnisse der Messabweichungen der Implantatlängen sowie die Beurteilung der Alveolar- und Implantatdefekte sowie der Fremdmaterialien zeigten keine signifikanten Unterschiede innerhalb der Untersuchergruppe. Die basalen Kenntnisse der Probanden in der Auswertung von DVT-Aufnahmen könnten ursächlich für das geringe Auffinden von Defekten und Fremdmaterialien in den dreidimensionalen Aufnahmen sein. Im Vergleich innerhalb der Untersuchergruppe ergaben sich eine unterschiedliche Präzision und Streuung bei den Auswertungen. Gegebenenfalls könnte eine Vorauswahl der Untersucher anhand einer Probeevaluation die Streuung der Ergebnisse reduzieren.

Die Implantologie ist zu einem festen Bestandteil der Zahnmedizin geworden und wird zukünftig weiter an Stellenwert gewinnen. Implantate stellen häufig die Lösung prothetischer Versorgungsproblematiken dar. In Hinblick auf diese Entwicklung werden diagnostische Maßnahmen zur Planung der Implantatinsertion sowie navigationsgestützte Implantation ebenfalls wachsen. Die dreidimensionale Bildgebung wird die etablierte zweidimensionale Bildgebung nicht ablösen. Jedoch wird mit einer komplexen Behandlungssituation die Anwendung der dreidimensionalen Diagnostik zunehmen, um eine überlagerungsfreie Darstellung der angrenzenden anatomischen Strukturen zu gewährleisten. Zur besseren Darstellung von bereits inserierten Implantaten sollte die DVT-Technik speziell in Bezug auf metallinduzierte Artefakte weiter entwickelt werden. In dieser Studie wurde gezeigt, dass trotz einer MAR-Aufnahmefunktion die vollständige Beurteilung von metallischen Körpern und deren direkt angrenzenden Nachbarstrukturen mit dem hier verwendeten Röntgengerät nicht zweifelsfrei möglich ist. 


\section{Schlussfolgerung}

- Alle drei evaluierten Verfahren zeigten eine vergleichbare Darstellungsqualität der untersuchten Strukturen.

- Trotz einer MAR-Funktion treten in Anwesenheit von Metallkörpern in der DVTAufnahme Artefakte auf, die die Bildauswertung negativ beeinflussen.

- In dieser Studie wurden die untersuchten Strukturen und deren Defekte häufiger in den zweidimensionalen Zahnfilmverfahren korrekt beurteilt als in der dreidimensionalen DVT-Technik. 


\section{Zusammenfassung}

Ziel der Studie: Ziel war es, die Darstellbarkeit von dentalen Implantaten sowie des umliegenden Gewebes mit iatrogenen Defekten und Fremdmaterialien in drei Röntgenverfahren zu vergleichen. Anschließend an eine vorangegangene Studie (Jarnot 2011) evaluierten in der gegenwärtigen Studie zehn Probanden mit gleichem Wissens- und Ausbildungstand zahnärztliche Röntgenaufnahmen unter dem Aspekt der Vergleichbarkeit von zwei- und dreidimensionalen Verfahren. Diese Untersuchung wurde anhand des konventionellen und digitalen Zahnfilmverfahrens und der dreidimensionalen digitalen Volumentomographie durchgeführt. Die Studie bearbeitet die Hypothese, dass die dreidimensionale digitale Volumentomographie exaktere und bessere Informationen über eine Implantat-Knochensituation liefert als konventionelle und digitale zweidimensionale Zahnfilmaufnahmen.

Methodik: Zehn Schweinekieferpräparate, versehen mit unterschiedlichen Implantaten, Gewebe- und Implantatdefekten sowie inserierten Fremdmaterialien wurden radiologisch dargestellt. Eine Probandengruppe mit gleichem Wissens- und Ausbildungsstand evaluierte die Aufnahmen in randomisierter Abfolge anhand eines Prüfbogens.

Ergebnisse: Alle untersuchten Verfahren zeigten eine vergleichbare Darstellungsqualität der untersuchten Strukturen. Signifikante Unterschiede ergaben sich in den Ergebnissen der Evaluation der Implantatdefekte und des Fremdmaterials Guttapercha. Die Ergebnisse der evaluierten DVT-Aufnahmen zeigten diesbezüglich deutlich weniger detektierte Defekte und Fremdmaterialien. Ein lingualer Perforationsdefekt des Alveolarknochens konnte von den Probanden jedoch nur in den DVT-Aufnahmen korrekt klassifiziert werden.

Schlussfolgerung: Die dreidimensionale DVT ermöglichte den Untersuchern in dieser Studie trotz der überlagerungsfreien Darstellung des Aufnahmevolumens keine signifikant bessere Beurteilung im Vergleich zu den zweidimensionalen Röntgenverfahren. Erklärbar ist dieses Ergebnis einerseits durch die Metallartefakte in den DVT-Aufnahmen, andererseits durch die basalen DVT-Kenntnisse der Zahnmedizinstudenten als Untersucher dieser Studie. Zusammenfassend ist festzustellen, dass der Zahnfilm, ob konventionell oder digital, im klinischen Alltag eine wichtige Rolle in der Zahnheilkunde einnimmt und auch in der 
implantologischen Diagnostik eine exakte und strahlenarme Methode zur Beurteilung des periimplantären Gewebes um ein inseriertes Implantat darstellt. 


\section{Anhang}

\section{Tabellenverzeichnis}

Tabelle 1: Zuordnung der Implantatdefekte, Alveolardefekte und Fremdkörper zu den Präparaten... 28

Tabelle 2: Technische Daten des Heliodent MD (Sirona Dental Systems, Bensheim, Deutschland).. 30

Tabelle 3: Technische Daten des Miniray, SOREDEX, Helsinki, Finnland ................................... 32

Tabelle 4: Technische Daten des PaX-Zenith 3D, Orangedental-Vatech, Südkorea ......................... 34

Tabelle 5: Aufnahmeparameter des DVT-Gerätes PaX-Zenith 3D ......................................... 35

Tabelle 6: Mittelwerte der Messabweichung der Implantatlängen. ............................................... 52

Tabelle 7: Mittelwerte der Messabweichung der Implantatdurchmesser ........................................ 55

-Toc443928800

Tabelle 9: Messabweichungen für den Stahlkugeldurchmesser $\emptyset 1,6 \mathrm{~mm}$ 71

\section{Abbildungsverzeichnis}

Abbildung 1: Versuchsaufbau zur Aufnahme eines konventionellen Zahnfilmes.. ......................... 30

Abbildung 2: Versuchsaufbaus zur Aufnahme eines digitalen Zahnfilmes. .................................. 33

Abbildung 3: Versuchsaufbau DVT (PaX-Zenith 3D, Orangedental-Vatech, Südkorea) .................... 36

Abbildung 4: Prüfbogen zur Evaluation der Bilddaten ........................................................ 410

Abbildung 5: Differenzierung der Nachbargewebe im konv. ZF und dig. ZF ............................. 44

Abbildung 6: Differenzierung der Nachbargewebe in Ausschnitten des DVT .............................. 45

Abbildung 7: Knochendefekte in den zweidimensionalen Röntgenaufnahmen .............................. 45

Abbildung 8: Implantatdefekt in den zweidimensionalen Röntgenaufnahmen .............................. 46

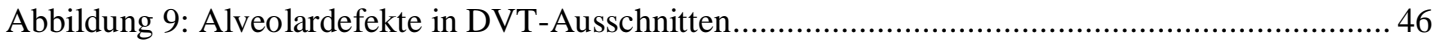

Abbildung 10: Implantatdefekte in DVT-Ausschnitten ........................................................ 47

Abbildung 11: Fremdmaterialien in den zweidimensionalen Zahnfilmaufnahmen .............................. 47

Abbildung 12: Fremdmaterialien in DVT-Ausschnitten ..................................................... 48

Abbildung 13: Messung der Implatatlänge und des Implantatdurchmessers im dig. ZF .................. 48

Abbildung 14: Messung der Implatatlänge und des Implantatdurchmessers in DVT-Ausschnitten .... 49

Abbildung 15: Absolute Messabweichung der Implantatlängen in konv. ZF, dig. ZF u. DVT .......... 51

Abbildung 16: Darstellung der korrekt gemessenen Implantatlängen im konv. ZF, dig. ZF u. DVT .. 52

Abbildung 17: Absolute Messabweichungen der Implantatlängen im konv. ZF, dig. ZF u. DVT..... 53

Abbildung 18: Messabweichungen der Implantatdurchmesser im konv. ZF, dig.ZF u. DVT............ 54

Abbildung 19: Darstellung der korrekt gemessenen Implantatdurchmesser im konv. ZF, dig. ZF u.

DVT.

Abbildung 20: Messabweichungen der Implantatdurchmesser in den drei radiologischen Methoden. 56

Abbildung 21: Darstellung von erkannten Alveolardefekten, unterteilt in korrekt und falsch

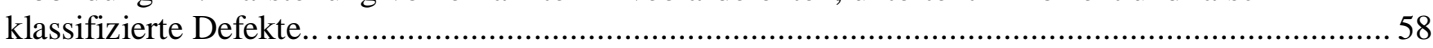

Abbildung 22: Alveolardefekt A in Präparat 2 im konv. ZF, dig. ZF und DVT ............................. 58

Abbildung 23: Alveolardefekt B in Präparat 12 im konv. ZF, dig. ZF und DVT .......................... 59

Abbildung 24: Alveolardefekt „Bonesplitting“ in Präparat 10 im konv. ZF, dig. ZF und DVT ......... 60

Abbildung 25: Alveolardefekt „Überdimension“ im Präparat 13 im konv. ZF, dig. ZF und DVT ...... 61

Abbildung 26: Alveolardefekt „Ersatz, lingual perforiert“ in Präparat 14 im konv. ZF, dig. ZF und

DVT.

Abbildung 27: Darstellung der erkannten Implantatdefekte, unterteilt in korrekt und falsch

klassifizierte Defekte.

Abbildung 28: Implantatdefekt A in Präparat 5 im konv. ZF, dig. ZF und DVT 
Abbildung 29: Implantatdefekt B in Präparat 3 im konv. ZF, dig. ZF und DVT

Abbildung 30: Implantatdefekt $\mathrm{C}$ in Präparat 8 im konv. ZF, dig. ZF und DVT ........

Abbildung 31: Implantatdefekt $\mathrm{C}$ in Präparat $12 \mathrm{im} \mathrm{konv.} \mathrm{ZF,} \mathrm{dig.} \mathrm{ZF} \mathrm{und} \mathrm{DVT}$

Abbildung 32: Darstellung der erkannten Fremdkörper, unterteilt in korrekt und falsch

klassifizierte Fremdkörper.

Abbildung 33: Häufigkeit der korrekten Fremdkörperklassifizierung in den drei Röntgenmethoden. 67

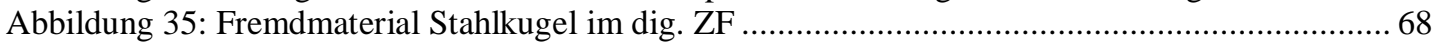

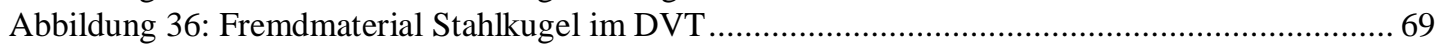

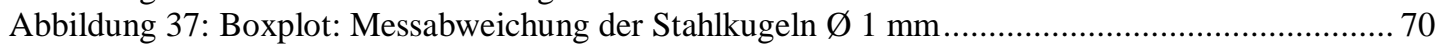

Abbildung 38: Boxplot: Messabweichung der Stahlkugeln $\varnothing$ 1,6 mm .................................... 71

Abbildung 39: Fremdmaterial Guttaperchastifte im konv. ZF ............................................... 72

Abbildung 40: Fremdmaterial Guttaperchastifte im dig. ZF .................................................. 73

Abbildung 41: Fremdmaterial Guttaperchastifte im DVT ...................................................... 73

Abbildung 42: Darstellung der korrekt klassifizierten, nicht erkannten und falsch diagnostizierten

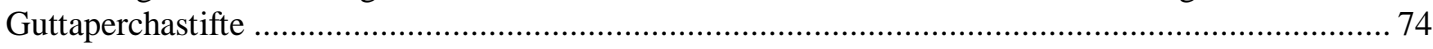

Abbildung 43: Darstellung des korrekt klassifizierten und nicht erkannten ß-TCP ........................ 75

Abbildung 44: Fremdmaterial beta-Tricalciumphosphat (Präparat 3) im konv. ZF, dig. ZF

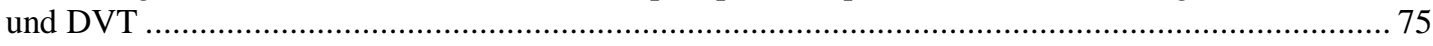

Abbildung 45: Fremdmaterial beta-Tricalciumphosphat (Präparat 12) im konv. ZF, dig. ZF

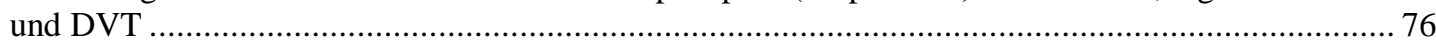

Abbildung 46: Beispiele für Bildartefakte im Bereich der metallischen Körper in DVT-Aufnahmen 77

Abbildung 47: Vorhandene Knochendefekte: Vergleich der zweidimensionalen Verfahren mit der

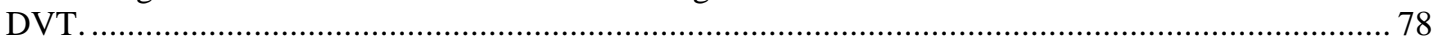

Abbildung 48: Nicht vorhandene Knochendefekte: Vergleich der zweidimensionalen Verfahren mit der DVT..

Abbildung 49: Implantatdefekt vorhanden: Vergleich der zweidimensionalen Verfahren mit der DVT..

Abbildung 50: Kein Implantatdefekt: Vergleich der zweidimensionalen Verfahren mit der DVT....... 80 Abbildung 51: Fremdkörper vorhanden: Vergleich der zweidimensionalen Verfahren mit der DVT..81 Abbildung 52: Kein Fremdkörper vorhanden: Vergleich der zweidimensionalen Verfahren mit der DVT.

Abbildung 53: Vergleich der Untersucher bezüglich der Messpräzision für die Implantatdurchmesser..

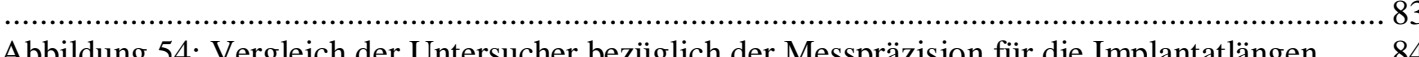

Abbildung 54: Vergleich der Untersucher bezüglich der Messpräzision für die Implantatlängen....... 84 Abbildung 55: Alveolardefekte: Vergleich der Untersucher bezüglich der korrekten Klassifizierung

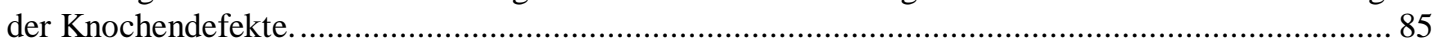
Abbildung 56: Implantatdefekte: Vergleich der Untersucher bezüglich der korrekten Klassifizierung

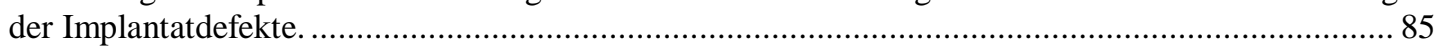
Abbildung 57: Fremdmaterialien: Vergleich der Untersucher bezüglich der korrekten Klassifizierung

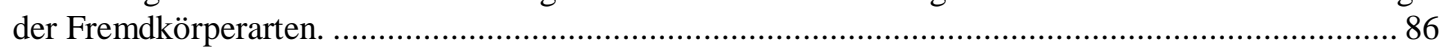




\section{Literaturverzeichnis}

Abraham CM (2014): A brief historical perspective on dental implants, their surface coatings and treatments. Open Dent $\mathrm{J} \underline{8}, 50-55$

Albrektsson T, Brånemark PI, Hansson HA, Lindström J (1981): Osseointegrated Titanium Implants: Requirements for Ensuring a Long-Lasting, Direct Bone-to-Implant Anchorage in Man. Acta Orthop Scand. 52, 155-170

Al-Ekrish AA, Ekram M (2011): A comparative study of the accuracy and reliability of multidetector computed tomography and cone beam computed tomography in the assessment of dental implant site dimensions. Dentomaxillofac Radiol $\underline{40}$, 67-75

Barbat J, Messer HH (1998): Detectability of artificial periapical lesions using direct digital and conventional radiography. J Endod 24, 837-842

Benic GI, Sancho-Puchades M, Jung RE, Deyhle H, Hämmerle CHF (2013): In vitro assessment of artifacts induced by titanium dental implants in cone beam computed tomography. Clin Oral Implants Res 24, 378-383

Binon PP (2000): Implants and components: entering the new millennium. Int J Oral Maxillofac Implants 15, 76-94

Böhm B: Erste Untersuchungen zum CASCADE-Röntgendetektor und Messung zur Neutron-Spiegelneutron-Oszillation. Phys.-Math. Diss. Heidelberg 2007

Braut V, Bornstein MM, Belser U, Buser D (2011): Thickness of the anterior maxillary facial bone wall-a retrospective radiographic study using cone beam computed tomography. Int J Periodontics Restorative Dent 31, 125-131

Brunski JB, Puleo DA, Nanci A (2000): Biomaterials and biomechanics of oral and maxillofacial implants: current status and future developments. Int J Oral Maxillofac Implants $\underline{15}, 15-46$

Buser D, Weber HP, Bragger U, Balsiger C (1991): Tissue integration of one-stage ITI implants: 3-year results of a longitudinal study with Hollow-Cylinder and Hollow-Screw implants. Int J Oral Maxillofac Implants $\underline{6}, 405-412$

Chan H, Misch K, Wang H (2010): Dental Imaging in Implant Treatment Planning. Implant Dentistry $\underline{19}, 288-298$

Chen ST, Wilson TG, Hämmerle CH (2004): Immediate or early placement of implants following tooth extraction: review of biologic basis, clinical procedures, and outcomes. Int $\mathrm{J}$ Oral Maxillofac Implants $\underline{19}, 12-25$

Cohenca N, Simon JH, Roges R, Morag Y, Malfaz JM (2007): Clinical indications for digital imaging in dento-alveolar trauma. Part 1: traumatic injuries. Dent Traumatol 23, 95-104

Corpas Ldos S, Jacobs R, Quirynen M, Huang Y, Naert I, Duyck J (2011): Peri-implant bone tissue assessment by comparing the outcome of intra-oral radiograph and cone beam computed tomography analyses to the histological standard. Clin Oral Impl Res $\underline{22}, 492-499$ 
Cremonini C, Dumas M, Pannuti C, Neto J, Cavalcanti M, Lima L (2011): Assessment of linear measurements of bone for implant sites in the presence of metallic artefacts using cone beam computed tomography and multislice computed tomography. Int J Oral Maxillofac Surg $\underline{40}, 845-850$

Damstra J, Fourie Z, Huddleston Slater JJR, Ren Y (2010): Accuracy of linear measurements from cone-beam computed tomography-derived surface models of different voxel sizes. Am J Orthod Dentofacial Orthop 137, 16.e1-6; discussion 16-7

DelBalso AM, Greiner FG, Licata M (1994): Role of diagnostic imaging in evaluation of the dental implant patient. Radiographics 14, 699-719

Eggers G, Welzel T, Mukhamadiev D, Wörtche R, Hassfeld S, Mühling J (2007): X-rayBased Volumetric Imaging of Foreign Bodies: A Comparison of Computed Tomography and Digital Volume Tomography. J Oral Maxillofac Surg ㅌ5, 1880-1885

Estrela C, Bueno MR, Azevedo BC, Azevedo JR, Pécora JD (2008): A new periapical index based on cone beam computed tomography. J Endod $\underline{34}$, 1325-1331

European Commission of Energy (2004): Radiation protection 136. European guidelines on radiation protection in dental radiology. Office for Official Publications of the European Communities https://ec.europa.eu/energy/sites/ener/files/documents/136.pdf [Zugriff am 26.1.2015]

European Commission of (2012): Radiation Protection 172. Cone Beam CT for Dental and Maxillofacial Radiology - Evidence-based guidelines. EnergyOffice for Official Publications of the European Communities http://www.sedentexct.eu/files/radiation_protection_172.pdf [Zugriff am 1.12.2014]

Garg AK, Vicari A (1995): Radiographic modalities for diagnosis and treatment planning in implant dentistry. Implant Soc $\underline{5}, 7-11$

Haghanifar S, Moudi E, Mesgarani A, Bijani A, Abbaszadeh N (2014): A comparative study of cone-beam computed tomography and digital periapical radiography in detecting mandibular molars root perforations. Imaging Sci Dent 44, $115-119$

Hämmerle CH, Chen ST, Wilson TG Jr (2004): Consensus statements and recommended clinical procedures regarding the placement of implants in extraction sockets. Int J Oral Maxillofac Implants $\underline{19}, 26-28$

Harris D, Horner K, Gröndahl K, Jacobs R, Helmrot E, Benic GI, Bornstein MM, Dawood A, Quirynen M (2012): E.A.O. guidelines for the use of diagnostic imaging in implant dentistry 2011. A consensus workshop organized by the European Association for Osseointegration at the Medical University of Warsaw. Clin Oral Impl Res 23, 1243-1253

Hassan B, Couto Souza P, Jacobs R, Azambuja Berti S, Stelt P (2010): Influence of scanning and reconstruction parameters on quality of three-dimensional surface models of the dental arches from cone beam computed tomography. Clin Oral Invest 14, 303-310

Hiasa K, Abe Y, Okazaki Y, Nogami K, Mizumachi W, Akagawa Y (2011): Preoperative Computed Tomography-Derived Bone Densities in Hounsfield Units at Implant Sites Acquired Primary Stability. ISRN Dentistry 2011, 1-5 
Hofmann E, Schmid M, Sedlmair M, Banckwitz R, Hirschfelder U, Lell M (2013): Comparative study of image quality and radiation dose of cone beam and low-dose multislice computed tomography - an in-vitro investigation. Clin Oral Invest $\underline{18}, 301-311$

Howerton WB Jr, Mora MA (2008): Advancements in Digital Imaging: What is New and on the Horizon? JADA (The Journal of the American Dental Association) 139, 20S-24S

Ibrahim N, Parsa A, Hassan B, van der Stelt P, Wismeijer D (2013): Diagnostic imaging of trabecular bone microstructure for oral implants: a literature review. Dentomaxillofac Radiol $\underline{42}, 1-5$

Inokoshi M, Kanazawa M, Minakuchi S (2012): Evaluation of a complete denture trial method applying rapid prototyping. Dent Mater J $\underline{31}, 40-46$

Jarnot L: Darstellbarkeit von Knochenstrukturen und enossalen Implantaten mit der Flächendetektor-Volumencomputertomographie und mit konventionellen radiologischen Verfahren. Med. Diss. Göttingen 2011

Jivraj S, Chee W (2006): Rationale for dental implants. Br Dent J 200, 661-665

Jokstad A, Braegger U, Brunski JB, Carr AB, Naert I, Wennerberg A (2003): Quality of dental implants. Int Dent J $\underline{53}, 409-443$

Kamburoğlu K, Kolsuz E, Murat S, Eren H, Yüksel S, Paksoy CS (2013): Assessment of buccal marginal alveolar peri-implant and periodontal defects using a cone beam CT system with and without the application of metal artefact reduction mode. Dentomaxillofac Radiol $\underline{42}, 1-9$

Klokkevold PR, Newman MG (2000): Current status of dental implants: a periodontal perspective. Int J Oral Maxillofac Implants $\underline{15}, 56-65$

Kobayashi K, Shimoda S, Nakagawa Y, Yamamoto A (2004): Accuracy in measurement of distance using limited cone-beam computerized tomography. Int J Oral Maxillofac Implants $\underline{19}, 228-231$

Krause J: Untersuchungen zum erforderlichen Field of View für die bildgebende 3DDiagnostik in der Zahnmedizin. Med. Diss. Berlin 2013

Krzyżostaniak J, Surdacka A, Kulczyk T, Dyszkiewicz-Konwińska M, Owecka M (2014):

Diagnostic accuracy of cone beam computed tomography compared with intraoral radiography for the detection of noncavitated occlusal carious lesions. Caries Res $\underline{48}, 461-$ 466

Lindner M: Entwicklung eines zählenden Pixeldetektors für "Digitales Röntgen". Nat.-Wiss. Diss. Bonn 2001

Loubele M, Jacobs R, Maes F, Denis K, White S, Coudyzer W, Lambrichts I, van Steenberghe D, Suetens P (2008): Image quality vs radiation dose of four cone beam computed tomography scanners. Dentomaxillofac Radiol 37, 309-319

Loubele M, Bogaerts R, van Dijck E, Pauwels R, Vanheusden S, Suetens P, Marchal G, Sanderink G, Jacobs R (2009): Comparison between effective radiation dose of CBCT and MSCT scanners for dentomaxillofacial applications. Eu J Radiol 71, 461-468 
Low KM, Dula K, Bürgin W, von Arx T (2008): Comparison of Periapical Radiography and Limited Cone-Beam Tomography in Posterior Maxillary Teeth Referred for Apical Surgery. J Endod 34, 557-562

Ludlow JB, Ivanovic M (2008): Comparitive dosimetry of dental CBCT devices and 64-slice CT for oral and maxillofacial radiology. Oral Surg Oral Med Oral Pathol Oral Radiol Endod $\underline{106}, 106-114$

Ludlow JB, Timothy R, Walker C, Hunter R, Benavides E, Samuelson DB, Scheske MJ (2015): Effective dose of dental CBCT-a meta analysis of published data and additional data for nine CBCT units. Dentomaxillofac Radiol 44, 1-25

Matherne RP, Angelopoulos C, Kulild JC, Tira D (2008): Use of cone-beam computed tomography to identify root canal systems in vitro. J Endod $\underline{34}, 87-89$

Medelnik J, Hertrich K, Steinhäuser-Andresen S, Hirschfelder U, Hofmann E (2011): Accuracy of anatomical landmark identification using different CBCT- and MSCT-based 3D images. J Orofac Orthop $\underline{72}$, 261-278

Mohan R, Singh A, Gundappa M (2011): Three-dimensional imaging in periodontal diagnosis - Utilization of cone beam computed tomography. J Indian Soc Periodontol $\underline{15}$, $11-17$

Mupparapu M, Singer SR (2004): Implant Imaging for the Dentist. J Can Dent Assoc 70, 32

Nabha W, Hong Y, Cho J, Hwang H (2014): Assessment of metal artifacts in threedimensional dental surface models derived by cone-beam computed tomography. Korean $\mathbf{J}$ Orthod 44,229

Nackaerts O, Maes F, Yan H, Couto Souza P, Pauwels R, Jacobs R (2011): Analysis of intensity variability in multislice and cone beam computed tomography. Clin Oral Implants Res $\underline{22}, 873-879$

Neugebauer J, Ritter L, Mischkowski R, Zöller JE (2006): Three-dimensional diagnostics, planning and implementation in implantology. Int J Comput Dent $\underline{9}, 307-319$

Nitsche T, Menzebach M, Wiltfang J (2012): S2-k-Leitlinie: Leitlinie Indikationen zur implantologischen 3D-Röntgendiagnostik und navigationsgestürtzten Implantologie. Deutsche Gesellschaft für Zahn-,Mund- und Kieferheilkunde. http://www.dgzmk.de/uploads/tx_szdgzmkdocuments/20120508_Leitlinie_navigierte_Impla ntatinsertion.pdf [Zugriff am 5.3.2013]

Parithimarkalaignan S, Padmanabhan TV (2013): Osseointegration: An Update. J Indian Prosthodont Soc 13, 2-6

Patel S (2009): New dimensions in endodontic imaging: Part 2. Cone beam computed tomography. Int Endod J 42, 463-475

Patel S, Dawood A, Whaites E, Pitt Ford T (2009a): New dimensions in endodontic imaging: part 1. Conventional and alternative radiographic systems. Int Endod J 42, 447-462

Patel S, Dawood A, Wilson R, Horner K, Mannocci F (2009b): The detection and management of root resorption lesions using intraoral radiography and cone beam computed tomography - an in vivo investigation. Int Endod J $\underline{42}, 831-838$ 
Poeschl PW, Schmidt N, Guevara-Rojas G, Seemann R, Ewers R, Zipko HT, Schicho K (2013): Comparison of cone-beam and conventional multislice computed tomography for image-guided dental implant planning. Clin Oral Invest 17, 317-324

Raghav N, Reddy SS, Giridhar A, Murthy S, Yashodha Devi B, Santana N, Rakesh N, Kaushik A (2010): Comparison of the efficacy of conventional radiography, digital radiography, and ultrasound in diagnosing periapical lesions. Oral Surg Oral Med Oral Pathol Oral Radiol Endod 110, 379-385

Ritter L, Elger MC, Rothamel D, Fienitz T, Zinser M, Schwarz F, Zöller JE (2014): Accuracy of peri-implant bone evaluation using cone beam CT, digital intra-oral radiographs and histology. Dentomaxillofac Radiol, 20130088

Rodrigo D, Aracil L, Martin C, Sanz M (2010): Diagnosis of implant stability and its impact on implant survival: a prospective case series study. Clin Oral Impl Res 21, 255-261

Scarfe WC (2006): Clinical Applications of Cone-Beam Computed Tomography in Dental Practice. J Can Dent Assoc $\underline{72}, 75-80$

Scarfe WC, Czerniejewski VJ, Farman AG, Avant SL, Molteni R (1999): In vivo accuracy and reliability of color-coded image enhancements for the assessment of periradicular lesion dimensions. Oral Surg Oral Med Oral Pathol Oral Radiol Endod 88, 603-611

Scarfe WC, Levin MD, Gane D, Farman AG (2009): Use of Cone Beam Computed Tomography in Endodontics. Int J Dent 2009, 1-20

Schliephake H, Rödiger M, Phillips K, McGlumphy EA, Chacon GE, Larsen P (2012): Early loading of surface modified implants in the posterior mandible - 5 year results of an open prospective non-controlled study. J Clin Periodontol $\underline{39}, 188-195$

Schulze R, Haßfeld S, Schulze D (2009): S1 Empfehlung: Dentale Volumentomographie (DVT). Deutsche Gesellschaft für Zahn-, Mund- und Kieferheilkunde

http://www.ag-kiefer.de/uploads/media/S1 Empfehlung Dentale Volumentomographie.pdf [Zugriff am 14.6.2013]

Schulze R, Berndt D, D'Hoedt B (2010): On cone-beam computed tomography artifacts induced by titanium implants. Clin Oral Impl Res 21, 100-107

Schulze R, Heil U, Gross D, Bruellmann D, Dranischnikow E, Schwanecke U, Schoemer E (2011): Artefacts in CBCT: a review. Dentomaxillofac Radiol 40, 265-273

Schulze R, Deppe H, Betz W, Maager B, Beuer F, Ritter L, Bargholz C, Ahlers O, Terhyden H, Hirsch C et al. (2013): S2-k-Leitlinie: Dentale digitale Volumentomographie. Deutsche Gesellschaft für Zahn-, Mund- und Kieferheilkunde http://www.dgzmk.de/uploads/tx_szdgzmkdocuments/0830051_S2k_Dentale_Volumentomographie_2013-10.pdf [Zugriff am 20.1.2014]

Schwarz F, Sahm N, Mihatovic I, Golubovic V, Becker J (2011): Surgical therapy of advanced ligature-induced peri-implantitis defects: cone-beam computed tomographic and histological analysis. J Clin Periodontol $\underline{38}$, 939-949 
Sennerby L, Meredith N (2008): Implant stability measurements using resonance frequency analysis: biological and biomechanical aspects and clinical implications. Periodontol 2000 47, 51-66

Shalash MA, Rahman HA, Azim AA, Neemat AH, Hawary HE, Nasry SA (2013): Evaluation of horizontal ridge augmentation using beta tricalcium phosphate and demineralized bone matrix: A comparative study. J Clin Exp Dent, e253-9

Sitzmann F, Benz C (2000): Wissenschaftliche Stellungnahme: Digitale Radiographie. Deutsche Gesellschaft für Zahn-, Mund- und Kieferheilkunde http://www.dgzmk.de/uploads/tx_szdgzmkdocuments/Digitale_Radiographie.pdf

[Zugriff am 15.4.2013]

Stavropoulos A, Wenzel A (2007): Accuracy of cone beam dental CT, intraoral digital and conventional film radiography for the detection of periapical lesions. An ex vivo study in pig jaws. Clin Oral Investig 11, 101-106

Sullivan JE, Di Fiore PM, Koerber A (2000): Radiovisiography in the detection of periapical lesions. J Endod 26, 32-35

Suomalainen A, Vehmas T, Kortesniemi M, Robinson S, Peltola J (2008): Accuracy of linear measurements using dental cone beam and conventional multislice computed tomography. Dentomaxillofac Radiol 37, 10-17

Tirrell BC, Miles DA, Brown CE, Legan JJ (1996): Interpretation of chemically created lesions using direct digital imaging. J Endod 22, 74-78

Valizadeh et al. (2012): Evaluation of Cone Beam Computed Tomography (CBCT) system: comparison with intraoral periapical radiography in proximal caries detection. J Dent Res Dent Clin Dent Prospect $\underline{6}, 1-5$

Van Assche N, van Steenberghe D, Guerrero ME, Hirsch E, Schutyser F, Quirynen M, Jacobs R (2007): Accuracy of implant placement based on pre-surgical planning of threedimensional cone-beam images: a pilot study. J Clin Periodontol $\underline{34}, 816-821$

Van der Stelt PF (2005): Filmless imaging: The uses of digital radiography in dental practice. JADA (The Journal of the American Dental Association) 136, 1379-1387

Van der Stelt PF (2008): Better Imaging: The Advantages of Digital Radiography. J Am Dent Assoc 139, 7S-13S

Vandenberghe B, Jacobs R, Yang J (2007): Diagnostic validity (or acuity) of 2D CCD versus 3D CBCT-images for assessing periodontal breakdown. Oral Surg Oral Med Oral Pathol Oral Radiol Endod 104, 395-401

Vandenberghe B, Jacobs R, Yang J (2008): Detection of periodontal bone loss using digital intraoral and cone beam computed tomography images: an in vitro assessment of bony and/or infrabony defects. Dentomaxillofac Radiol $\underline{37}$, 252-260

Vazquez L, Saulacic N, Belser U, Bernard J (2008): Efficacy of panoramic radiographs in the preoperative planning of posterior mandibular implants: a prospective clinical study of 1527 consecutively treated patients. Clin Oral Implants Res $\underline{19}, 81-85$ 
Zhang X, Wang J, Xing L (2011): Metal artifact reduction in X-ray computed tomography (CT) by constrained optimization. Med Phys $\underline{38}, 701-711$

Zhang Y, Zhang L, Zhu XR, Lee AK, Chambers M, Dong L (2007): Reducing metal artifacts in cone-beam CT images by preprocessing projection data. Int J Radiat Oncol Biol Physbuser. 67, 924-932 


\section{Danksagung}

Mein besonderer Dank gilt Frau PD Dr. med. dent. Sabine Sennhenn-Kirchner für das Überlassen des Dissertationsthemas und die herausragende wissenschaftliche Betreuung der Arbeit. Ihre vielfältigen fachlichen Anregungen, ständige Motivation und konstruktive Kritik haben mich während der Arbeit an meiner Dissertation stets unterstützt. Herzlichen Dank für die enge und engagierte Begleitung.

Ich bedanke mich ebenso bei Herrn Dr. med dent. Stephan Klotz, Göttingen, für das Bereitstellen des digitalen Röntgengerätes in seiner Praxis sowie der Praxisgemeinschaft Dr. med. dent. Nensa, Bremen, für das Digitalisieren der konventionellen Zahnfilmaufnahmen. 
